\title{
DESCRIPTION OF CLAY ANISOTROPY EMPLOYING THE CONCEPT OF DIRECTIONAL VARIATION OF FOROSITY
}

\author{
by \\ Slawomir Arkadiusz Krucinski M.Sc.(Eng.)
}

\author{
A Thesis \\ Submitted to the School of Graduate Studies \\ in Partial Fulfilment of the Requirements \\ for the Degree \\ Doctor of Philosophy
}

McMaster University

October 1989 
DESCRIPTION OF CLAY ANISOTROPY ... 
DOCTOR OF PHILOSPHY (1989)

(Civil Engineering

and Engineering Mechanics)
MCMASTER UNIVERSITY Hamilton, Ontario,

Canada

TTITLE: Description of Clay Anisotropy Employing the Concept of Directional Variation of Porosity.

AUTHOR: $\quad$ Slawomir Arkadiusz Krucinski

(M.Sc.(Eng.) - Technical University of Lodz, Poland)

SUPERVISORS: Dr. S. Pietruszczak and Dr. G.A. Oravas

NUMBER OF PAGES: xiii, 138 


\begin{abstract}
A mathematical framework has been developed for describing the effects of inherent and induced anisotropy in clays. The structure of governing equations permits, in general, the modelling of the sensitivity of soil response to the rotation of principal stress axes. The framework employs a continuum measure of material fabric, which is defined as an implicit function of the spatial distribution of porosity/void ratio. The set of classical functions describing the state of the material is thus enriched by new tensorial functions reflecting the orientation of the fabric. Such a formulation is advantageous over a conventional plasticity approach. It remains physically descriptive, in a sense that, the material response is a function of the specific manifestations of the microstructure.
\end{abstract}

The presented approach is general and its applicability extends to other geological materials, provided the proposed evolution law (Chapter 2) is appropriately modified and the material functions are adequately selected. In its present form, the formulation does not account for irreversibility of both the plastic flow and the evolution of microstructure auring histories experiencing stress reversals. However, the mathematical structure of the constitutive relations, as 
formulated in Chapter 3, is such that those effects can quite easily be incorporated into the proposed framework.

A key to full reliability of the proposed concept is a proper verification of the evolution law. The identification of the oriented fabric is not a simple matter and requires the use of complex experimental techniques. Some suggestions in that respect have been made in Chapter 2, where a general procedure for the estimation of the components of the porosity tensor from experimental observations has been proposed. 


\section{ACKNOWLEDGEMENTS}

The author is deeply grateful to his supervisors Dr. S. Pietruszczak and Dr. G.A. Oravas for their guidance, inspiration and encouragment during the course of this work.

Thanks are also due to the members of the Ph.D. Supervisory Committee: Dr. R. Sowerby and Dr. D. Stolle for their interest and valuable advice.

The financial support of the Natural Science and Engineering Research Council of Canada and McMaster University is gratefully acknowledged.

Finally, the author wishes to thank his wife for the patience and understanding throuhgout the whole period of this study. 


\section{TABLE OF CONTENTS}

PAGE

ABSTRACT

ACKNOWLEDGEMENTS $\quad$ v

LIST OF FIGURES $\quad x$

LIST OF TABLES $\quad$ xv

$\begin{array}{llll}\text { CHAPTER } & 1 & \text { Introduction. } & 1\end{array}$

$1.1 \quad$ General remarks. 1

1.2 Phenomenological considerations on

the internal structure of soils. 3

1.3 Review of experimental data

pertaining to anisotropy in clays.

1.4 Scope and objectives of the present research.

CHAPTER 2 Directional porosity as an implicit measure of inherent and induced soil anisotropy. 
$2.1 \quad$ Introduction.

2.2 A continuum concept of a measure

of internal structure; definition and mathematical description.

2.3 Sample estimation of porosity tensor based on available microscopic evidence.

CHAPTER $3 \quad$ Plasticity formulation for anisotropic particulate media.

3.1 Introduction; yield criteria for anisotropic media.

3.2 Plasticity framework for materials with inherently anisotropic fabric.

3.3 Description of anisotropy of plastic flow in materials with small grain size. 


$\begin{array}{lll}\text { CHAPTER } 4 & \text { Numerical simulations of anisotropy } \\ & \text { effects in normally and lightly } \\ & \text { overconsolidated clays. } & 71 \\ 4.1 & \text { Introduction. } \\ 4.2 & \text { Preliminary definitions; invariant } & 71 \\ & \text { measures of the effective stress } \\ & \text { state. } & 72 \\ 4.3 & \text { Specification of the yield function. } \\ 4.3 & \text { A numerical procedure for } \\ & \text { integration of constitutive relation. } \\ 4.4 & \text { Identification of material } \\ & \text { parameters. } & 81 \\ 4.4 .1 & \text { Weald clay. } & 86 \\ 4.4 .2 & \text { Spestone kaolin. } \\ 4.5 & \text { Numerical simulations; anisotropy } \\ & \text { induced by Ko-consolidation } \\ & \text { process. } & 89 \\ & \text { Conclusions. }\end{array}$


CHAPTER 5 On sensitivity of soil response to the rotation of principal stress directions. 103

$\begin{array}{lll}5.1 & \text { Introduction. } & 103\end{array}$

5.2 Theoretical considerations. 104

5.3 Numerical simulations of tests with the rotation of principal stress directions.

$\begin{array}{lll}5.6 & \text { Concluding remarks. } & 116\end{array}$

CHAPTER 6 Final re marks a n d recommendations.

APPENDIX B Isotropic yield functions for the critical state concept. 


\section{LIS T O F FIG URES}

FIGURE

PAGE

1.1 Rotation of principal stress directions relative to the axes of material symmetry; (a) general outline, (b) hollow cylinder cell, (c) directional shear apparatus.

1.2 Results of undrained triaxial shear test following $\mathrm{K}_{0}$-consolidation (after Mitchell, 1972)

1.3 Stress-strain response during triaxial shear following three different anisotropic consolidations (after Khera and Krizek, 1968).

1.4 Effective and total stress paths obtained in hollow cylinder test on Florida-1 clay (after Saada and Bianchini, 1975).

1.5 Results of drained directional shear test on loose Leighton Buzzard Sand (after Arthur et al., 1981)

1.6 Results of undrained directional shear test on overconsolidated Boston Blue clay (after Jamiolkowski et al., 1985).

1.7 Influence of strain induced anisotropy in loose Leighton Buzzard sand; test completed by Arthur et al. (1981) in directional shear cell:

2.1 Unit sphere enclosing a representative volume of the material.

2.2 Unit vector $\underline{\mathbf{v}}$ of a circle and anit normal vector $\underline{\mathbf{u}}$ to the plane of the circle. 


\section{LIST OF FIGURES}

FIGURE

PAGE

2.3 Graphical representation of porosity, at different stages of consolidation of Champlain clay (after Delage and Lefebvre, 1984); voids are black and particles are white.

2.4 A discus part of the cross-section, from Figure 2.3, subtracted for a purpose of lineal analysis.

2.5 Spatial distribution of void ratio for intact and consolidated sample of Champlain clay.

4.1 Typical yield surface for soils in the principal stress space.

4.2 Deviatoric cross section generated by equation (4.9) for different values of K (Jiang and Pietruszczak, 1988).

4.3 Meridional cross section of the yield function described by equation (4.10).

4.4. Flow chart of numerical code for integration of the proposed elastoplastic constitutive law.

4.5 Effective stress paths for $\mathrm{K}_{0}$-consolidation followed by undrained uniaxial compression.

4.6a Effective stress paths for $\mathrm{K}_{0}$-consolidation followed by undrained uniaxial compression.

4.6b Evolution of spatial distribution of void ratio; numerical simulation based on equation (2.24).

4.7: Hypothetical response during hydrostatic compression following $\mathrm{K}_{0^{-}}$ consolidation and undrained unloading. 


\section{LIST OF FIGURES}

FIGURE

PAGE

4.7b Evolution of spatial distribution of void ratio (numerical simulation based on equation (2.24).

4.8 Undrained compression following $\mathrm{K}_{0}$-consolidation and unloading; (a) effective stress paths, (b) material characteristics.

4.9 Stress probe experiment; direction of plastic strain increment.

102

5.1 Elastic and total strain generated by the pure rotation of the principal stress system following $\mathrm{K}_{0}$-consolidation; (a) total strain, (b) elastic strain.

5.2 Evolution of spatial distribution of void ratio under the pure rotation of the principal stress system.

5.3. Uniaxial undrained compression of $\mathrm{K}_{0}$-consolidated specimens with the different rate of the rotation of principal stress axes; (a) effective stress paths, (b) evolution of spatial distribution of void ratio.

5.4. Stress-strain response during undrained compression combined with a continuous rotation of principal stress axes. 


\section{IS T OF T A B LES}

TABLE

PAGE

1.1 Results of undrained shear test on Florida-1 Clay (after Saada and Bianchini, 1975).

2.1 Calculation of porosity distribution for the intact sample.

42

2.2 Calculation of porosity distribution for the consolidated sample $(421 \mathrm{kPa})$. 


\section{CHAPTER 1}

\section{INTRODUCTION}

\section{$1.1 \quad$ General Remarks.}

Most of soils are, in general, softer than other construction materials. Therefore, in the geotechnical design the load must be limited to the value constrained by the allowable deformation, rather than the bearing capacity of soil (Casagrande, 1932). In addition, the excess of the pore water pressure, and thus the effective stresses are the function of the previous deformation history. Consequently, even in the design based on the shearing resistance, the constitutive relation is required, unless it is assumed that shearing strength is independent of the deformation experienced by the material in the past. An attempt to predict the bearing capacity of piles or the stability analysis of slopes exemplify the most common situations, in which the application of overly simplified models that disregard the complex behaviour of soils, can seriously affect the foreseen results. Simultaneously, the advances in the testing techniques and instrumentation, as well as the rapid progress in computer technology and numerical methods, have created the opportunity for more rational geotechnical design. The assessment of the 
deformation of the soil mass can be obtained by the analysis of the proficiently defined boundary value problem of continuum mechanics. Such an analysis, regardless of numerical techniques employed, requires an appropriate constitutive relation. The mathematical sophistication of a constitutive model is the price to be paid for any endeavour to represent the reality more precisely. Thus, the applicability of the constitutive relation employed is always evaluated by an engineer and it calls for his personal professional judgment and experience.

The research presented here is concerned with the introduction of a new tensorial measure for the soil fabric. This measure is subsequently incorporated into the framework of plasticity, in order to arrive at a more realistic and rational description of geomaterials. While the applicability of the proposed concept extends to various geological media, the present research focuses mainly on mechanical behaviour of clays. Before proceeding to the details of such formulation it is necessary to concentrate, for a moment, on some aspects related to the description of internal structure of clays. Such a discussion may be instructive in the context of subsequent mathematical deliberations. For general considerations it is worthwhile to examine clays if they were a specific type of rock. Such a viewpoint is beneficial in the sense that the conclusions drawn can be applicable to the mathematical modelling of other geological materials. Consequently, the term 'rock' is used in its broadest sense, meaning any substance of the Earth crust interacting with an engineering structure. 


\subsection{Phenomenological Considerations on the Internal Structure of Soils.}

Rocks are conveniently classified into three main groups according to the method of formation (e.g. Attewell and Farmer, 1976; Blyth and De Freitas, 1984): igneous, sedimentary and metamorphic. Igneous rocks are formed by cooling of molten rock magma. Sedimentary rocks, like clays and sands, consist of mineral particles derived by weathering of a rock mass or/and particles formed by growth activities of animals and plants. The sediments are sorted during a process of transport, and after their settling become hardened owing to the action of the gravity forces and cementation processes. Finally, the metamorphic rocks are generated by alteration of an existing rock mass caused by variation in environmental conditions, mainly: temperature, pressure and chemical composition. Such a generally accepted classification creates an impression that the rock-forming process has been completed, whereas Bowen (1922), after his researç on reaction series, pointed out that,"... there is nothing tinal about rock forming. After, so called complete consolidation of rock many changes may occur between minerals, which are ordinarily termed as metamorphism and digenesis; new minerals may be formed and new structure may be imposed during these processes. ..." Owing to the disturbance in environmental conditions, often generated by engineering activities (e.g.: reduction or increase in a geostatic stress field, temperature changes, variations in a chemical composition of pore water and water content by itself), the processes similar to the metamorphism and digenesis may proceed in a vicinity of 
an engineering structure. If so, the rock structure may be significantly altered and this will undoubtedly influence its mechanical performance, as observed on a macroscale.

The atoms in both the minerals and rock forming elements, i.e., mineral grains and particles, are fitted together by the structural forces developed during the forming processes. It is generally accepted, that engineering properties of rocks principally depend on the character of these forces and the space composition of the rock forming elements; collectively called as internal structure, or briefly structure of a rock (cf. Grabowska-Olszewska and Siergiejew, 1977). In hard rocks bonding is produced predominantly by the overlaying wave functions of the valence electrons (known as chemical bonding). Depending on the configuration of the electrons in atoms, three types of chemical bonding can be identified: ionic, covalent, and metallic (cf. Grabowska-Olszewska and Siergiejew, 1977). The chemical bonding is typical for rocks with crystalline structure and is created when interactive atoms are close to each other, i.e., the distance between them is in the range of 0.5 to $3.5 \mathrm{~A}^{\circ}$. When the distance becomes larger the energy of bonding rapidly decreases. In loose sediments the distance between particles is usually not small enough to produce chemical bonding. Such structure is held together by the inter-particle forces (primarily of the hydrogen and van der Waal's type), a number of secondary forces arising from electric and magnetic fields surrounding clay particles and the forces generated by the presence of ions and water adjacent to the surface of clay particles. The energy of this type of bonding is much smaller than 
that of a chemical bond. On the other hand, however, the inter-particle forces can operate over far larger distances and are often strong enough to determine the final arrangement of the sediment particles. In clays, the predominant role in the creation of structure is played by platy clay minerals. Clay plate aggregates and clusters build connectors between the larger grains of silt and sand. The whole internal structure of a clay may be quite spacious, as in normally consolidated clays (with large void ratio) and, at the same time, a clay may posses some amount of tensional strength, known as a cohesion.

Before inspecting deformation characteristics on the macro level, it might be instructive to distinguish two types of solid structure, i.e., a 'compact' and an 'open' one. In compact structure, typical for metals, each atom has close neighbours and the arrangement of atoms is usually quite regular (at least in crystalline solids). In solids with open structure, like rocks, atoms within each particle are arranged in the same way as in 'compact' solids, but the particles themselves are connected together in an irregular manner, and often at rather far distances on an atomic scale (Chalmers, 1982). On macroscopic level, the open structure manifests itself primarily in the difference between the density of soil phase and the density of the bulk mass of forming elements. In addition, the material properties are sensitive to the confining pressure.

Atoms in crystals have a specific separation at which the interatomic forces are in equilibrium. An action of an external load perturbs this equilibrium and 
atoms move apart in order to reach the new balance of interatomic forces. When the load is released atoms move back to their original state, hence the observed macroscopic behaviour is elastic. Unfortunately, this mechanism of deformation is purely speculative, since materials like perfect crystal are very rare, if they really exist at all. Most of the engineering materials contain a certain amount of imperfections, such as: dislocations, pores, fissures, etc., which are considered to be the source of irreversible deformations. In the compact policrystaline materials, such as metals, it is believed that dislocation movement and its growth is a major mechanism of irreversible deformation, unless the extent of dislocations reaches the saturation level and the material becomes brittle (Chalmers, 1982). In materials with open structure, such as rocks, although the deformation process of rock forming minerals might be similar to that in metals, the overall mechanism is much different owing to the presence of weaker bonding and larger porosity. From an early stage, the deformation process is influenced by the nucleation of fissures and the evolution of voids, which results in splitting of grains and clusters. The irreversible deformations are generated mainly by the relative sliding, rolling and lifting of particles (Mitchell, 1976; Rowe, 1962), since the structural forces between them may be broken much easier than the interatomic forces of mineral crystals. Hence, the hardening process of these materials is a result of a modification in granulation and an increase in grain compaction (an average contact force between grains decreases with the number of grains and the load required to continue the progressive deformation increases with wedging of particles). One can expect that, the evolution of internal structure of the solid plays a significant role in the 
deformation process. On the macroscopic level the rock structure manifests itself by the directional dependence of both the strength and the deformation characteristics. The experimental findings on the internal structure of soils and its relation to the anisotropy in the mechanical properties are discussed in the following section.

\subsection{Review of experimental data pertaining to anisotropy in clays}

Deviations from isotropy (loosely defined as directional dependence of deformation characteristics) in natural soil deposits and rocks are already well documented in the literature (e.g. Mitchell, 1972; Ochiai and Lade, 1983; Ingles and Lee, 1971; Douglass and Voight, 1969). The following review of experimental observations is mainly devoted to clays, because their behaviour is of a primary objective. However, some auxiliary comments on anisotropic effects in sands are also given, in the light of certain analogies in the micro and the macro mechanical performance of both types of soils.

In order to investigate the anisotropic strength-deformation characteristic of a material one must allow for the rotation of principal stress directions, relative to the axes of material symmetry (see Figure 1.1). In the early experiments pertaining to the anisotropy of clays behaviour (Khera and Krizek, 1968; Mitchell, 
1972), the rotation of principal axes of stress was achieved by cutting the specimens of apparently homogeneous soil at different inclinations to the major consolidation stress, or the direction of the deposition, and subsequently compressing it in a conventional triaxial cell. Mitchell (1972) completed a number of drained and undrained triaxial compression tests on vertical and horizontal specimens trimmed from a one-dimensionally consolidated block of kaolin. Prior to the uniaxial compression, the specimens were isotropicly reconsolidated by the confining pressures both less and grater than the vertical pressure used in preparing the block sample. The fully drained tests exhibited no significant difference in shear strength between vertical and horizontal specimens, whereas in some of the undrained tests on overconsolidated specimens deviations from isotropy were detected. The vertical and the horizontal specimens rebounded above the original consolidation pressure exhibited behaviour very similar to specimens tested after the initial isotropic consolidation. Typical deformation characteristics and stress paths obtained by Mitchell (1972), are reproduced in Figure 1.2. Another, quite extensive study concerning the effects of various stress histories on the undrained response of the inclined specimens of an illite clay was reported by Khera and Krizek (1968). Specimens trimmed parallel (V series) and normal (H series) to the direction of the one-dimensional consolidation (up to $80 \mathrm{kPa}$ ) were subjected to: (1) isotropic consolidation, and $(2,3)$ anisotropic consolidation under nominal principal stress ratios of 1.5 and 2.0 , respectively. The major principal reconsolidation stress was always applied parallel to the axis of triaxial specimen, whereas the mean effective stress achieved was of the order of $100 \mathrm{kPa}$. All specimens were subsequently failed 
by increasing the axial load, while maintaining the cell pressure constant and prohibiting drainage. The results reproduced in Figure 1.3, clearly indicate that the undrained strength is highest in vertical specimens. At the same time, the stiffness moduli prior to failure show a strong directional dependence. The reconsolidation process influences the strength as well as the deformation characteristics of the both series. However, its effect is more pronounced when the direction of minor principal stress coincides with the direction of the previous Ko-consolidation.

Although, the anisotropy in the behaviour of both normally consolidated and overconsolidated clays is quite evident, the quantitative conclusions drawn from these results (i.e. Figure 1.2 and 1.3) are speculative (Saada and Bianchini, 1975). The major concern is the necessity of the removal of the initial stress system under which the clay was originally formed, in order to be able to trim the inclined specimens (structure of clay can evolve during unloading and a specimen can not be reconsolidated to exactly the same depositional conditions). In addition to that, the bending moments and shearing forces are generated at the top and the bottom of a recompressed specimen owing to the presence of anisotropy, which may obliterate the true anisotropic response (Saada and Bianchini, 1977). In order to overcome these deficiencies new testing equipment has been gradually developed, such as the hollow cylinder cell, and the directional shear cell. Some limited studies have also been successfully completed using the true triaxial apparatus. Although, in the latter case only the normal and parallel orientation of the major loading stress relative to the axes of primary consolidation can be achieved. 
In the hollow cylinder cell a specimen is subjected to a combination of an axial and a torsional stress in a constant ratio. As a result, the inclination of the major principal stress is also kept constant during a test. The influence of the end effects, associated with the use of inclined specimens, is partially eliminated by selecting the specimen of appropriate height (Saada and Townsend, 1981). Similarly like in a triaxial cell, the intermediate principal stress is always radial and equal to the chamber pressure. A comprehensive study on the behaviour of undisturbed and Ko-consolidated clay specimens, employing the described device, have been reported by Saada and Bianchini (1971). The specimens were tested under the undrained condition and the prime objective was to investigate the influence of the orientation of the principal stress axes on the form of the failure criterion and on the pattern of effective stress paths. The analysis of 130 tests indicates that every orientation of the principal stress system results in a different effective stress path (Figure 1.4), i.e., clays with the same water content but different degree of anisotropy respond differently to the same stress histories. Moreover, a substantial scatter in the angle of internal friction, in terms of effective stresses, was obtained as indicated in Table 1.1 (cited after Saada and Bianchini, 1975), which suggests an influence of inherent and induced anisotropy on the failure criterion. The adoption of the hollow cylinder cell, and its modified version which allows for the application of the unequal inner and outer pressures on the hollow specimen sides (Symes, Gens and Hight, 1984), offers some improvements in the testing technique. However, the repeatability of experiments and the influence of non-uniformities in the strain and the stress field on the quality of the results are still of concern to investigators (Jamiolkowski, 
Ladd, Germaine and Lancellotta, 1985).

Future advances in experimental technique pertaining to soil anisotropy are foreseen in recently developed directional shear apparatus. In this apparatus the direction of the major principal stress is controlled by varying the normal and shear stress acting on four faces of a cubical sample placed between the rigid end platens. During a test, the detailed information about strain distribution is provided by recording either the surface displacement field or the radiographic-photographic tracing of the shot displacements embedded in a specimen. Figure 1.5 and 1.6 show the results of a series of directional shear test performed on loose sand and resedimented clay (Jamiolkowski et. al, 1985). The results clearly indicate the sensitivity of elasto-plastic behaviour and the shear resistance to the inclination of the directions of the principal stress system relative to the symmetry axis of specimen tested. Another interesting experiment illustrating the influence of the strain induced anisotropy was completed by Wong and Arthur (1985). The drained test was run on the specimen of a loose sand, initially prestrained in shearing with a fixed major principal stress direction. Subsequently, the specimen was unloaded and repeatedly sheared with major principal stress at a different direotion. Figure 1.7 shows the results of the described loading programme. The strain induced anisotropy results in much stiffer response at the small rotation angles and much softer one at the more advanced rotation. Furthermore, it should be noted that inherent anisotropy generates a continuous degradation in stiffness as the direction of major principal stress rotates from $0^{\circ}$ to $90^{\circ}$ (Figures 1.5 and 1.6). On the 
other hand, the strain induced anisotropy reduces stiffness to a minimum at the rotation of about $70^{\circ}$, and then it results in progressive increase (Figures 1.7). The similar effects were also observed in experiments on dense sands (Arthur, Chua and Dustan, 1977; Arthur, Bekenstein, Germaine and Ladd, 1982)

Anisotropy in the mechanical response of clays, as observed on a macroscale, is a manifestation of internal soil structure. An alignment of soil particles has been detected in various natural clay and sand deposits (e.g.Delage and Lefebvre, 1984; Duncan and Seed, 1966; McConnachi, 1974; Mitchell, 1972), and it appears to be induced by both deposition: and environmental conditions. Microscopic evidence suggests that the preferred orientation in soil particles can also be achieved during the one-dimensional Ko-consolidation process (Delage and Lefebvre, 1984; McConnachi, 1974). Therefore, the problem of anisotropy entails identification of the initial soil structure, as linked to the inherent anisotropy, and its evolution owing to imposed deformation and environmental disturbances, as associated with the induced anisotropy.

A large number of investigations on correlation between microscopic observations and the mechanical performance of soils have been performed since the electron-microscopy was developed (e.g. Barden, 1972; Delage and Lefebrve, 1984; McConnachi, 1974; Mitchell, 1972). It has been speculated that micromechanical processes in clays and sands might be considered as to be similar (Barden, 1972). Commonly adopted single plate clay structure has been questioned. 
It become evident that such a structure is only relevant to dilute colloidal suspensions, whereas in natural clay deposits multiplied unit (cluster) structure is usually observed (Barden, 1972; Pusch, 1970). Therefore, the macroscopically detected anisotropy in shear strength of clays can be attributed to the size and bias in the orientation and the shape of clay clusters. This observation can partially explain the dual behaviour of some clays, i.e., an anisotropic deformation mode and the isotropy in the failure criterion. One can speculate that, the anisotropy in shear strength of clay, as described by the failure criterion in terms of effective stresses, can be associated with the cluster size. On the other hand, the deformation characteristics can be solely affected by the arrangement of clay plates.

The main problem in correlating the soil structure with its macroscopically observed performance is the lack of the commonly accepted measure of the structure. Based on the existing experimental evidence, it seems logical to relate the directional dependence of the soil and rock behaviour to the distribution of pores and fissures (e.g. Delage and Lefebvre, 1984; Ingles and Lee, 1971; Douglass and Voight, 1969). This suggests that the spatial distribution of voids can be adopted as an implicit measure of soil fabric. In the scalar form the phase distribution in geomaterials is defined through porosity or void ratio. Consequently, in the subsequent development the concept of 'directional' porosity or the 'directional' void ratio is introduced. This quantity is ciefined in proper mathematical terms and it is proposed as an implicit measure of soil structure. 


\subsection{Scope and Objectives of the Present Research.}

The presence of a non-random fabric, perceived as a nonhomogeneity of the material, creates the anisotropy in the mechanical response of soils. Depending on the scale of the problem, there are essentially two different approaches for the modelling of nonhomogeneous soils. In the case of a wide spacing, as compared to a problem studied, the soil mass can be modeled by an assemblage of building blocks (mega-elements), formed by intersections of discontinuities in the soil mass. The presences of fissures and joints, packed by a gouge material, is accounted for by introducing the isolated discrete joint elements or the thin layer elements (Goodman, Taylor and Brekke, 1968; Pande and Sharma, 1977; Desai, 1985). Usually, only a few large scale discontinuities intersect the soil mass and the number of the elements can be kept within the reasonable limit. The key question of the analysis is to calculate the modes of the block movement and to find the most hazardous zones in the soil mass, when subjected to the external loads. When discontinuities are present in a very large number, and their spacing is close, the individual modelling of their influence on the entire soil mass is impractical. Therefore, an alternative approach of an 'equivalent continuum' may be adopted either via empirical relations (Amadei and Goodman, 1981), or by assuming that plastic flow is localized along the planes of discontinuities (Pietruszczak and Stolle, 1986). In the latter case, the mechanical response of soil element is affected by the mechanical properties of both the intact material and the faults, as well as by the geometrical aspects of the discontinuities, i.e. the spacing and the scattering in 
discontinuity orientations about the mean direction.

The objective of this thesis is to develop a rational plasticity formulation for anisotropic particulate media, which is capable of describing the effects of inherent and induced anisotropy in clays. The formulation employs the concept of an 'equivalent continuum' and introduces a new measure of material fabric. Thus, the set of classical functions representing the state of the material is enriched by a new tensorial function. Such a formulation is advantageous over a conventional plasticity approach since it remains physically descriptive, i.e., the material response is an implicit function of the microstructure of the material.

In Chapter 2 the concept of fabric tensor is first introduced. Next, the measure of spatial distribution of the porosity is defined in the form of the surface integral of a vector field over a representative volume. The mathematical details concerning the 'porosity tensor' are followed by the discussion of the evolution law for its components. Finally, a procedure for the estimation of the components of the porosity tensor from experimental observation is proposed, and illustrated by an example based on the available measurements.

Chapter 3 pertains to plasticity formulation for anisotropic soil. The chapter begins with the review of the previous attempts of describing anisotropy of plastic flow. Subsequently, the concept of fabric tensor, assumed as an implicit function of 'directional porosity', is incorporated into the plasticity framework. 
Various possible forms of the anisotropic elasto-plastic constitutive relation are derived and examined. The correspondence of the developed relations with the non-associated plasticity and the kinematic hardening model is discussed.

Chapter 4 is concerned with numerical verification of the proposed framework. First, the form of yield surface is specified in the invariant stress reference frame. Subsequently, the numerical simulations pertaining to normally consolidated and lightly overconsolidated clays are presented and examined against the experimental data. This chapter includes also the description of the procedure developed for numerical integration of the elasto-plastic constitutive relation.

In Chapter 5 the effect of rotation of the principal stress system on the resulting displacement filed is discussed. It is demonstrated that most well established plasticity models do not account for this influence. Ultimately, the capability of the proposed constitutive relations to model the sensitivity of soil response to the rotation of principal stress axes is examined and illustrated by some numerical examples.

Finally, in Chapter 6 the basic conclusions from the presented research are recapitulated and some recommendations for future investigation are made. 
a.

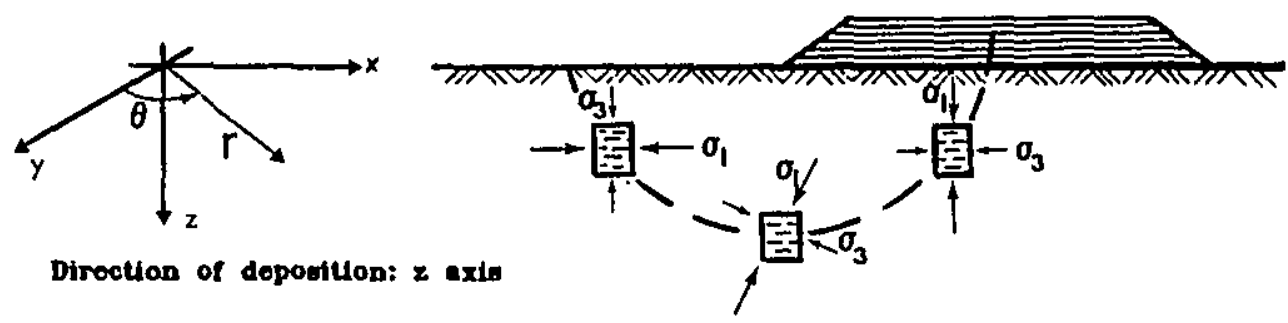

b.

Load imposed on a sample Stresses on an element in the wall
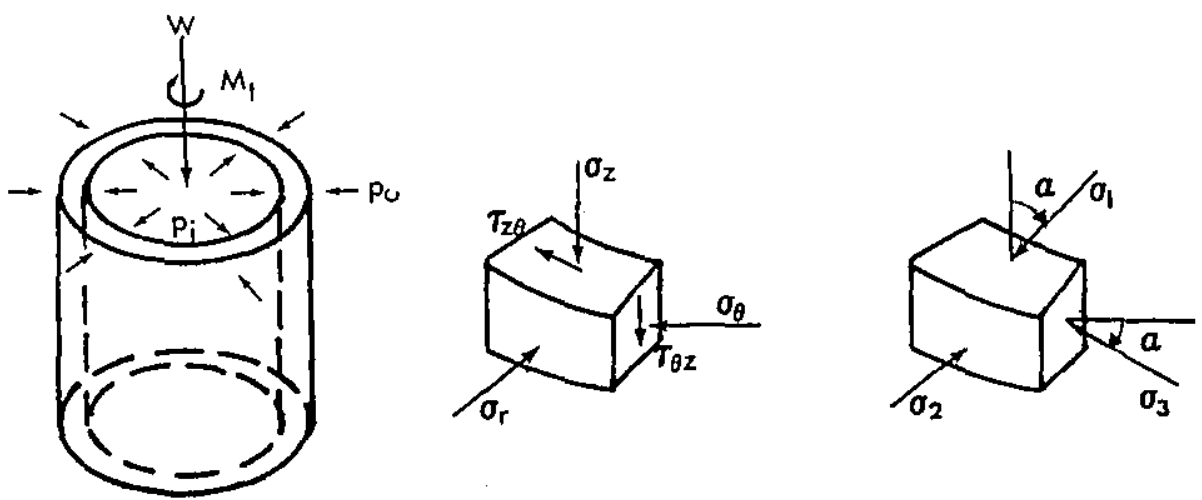

C.

Stresses imposed on a sample

Test in $x-z$ plane

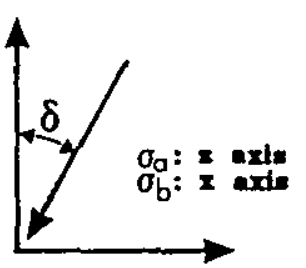

Test in $x-y$ plane

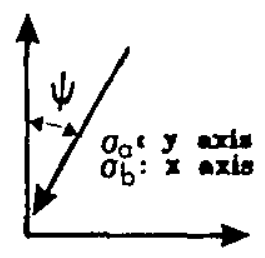

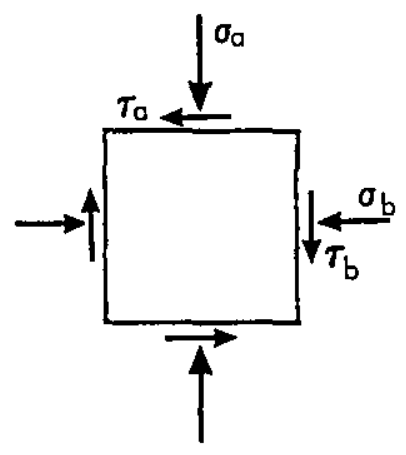

Figure 1.1 Rotation of principal stress directions relative to the axes of material symmetry; (a) general outline, (b) hollow cylinder test, (c) directional shear test. 


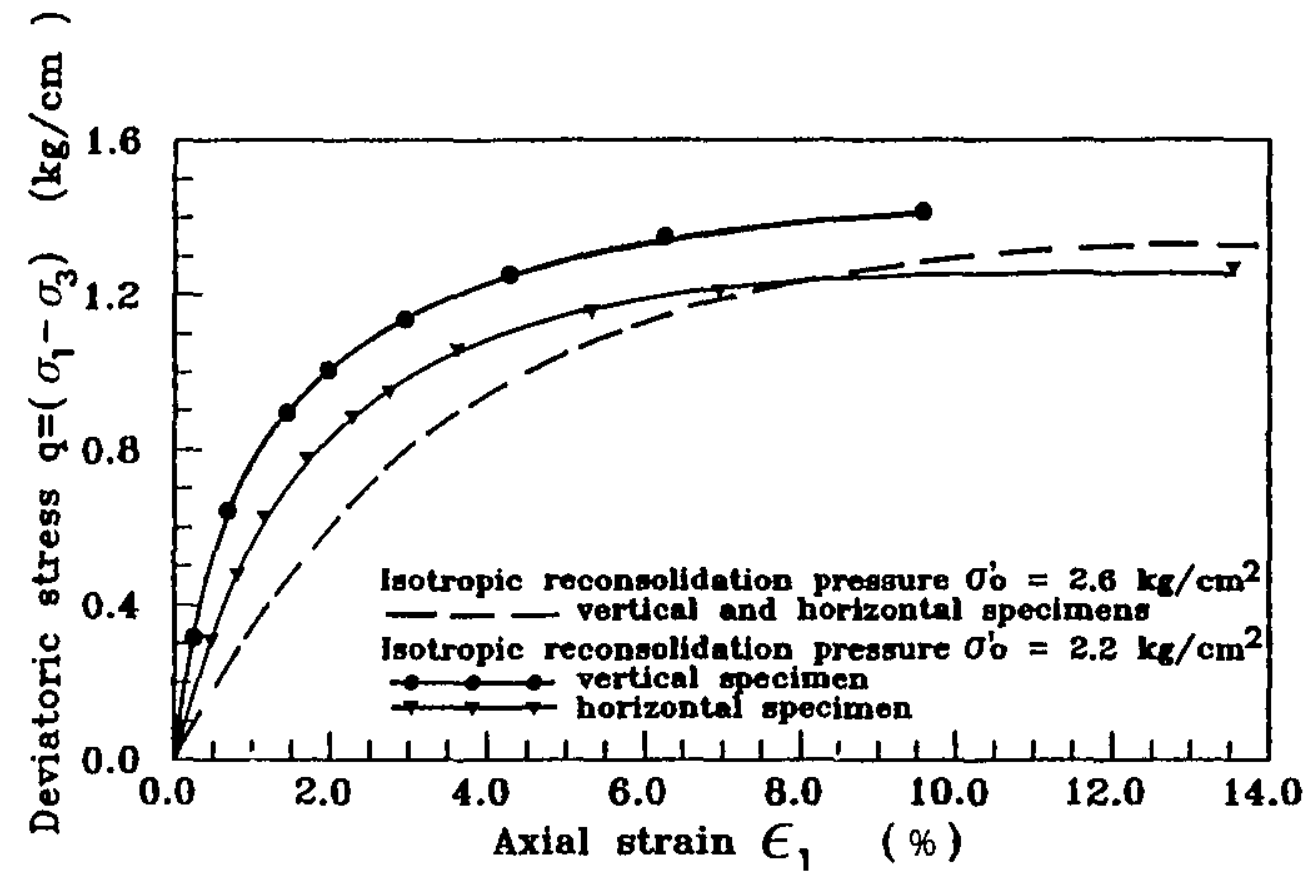

Figure 1.2 Results of undrained, triaxial shear test following Ko-consolidation at $\sigma_{\mathrm{v}}^{\prime}=2.5 \mathrm{~kg} / \mathrm{cm}$ (after Mitchell, 1972). 

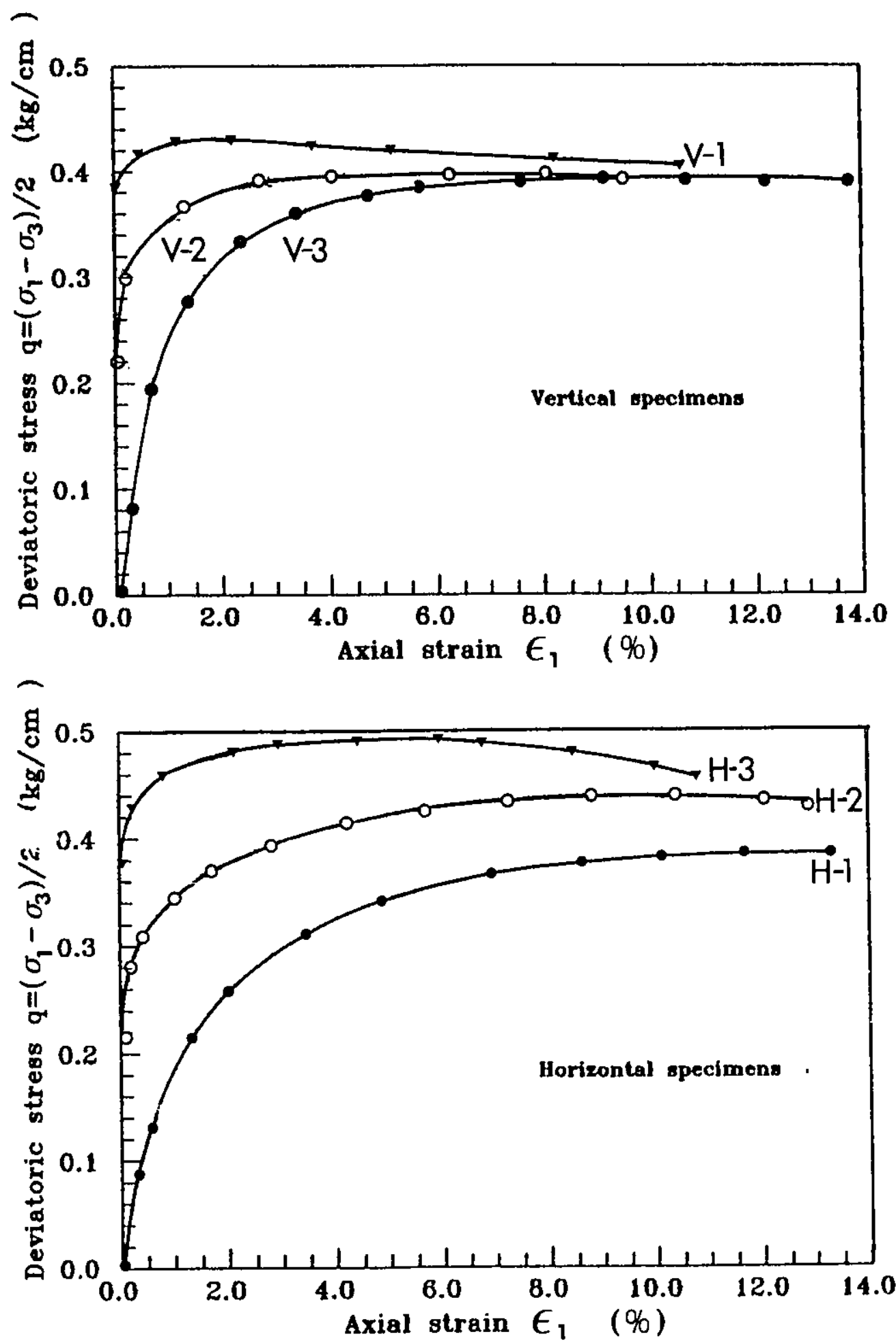

Figure 1.3 Stress-gtrain response during triaxial shear following three different anisotropic consolidations (after Khera and Krizek. 1968) 

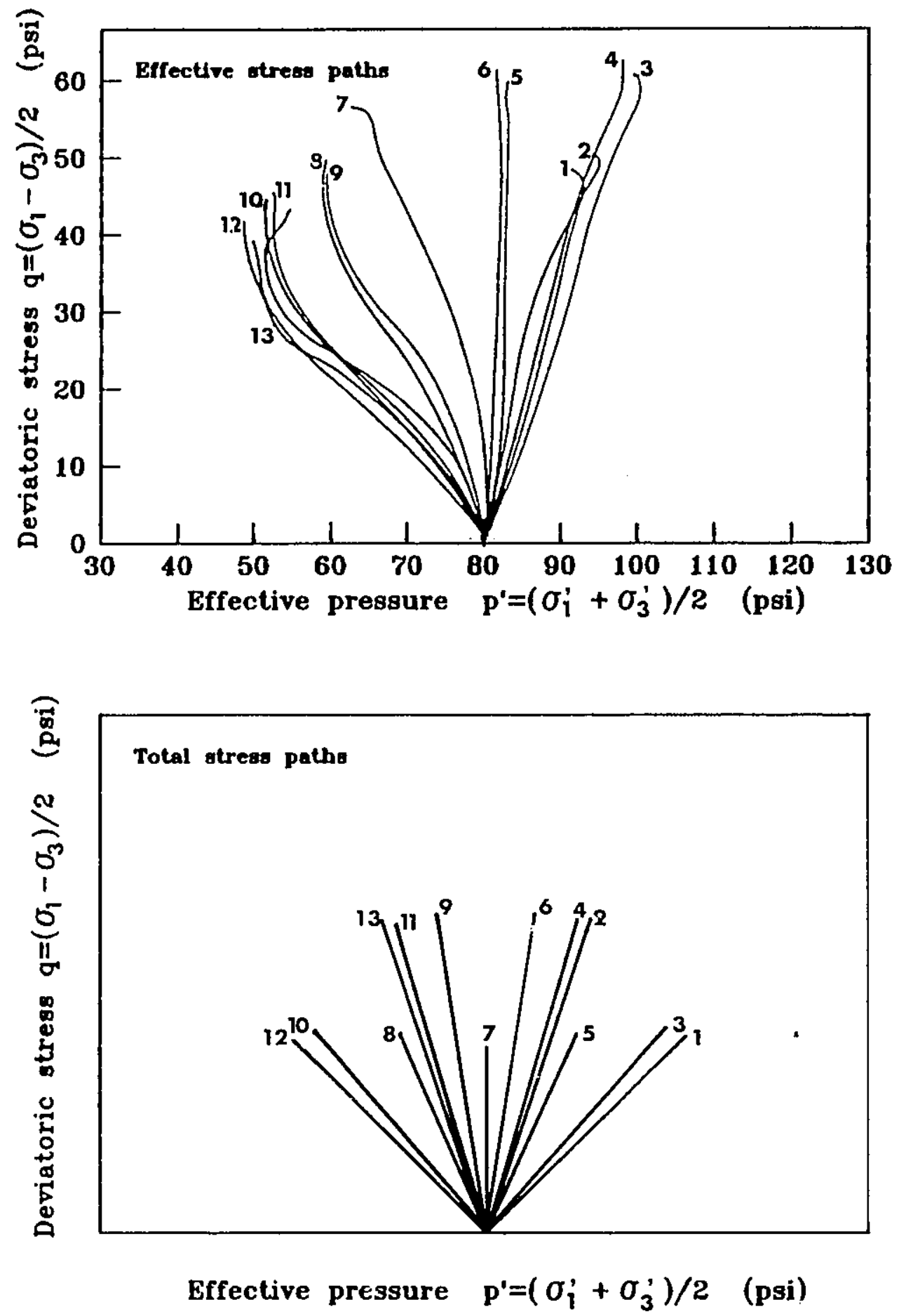

Figure 1.4 Effective and total stress paths obtained in hollow cylinder test on Florida-1 Clay (after Saada and Bianchini. 1975) 
Table 1 Results of undrained shear test on Florida-1 Clay

\begin{tabular}{|c|l|l|c|}
\hline $\begin{array}{c}\text { Test } \\
\text { number }\end{array}$ & Type of test & \multicolumn{1}{|c|}{$\alpha$} & $\mathrm{q}_{\mathrm{f}}=\left(\sigma_{1}-\sigma_{3}\right) / 2$ \\
& & degree & $\mathrm{psi}$ \\
\hline 1 & $\mathrm{DC}$ & 0. & 39.8 \\
2 & $\mathrm{GC}$ & 0. & 41.0 \\
3 & $\mathrm{DCR}$ & 15. & 49.9 \\
4 & GCR & 15. & 51.6 \\
5 & DCR & 31.75 & 49.1 \\
6 & $\mathrm{GCR}$ & 31.75 & 50.5 \\
7 & $\mathrm{R}$ & 45. & 46.2 \\
8 & DTR & 58.25 & 40.5 \\
9 & GTR & 58.25 & 39.4 \\
10 & DTR & 75. & 36.3 \\
11 & GTR & 75. & 37.3 \\
12 & DT & 90. & 34.2 \\
13 & GT & 90. & 32.4 \\
\hline Consolidation cell pressure $=80 \mathrm{psi}$ & \\
Ko = $0.48 ; \mathrm{w}=35.23 \%, \mathrm{w}_{\mathrm{L}}=62.5 \%, \mathrm{w}_{\mathrm{p}}=39.0 \%$ \\
\hline
\end{tabular}




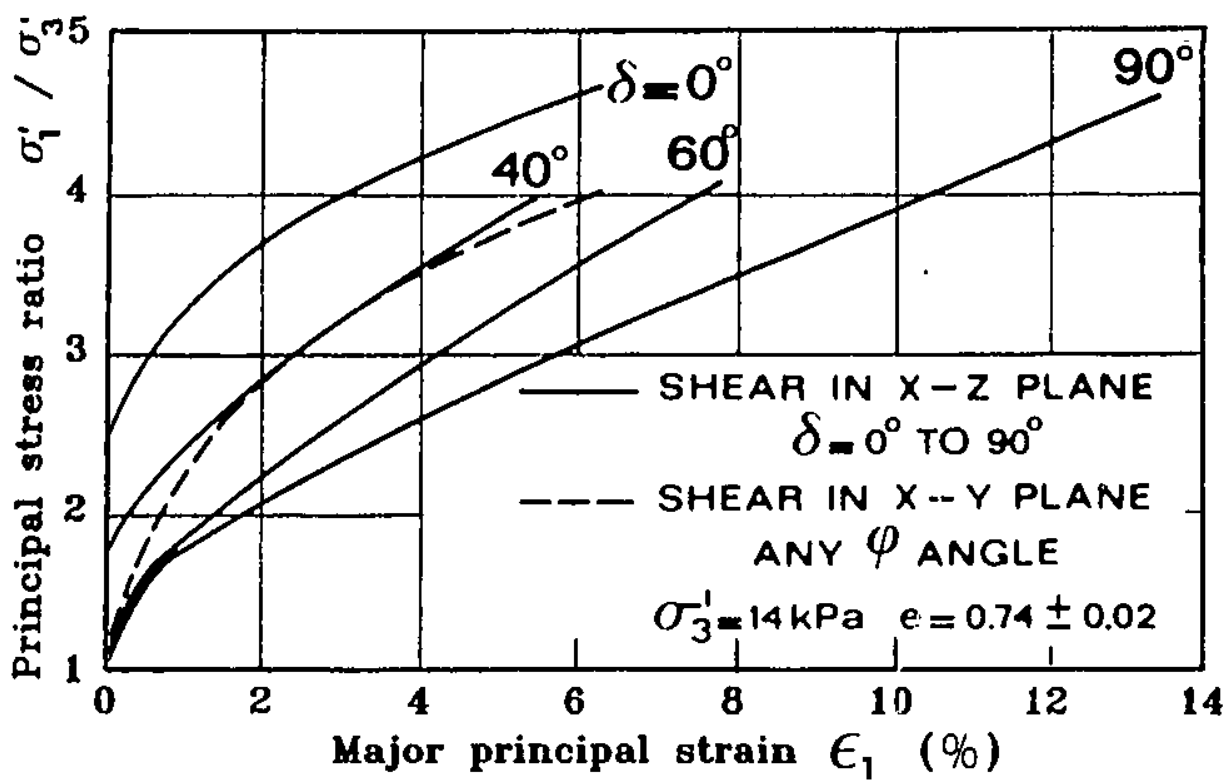

Figure 1.5 Results of undrained directional shear test on loose Leighton Buzzard Sand (afier Arthur et al., 1981)

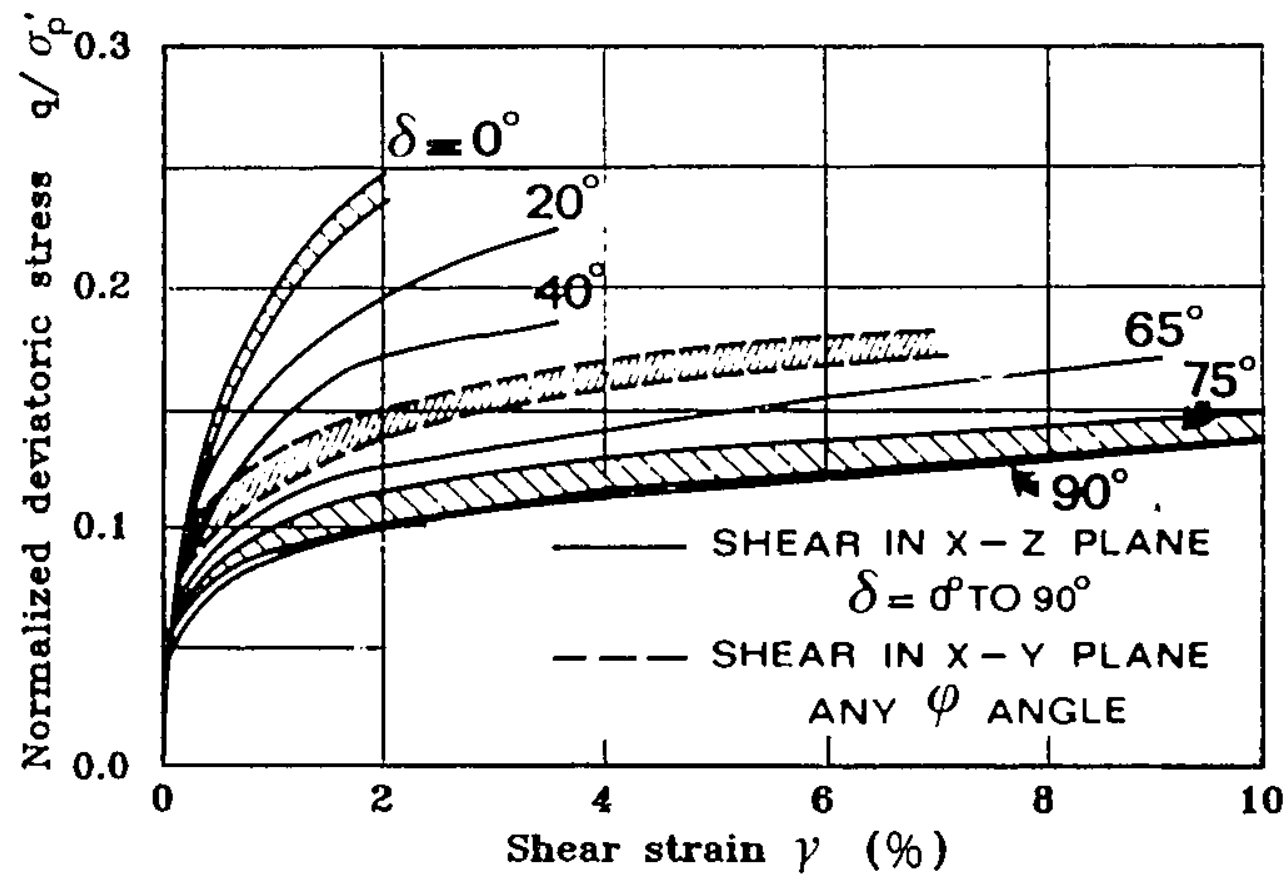

Figure 1.6 Results of undrained directional shear test on overconsolidated Boston Blue Clay (after Jamiolkowski et al., 1985) 


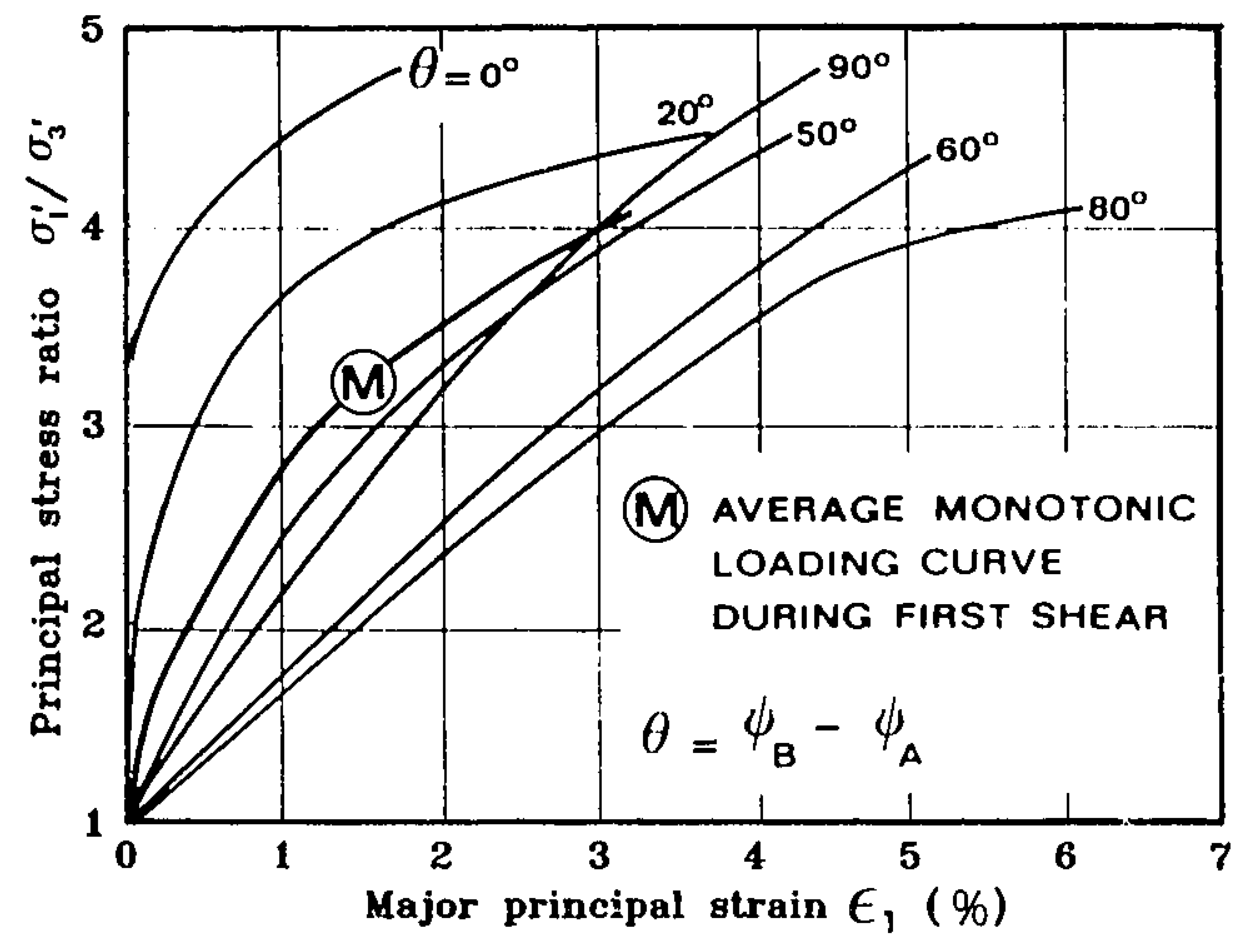

Figure 1.7. Influence of strain induced enisotropy in loose Leighton Buzzard Sand; test completed in directional shear cell by Arthur et pi. (1981) 


\section{CHAPTER 2}

\section{DIRECTIONAL POROSITY AS AN IMPLICTT MEASURE OF INHERENT AND INDUCED SOIL ANISOTROPY}

\section{$2.1 \quad$ Introduction.}

In principle, the term "soil structure" refers collectively to dimension, shape, quality of outer surface and relative composition of soil forming elements, i.e. mineral grains, aggregates, cementation substances, as well as to the nature of the bonding between these elements (cf. Grabowska-Olszewska and Siergiejew, 1977; Mitchell, 1976). As noted by Mitchell (1976) "... the term 'structure' is often used interchangeably with the term 'fabric' ..., however the former ... has the broader meaning since the fabric usually refers to the arrangement of the forming elements and pore spaces in a soil ...". It should also be noted that, the geometrical (or spatial) composition of the soil structure is defined by some authors as the soil "texture" (Grabowska-Olszewska, 1977).

In the context of the above definitions soil can be regarded as an abstract quantity, 'body', discerned as a set of noncountable material elements, i.e. particles 
in the physical space. The particles interact with one another and are placed in a field of external influence. In order to obtain a constitutive equation the inevitable assumptions have to be made, viz.,:

- the physical space is identified with three dimensional Euclidian space $\mathrm{E}_{3}$;

- the particles are assumed to be distributed continuously in the physical space, this means that the material always exists, even in an infinitesimally small region of space;

- time is the absolute monotonically increasing scalar measure between events;

- the interaction between particles and current thermodynamical state of material can be represented in a form of tensorial functions and functionals specified on the particles; thus, the relation between the mechanical agencies and a mechanical response of a material must remain indifferent to the material orientation in the space.

Although, strict homogeneity and continuity are never achieved in soils, it seems sensible for engineering purpose to consider soil as a homogeneous continuum, at least in a statistical sense, and to describe the effects of structural arrangement by means of some appropriate continuous measures. Thus, the set of classical functions reflecting the state of the material has to be enriched by a new tensorial function or functions which is or are capable of representing the soil fabric and its evolution.

The multiplicity of the measures of the internal structure were employed in the past; the review of some of them is provided by Krajcinovic and 
Lemaitre (1987). Most of these concepts were introduced intuitively, like Kachanov's 'damage tensor' (Vakulenko and Kachanow, 1971), which describes the evolution and the distribution of microcracks, or Oda's (1972) measure of the number of contacts between sand grains. The drawback of most concepts is the lack of the direct connection with the experimentally available stereological measurements used in geology and geophysics. In further considerations, an indirect measure of soil structure is introduced which can be estimated from the microscopic observation of soil (Pietruszczak and Krucinski, 1989). The proposed measure is continuous and non-singular, therefore, it can be easily incorporated into the mathematical framework of the theory of plasticity. Since, volume-phase composition is a well established and widely used characteristic of soil, an attempt is made to describe anisotropy of soil structure in terms of anisotropy of its phase distribution. As an implicit measure of the internal structure of soil, a 'directional porosity' is employed, which could be recognized as the generalized, directiondependent counterpart of 'porosity',i.e., the scalar valued quantity defining the fraction of the void space. The 'directional porosity' is constructed as the average, continuous and non-singular measure in the form of an integral over a representative volume, and it is mathematically expressed in terms of the generalized Fourier series. Its values can be estimated directly from experiment by means of quantitative microscopy, in particular by the lineal analysis, as illustrated in paragraph 2.3 . 


\subsection{A Continuum Concept of a Measure of Internal Structure: Definition and Mathematical Description}

Let us isolate in the vicinity of a material point $P$ the unit sphere $S(P)$, i.e., $R=1$ being the radius of the sphere, which limits a representative volume of the material $V^{*}$. Consider now a test line of length $L_{T}=2 R$ which is within the sphere, and which orientation in three dimensions is defined by spherical coordinates $\Phi$ and Ө, with respect to the Cartesian coordinate system fixed in the material, Fig. 2.1 . From the point of view of statistics, the sample space for this experiment consists of continuum of points on the surface of the sphere. Consider some small range of orientations specified by varying $\Phi$ and $\theta$ by amounts $d \Phi$ and $d \theta$, see Fig. 2.1 . If the test orientations are distributed uniformly over the sphere $S(P)$, the fraction which lies within the range $\Phi$ to $d \Phi$ and $\theta$ to $d \theta$ is equal, in the limit, to the fraction of the sphere which is occupied by the spherical image of the orientation range, dS $=R^{2} \sin \Phi \mathrm{d} \theta \mathrm{d} \Phi$. The spherical image of the whole range of orientations is $4 \pi \mathrm{R}^{2}$, hence the probability that an orientation selected at random lies within the range $\Phi$ to $d \Phi$ and $\Theta$ to $d \theta$, i.e., the combined frequency function for $\Phi$ and. $\theta$, is:

$$
\mathrm{f}(\Phi, \theta) \mathrm{d} \theta \mathrm{d} \Phi=\frac{\sin \Phi \mathrm{d} \Theta \mathrm{d} \Phi}{4 \pi}
$$


I.et

$$
l(\Phi, \theta)=\sum_{i} l_{i}(\Phi, \theta)
$$

be the total length of interceptions of the test line $L_{T}=2 R$ at the position $(\Phi, \theta)$ with soil porcs. The fraction of this test line occupied by pores is then defined as,

$$
\mathrm{L}(\Phi, \Theta)=1(\Phi, \Theta) / \mathrm{L}_{\top}
$$

The mean value of quantity $L(\Phi, \theta)$ averaged over the domain $S(P)$, i.e. in all directions of test lines, is the expected value of the random variable $L(\Phi, \theta)$ and, for continucus function of the variables $\Phi$ and $\theta$, is expressed by formula, cf. (Hilard, $1968)$

$$
L_{\mathrm{av}}=\int_{0}^{\pi} \int_{0}^{2 x} L(\Phi, \Theta) f(\Phi, \theta) d \theta d \Phi
$$

where $f(\Phi, \theta)$ is the joint probability of finding a test line within the range $\Phi$ to $\Phi+d \Phi$ and $\theta$ to $\theta+d \theta$. For uniformly distributed test lines, the substitution of equation (2.1) into equation (2.3) gives,

$$
L_{\mathrm{av}}=\frac{1}{4 \pi} \int_{0}^{\pi} \int_{0}^{2 \pi} \mathrm{L}(\Phi, \theta) \sin \theta \operatorname{d\theta } \dot{ } \dot{ } \Phi
$$

The last integral can be recognized as the surface integral of vector field $L(\underline{v}):=I,(\Phi, \theta)$ over the unit sphere,i.e, 


$$
\mathrm{L}_{\mathrm{av}}=\frac{1}{4 \pi} \int_{\mathrm{S}(\mathrm{p})} \mathrm{L}(\underline{\mathrm{v}}) \mathrm{dS}(\underline{\mathbf{v}})
$$

Here $\mathrm{y}$ is the unit vector normal to the surface of the sphere $\mathrm{S}(\mathrm{P})$. It is well known from vector analysis that the above integral can be calculated as the sum of integrals over the projection of $S(P)$ on three perpendicular planes defined by the coordinate system. Thus, in view of equation (2.2) the integral (2.4) can be expressed as,

$$
\mathrm{L}_{\mathrm{av}}=\frac{1}{8 \pi}\left(\iint_{\mathrm{S} y z} 1_{x} \mathrm{dydz}+\iint_{S z x} 1_{y} \mathrm{dzdx}+\iint_{S x y} 1_{z} \mathrm{dxdy}\right)
$$

The quantities $l_{x} d y d z, l_{y} d z d x, l_{z} d x d y$ are the volumes of pores contained within the bars of soil whose bases have area $\mathrm{dydz}, \mathrm{dzdx}$, dxdy and whose average altitudes are $l_{x}, 1_{y}, l_{z}$ accordingly. Therefore, the integral (2.6) is equivalent to six times the total volume of pores space $V_{v 0}$ enclosed by unit sphere $S(P)$. Moreover, since the term $8 \pi$ represents the volume of the unit sphere multiplied by six, the integral $(2.5)$ is the measure of average porosity $n_{0}$ in the neighborhood of the point $P$. Accordingly, the lineal fraction occupied by pores is an unbiased estimator of the volume fraction of voids, i.e.,

$$
\mathrm{n}(\underline{\mathbf{v}}) \equiv \mathbf{L}(\underline{\mathbf{v}}) \quad ; \quad \mathrm{n}_{0}=\mathrm{L}_{\mathrm{av}}
$$


The scalar valued function $n(\Phi, \theta)$ can be approximated by the generalized double Fourier series, (cf. Gelfand et al., 1963),

$$
\mathrm{n}(\Phi, \theta) \approx \Sigma \Sigma \mathrm{C}_{\mathrm{nm}} \Phi_{\mathrm{nm}}(\Phi, \theta)
$$

If a set $\Phi_{n m}(\Phi, \Theta)$ was selected as the system of orthogonal spherical harmonics, then the expansion (2.8) is prone to be the expansion of the type,

$$
\begin{aligned}
& n(\Phi, \theta) \approx n_{0}\left[1+\sum_{n=2}^{\infty}\left\{\frac{1}{2} a_{n 0} P_{n}(\cos \theta)+\right.\right. \\
& \left.\left.\sum_{m=1}^{n} P_{n}^{m}(\cos \theta)\left(a_{m m} \cos (m \Phi)+b_{m} \sin (m \Phi)\right)\right\}\right]
\end{aligned}
$$

where the expansion coefficients are as follows,

$$
\left[\begin{array}{l}
a_{m m} \\
b_{n m}
\end{array}\right]=\frac{2(n+1)}{4 \pi n_{0}} \frac{(n+m) !}{(n-m) !} \int_{0}^{2 \pi} \int_{0}^{\pi} L(\Phi, \theta) P_{n}^{m}(\cos \theta)\left[\begin{array}{c}
\cos m \Phi \\
\sin m \Phi
\end{array}\right] \sin \theta d \theta d \Phi
$$

Here, $P_{n}(\cos \theta)$ is the $n$-th Legendre polynomial, $P_{n}{ }^{m}(\cos \theta)$ is the associated Legendre function, and $\Sigma$ indicates summation with respect to even indices only. Odd terms do not appear because $\mathrm{l}(\mathrm{y})$ is "symmetric" with respect to the origin at the point P, i.e., $l(\underline{v})=1(-\underline{v})$ or $l(\Phi, \theta)=1(\Phi+\pi, \pi-\theta)$. The desired "best fit" approximation can be established by the "Least Square Method" and leads to 
representation in terms of three-dimensional Cartesian tensor (after Kanatani, in 1984 and 1985; also used by Onat, in 1984 to describe the density of distribution of penny-shaped voids),

$$
n(\underline{v}) \approx n_{0}\left(1+\Omega_{i j} v_{i} v_{j}+\Omega_{i j k l} v_{i} v_{j} v_{k} v_{l}+\ldots\right)
$$

Here, $\Omega_{i j}, \Omega_{i j k l}, \ldots$ are symmetric traceless tensor whose components are combinations of the spherical expansion coefficients $a_{n m}, b_{n m}, m=1,2, \ldots, n$. In particular for $\mathrm{n}=2$, there is,

$$
4 \Omega_{i j}=\left[\begin{array}{ccc}
-a_{20}+12 a_{22} & 12 b_{22} & 6 a_{21} \\
12 b_{22} & -a_{20}-12 a_{22} & 6 b_{21} \\
6 a_{21} & 6 b_{21} & 2 a_{20}
\end{array}\right]
$$

In terms of $n(\underline{v})$ equation(2.12) becomes,

$$
\Omega_{i 1 \cdots i n}=\frac{2 n+1}{2^{n} n_{0}}\left(\begin{array}{c}
2 n \\
n
\end{array}\right) N_{(i 1 \ldots i n\}}
$$

where $\mathrm{N}_{(i 1 \ldots \text { in) }}$ is the n-th "moment" tensor of $\mathbf{L}(\underline{\mathbf{v}})$, i.e., 


$$
N_{(i 1 \ldots i n)}=\int_{S(P)} v_{i 1} \ldots v_{i n} L(\underline{v}) d S(\underline{v})
$$

and \{\} designates the deviatoric part of a symmetric tensor. In particular for $\mathrm{n}=2$, $N_{i j}$ is related to $n_{0}$ and $\Omega_{i j}$ by,

$$
\begin{aligned}
& n_{0}=N_{i j} \\
& \Omega_{i j}=\frac{15}{8 \pi n_{0}} N_{i j}-\frac{5}{2} \delta_{i j}
\end{aligned}
$$

where $\delta_{i j}$ is the Kronecker delta.

For isotropic distribution of pores $\Omega_{\mathrm{ij}}$ vanishes, thus $\Omega_{\mathrm{ij}}$ may be accepted in the "first approximation" as the measure of deviation from isotropy. The components of the higher ranks traceless tensor, i.e., $\Omega_{i j k l}, \Omega_{i j k l} \cdots m$ describe the higher order fluctuations of the void space distribution. The statistical analysis of their influences on the fitness of approximation was discussed by Kanatani (1984). It should be noted that, in view of equation (2.6), the approximation (2.11) directly describes the distribution of the volumetric fraction of voids

$$
V_{v}(\underline{v}) \approx V_{v 0}\left(1+\Omega_{i j} v_{i} v_{j}+\Omega_{i j k l} v_{i} v_{j} v_{k} v_{l}+\ldots\right)
$$


Thus, the same representation can also be used in the context of 'directional void ratio', the latter being defined as

$$
\mathrm{e}(\underline{\mathrm{v}})=\mathrm{V}_{\mathrm{v}}(\underline{\mathrm{v}}) / \mathrm{V}_{\mathrm{s}}
$$

with $\mathrm{V}_{\mathrm{s}}$ representing the volume fraction of solids in $\mathrm{V}^{*}$.

If the approximation of pores distribution is limited to the first two terms of the expansion (2.16), i.e.,

$$
\mathrm{n}(\underline{\mathrm{v}}) \approx \mathrm{n}_{0}\left(1+\Omega_{\mathrm{ij}} \mathrm{v}_{\mathrm{i}} \mathrm{v}_{\mathrm{j}}\right)
$$

and if the eigenvalues of $\underline{\Omega}$ are distinct, then $n(\underline{v})$ can reflect smooth orthogonal anisotropy. The symmetry axes of the orthogonal anisotropy are coincident with the principal axis of $\underline{\Omega}$. If two eigenvalues of $\underline{\Omega}$ are equal then $n(\underline{\mathbf{v}})$ describes the transverse isotropy.

In order to incorporate the proposed measure of internal structure of soil continuum into a phenomenological description, the form of the evolution law of the components of $\underline{\Omega}$ needs to be specified. Assuming that the deformation of the continuum is time-independent it is possible, on the basis of some experiments (cf. Duncan and Seed, 1966; McConnachi, 1974; Attewell and Farmer, 1976), to express the rate of change of $\underline{\Omega}$ as an isotropic tensor-valued function of the rate of strain 
deviator $\underline{\mathbf{e}}$, the current value of $\underline{\mathbf{\Omega}}$ and the average porosity (or void ratio), i.e.,

$$
\dot{\Omega}_{i j}=\dot{\Omega}_{i j}\left(\Omega_{k l}, \dot{e}_{k l}, n_{0}\right)
$$

It should be noted that, the above assumption has some correspondence with a theoretical model, developed by Rice and Tracey (1969), for the growth of a spherical void in a non-hardening plastic solid. The most general form of the linear relationship between tensors $\underline{\dot{\Omega}}$ and $\underline{\dot{e}}$ can be written, after Cowin (1985), as

$$
\begin{aligned}
\dot{\Omega}_{i j} & =a_{1} \delta_{i j} \Omega_{k l} \dot{e}_{k l}+a_{2} \delta_{i j} \Omega_{k q} \Omega_{k l} \dot{e}_{q l} \\
& +a_{3} \Omega_{i j} \Omega_{k l} \dot{e}_{k l}+a_{4}\left(\Omega_{i j} \Omega_{k q} \Omega_{q l}+\Omega_{i s} \Omega_{s j} \Omega_{k l}\right) \dot{e}_{k l}+a_{5} \Omega_{i s} \Omega_{s j} \Omega_{k q} \Omega_{q l} \dot{e}_{k l} \\
& +2 a_{6} \dot{e}_{i j}+2 a_{7}\left(\Omega_{i k} \dot{e}_{k j}+\Omega_{k j} \dot{e}_{k j}\right)+2 a_{8}\left(\Omega_{i r} \Omega_{r k} \dot{e}_{k j}+\Omega_{k r} \Omega_{r j} \dot{e}_{k j}\right)
\end{aligned}
$$

where the coefficients $a_{i}(i=1,8)$ are functions of $n_{0}$ and the three basic invariants of $\underline{\Omega}$, i.e., $\operatorname{tr} \underline{\Omega}, \operatorname{tr} \underline{\Omega}^{2}, \operatorname{tr} \underline{\Omega}^{3}$. The linearization of the dependence of $\underline{\dot{\Omega}}$ on $\underline{\Omega}$ may be obtained by retaining the second order terms $\Omega_{i j}$ in equation (2.20). Such representation, which preserves the orthotropic material symmetry was proposed by Cowin (1985) and takes the form 


$$
\begin{aligned}
\dot{\Omega}_{i j} & =f_{1} \delta_{i j} \Omega_{k l} \dot{e}_{k l}+f_{2} \delta_{i j} \Omega_{k q} \Omega_{q l} \dot{e}_{k l}+f_{3} \Omega_{i j} \Omega_{k l} \dot{e}_{k l} \\
& +f_{4} \dot{e}_{i j}+f_{5}\left(\Omega_{i k} \dot{e}_{k j}+\Omega_{k j} \dot{e}_{k j}\right)+f_{6}\left(\Omega_{i r} \Omega_{r k} \dot{e}_{k j}+\Omega_{r k} \Omega_{r j} \dot{e}_{k i}\right)
\end{aligned}
$$

Here, $f$ 's are function of $n_{0}$ and three basic invariants of $\Omega$, i.e.,

$$
\begin{aligned}
& f_{1}\left(n_{0}, I_{2 \Omega}, I_{3 \Omega}\right)=g_{1}\left(n_{0}\right) \\
& f_{2}\left(n_{0}, I_{2 \Omega}, I_{3 \Omega}\right)=g_{2}\left(n_{0}\right) \\
& f_{3}\left(n_{0}, I_{2 \Omega}, I_{3 \Omega}\right)=g_{3}\left(n_{0}\right) \\
& f_{4}\left(n_{0}, I_{2 \Omega}, I_{3 \Omega}\right)=g_{4}\left(n_{0}\right)+g_{5}\left(n_{0}\right) I_{2 \Omega} \\
& f_{5}\left(n_{0}, I_{2 \Omega}, I_{3 \Omega}\right)=g_{6}\left(n_{0}\right) \\
& f_{6}\left(n_{0}, I_{2 \Omega}, I_{3 \Omega}\right)=g_{7}\left(n_{0}\right)
\end{aligned}
$$

where,

$$
2 I_{2 \Omega}=\Omega_{i k} \Omega_{k i} \text { and } I_{3 \Omega}=\Omega_{i j} \Omega_{j k} \Omega_{k i}
$$

The experimental evidence pertaining to clays indicates that the principal axis of $\underline{\dot{g}}$ might be considered as coaxial with the principle axis of strain rate deviator (cf.Duncan and Seed, 1966; McConnachi, 1974; Delage and Lefebvre, 1984; Kanatani, 1984). The above assumptions leads to a significant simplification of equation (2.21), since all function of $f$ except $f_{4}$ may be set to zero. Thus,

$$
\dot{\Omega}_{i j}=\left[g_{4}\left(n_{0}\right)+g_{5}\left(n_{0}\right) I_{2 \Omega}\right] \dot{e}_{i j}
$$


where functions $g_{4}\left(n_{0}\right)$ and $g_{5}\left(n_{0}\right)$ should reflect the basic experimental trends in the evolution of the soil fabric, i.e.,

- the non-uniform distribution of voids space could not be reached in a very loose soil, i.c., soil domains are apart from one another,

- for a very compact soil structure, the fabric changes reach their saturation level, - nonuniform soil structure, i.e. $I_{20}+0$, has its pronounced effects in a certain range of voids volume.

The constraints mentioned above are satisfied by assuming the following forms for $g_{4}\left(n_{0}\right)$ and $g_{5}\left(n_{0}\right)$ functions

$$
\mathrm{g}_{4}\left(\mathrm{n}_{0}\right)=\mathrm{A}
$$

and,

$$
g_{5}\left(n_{0}\right)=B\left\{1-\exp \left(-I_{n}\right)\right\}
$$

where $A$ and $B$ are the material constants and $I_{n}$ represents the porosity index defined by

$$
I_{n}=\frac{n_{0}-n_{s}}{n_{L}-n_{s}}
$$

Here, $n_{L}$ and $n_{s}$ are porosities corresponding to the liquid and the shrinkage limits, respectively. 
23 Sample estimation of porosity tensor based on available microscopic evidence.

If the approximation of voids distribution is confined to the representation (2.18)

$$
n(\underline{v}) \approx n_{0}\left(1+\Omega_{i j} v_{i} v_{j}\right)
$$

in which spherical harmonics of degree higher than two are disregarded, and if, according to equation (2.7a)

$$
\mathrm{n}(\underline{\mathbf{v}})=\mathrm{L}(\underline{\mathrm{v}})
$$

then, the components of tensor $\underline{\Omega}$ can be estimated from the observation of $L(\underline{y})$ on a limited number of cross-sections. In what follows, the procedure for the stereological estimation of the directional porosity distribution, given by equation (2.18), is outlined (after general recommendations given by Kanatani, 1985). The presented sample calculations are completed by using the results of an experimental study of the structure of the Champlain clay and its evolution during a standard one dimensional consolidation, as reported by Delage and Lefebvre (1984).

Consider a cross-section of soil sample in a plane $\pi(\underline{u})$ with unit normal $\underline{\underline{u}}=\left\{\mathrm{u}_{\mathfrak{v}}, \mathrm{u}_{2}, \mathrm{u}_{3}\right\}^{\top}$, with respect to an orthogonal Cartesian coordinate system fixed 
in the material. Place a bunch of sampling lines laying in the plane $\pi(\underline{\underline{u}})$, having directions $\underline{\mathbf{v}}$ and emanating from a common point $\mathrm{P}$, being the point of intersection of a cross-sectional plane with a line $U(\underline{u})$ orthogonal to the plane $\pi(\underline{u})$. Then, the 'zero' moment and the 'second' skew-symmetric moment of quantity $L(\underline{y})$ in the plane $\pi(\underline{u})$ with regard to a pole $\mathrm{P}$ are,

$$
\begin{aligned}
& M(\underline{u})=\int_{C(\underline{u})} L(\underline{v}) d s(\underline{v}) \\
& M_{i j}(\underline{u})=\int_{C(\underline{u})} v_{i} v_{j} L(\underline{v}) d s(\underline{v})
\end{aligned}
$$

Here, $\mathrm{C}(\underline{\underline{u}})$ is the unit circle rounding the $\mathrm{U}(\underline{\mathrm{u}})$ line perpendicularly and $\mathrm{ds}(\underline{\mathbf{v}})$ is the arc length measure of $C(\underline{u})$ normalized with respect to $2 \pi$, as sketch schematically in Fig. 2.2. Substituting the approximations given by equations (2.18) and (2.7a) in equations (2.26) and (2.27) the moments of $L(\underline{v})$ can be expressed in terms of $\Omega_{i j}$ (cf. Kanatani, 1985),

$$
\begin{aligned}
& M(\underline{u})=2 \pi n_{0}\left(1-\frac{1}{2} \Omega_{i j} v_{i} v_{j}\right) \\
& M_{i j}(\underline{u})=\pi n_{0}\left[\frac{1}{2} \Omega_{i j}-\frac{3}{2} \Omega_{k(i} v_{j} v_{k}+\left(1-\frac{1}{4} \Omega_{k l} v_{k} v_{l}\right) v_{i} v_{j}\right]
\end{aligned}
$$

where parentheses ( ) desigaate the symmetrized indices. 
Assume that the experimental observations are made on three perpendicular planes with unit normals parallel to the base vectors $\underline{\mathbf{e}}_{1}=\{1,0,0\}^{\top}, \underline{e}_{2}=\{0,1,0\}^{\top}, \underline{e}_{3}=\{0,0,1\}^{\top}$. In this case, the conponents of tensor $\underline{\Omega}$ are given by the following expressions,

$$
\begin{aligned}
& \Omega_{11}=2-\frac{M\left(\underline{e}_{1}\right)}{\pi n_{0}} \\
& \Omega_{22}=2-\frac{M\left(\underline{e}_{2}\right)}{\pi n_{0}} \\
& \Omega_{33}=2-\frac{M\left(\underline{e}_{3}\right)}{\pi n_{0}} \\
& \Omega_{12}=2-\frac{2 M_{12}\left(\underline{e}_{3}\right)}{\pi n_{0}} \\
& \Omega_{23}=2-\frac{2 M_{23}\left(\underline{e}_{1}\right)}{\pi n_{0}} \\
& \Omega_{31}=2-\frac{2 M_{31}\left(\underline{e}_{2}\right)}{\pi n_{0}}
\end{aligned}
$$

According to equations (2.30) and the definition (2.26) the diagonal terms of $\underline{\Omega}$ arc proportional to average porosities measured on planes perpendicular to base vectors, whereas, the off-diagonal terms describe the deviation of principal directions of $\underline{\Omega}$ relative to the referential coordinate system. The integrals $M\left(\underline{e}_{k}\right)$ and $M_{i j}\left(\underline{e}_{k}\right)$ can be calculated using the trapezoidal integration scheme 


$$
\begin{aligned}
& M\left(\underline{e}_{k}\right)=2 \pi \sum_{m=0}^{N-1} L\left(\underline{e}_{k}, \alpha_{m}\right) / N \\
& M_{i j}\left(\underline{e}_{k}\right)=\pi \sum_{m=0}^{N-1} L\left(\underline{e}_{k}, \alpha_{m}\right) \sin (2 \pi m / N) / N
\end{aligned}
$$

Here, $L\left(\underline{\boldsymbol{c}}_{\mathbf{k}}, \alpha_{\mathrm{m}}\right)$ is the fraction of the test line (occupied by voids), whose orientation, in a plane with unit normal $\underline{\mathbf{e}}_{\mathbf{k}}$, is described in terms of an angle $\alpha$, i.e.

$$
\alpha_{m}=m \pi / N(m=0,1,2, \ldots, N-1) \text {. }
$$

The above estimates for the components of $\underline{\Omega}$, in terms of the moments of L(v) were employed to evaluate the porosity distribution and its evolution induced by $\mathrm{K}_{\mathrm{o}}$-consolidation process. In particular, the experimental data reported by Delage and Lefebvre (1984) were used. According to Delage and Lefebvre (1984), the undisturbed samples of the Champlain clay were consolidated in a standard oedometer with 1.5 load increment ratio applied every $24 \mathrm{~h}$ to $23 \mathrm{kPa}, 124 \mathrm{kPa}$, $421 \mathrm{kPa}$ and $1452 \mathrm{kPa}$, respectively. The load was then released in several stages and samples were left $24 \mathrm{~h}$ under the load of $4 \mathrm{kPa}$. Specimens, for microscopic structure identification, were trimmed in a form of small sticks with a thin wire, quick frozen in Freon 22, cooled by liquid nitrogen, freeze-fractured, and sublimated. The observations of the clay cross-sections were performed using JEOL 25 scanning electron microscope. A graphical representations of porosity, in a horizontai and a vertical plane, at two different levels of the consolidation process, are presented in Figure 2.3, after Delage and Lefebvre, 1984. 
For a purpose of lineal analysis a discus part of the cross-sections presented in Fig. 2.3 was considered. The set of eighteen probe lines was drawn $(\mathrm{N}=18)$, with orientations $\alpha_{m}=(m-1) \pi / N(m=1, \ldots, 18)$, as sketched in Fig. 2.4. Then, the total length of the intersections of each test line with pores was measured and, subsequently, the length of the intersections per unit length of the test line $L\left(e_{k}, \alpha_{m}\right)$ was evaluated. The results of measurements and calculations are summarized in Table 2.1 for the intact sample, and in Table 2.2 for the sample consolidated under the pressure $421 \mathrm{kPa}$.

During the one dimensional consolidation, both under in situ conditions and in oedometer, an axisymmetric deformation mode is imposed. Therefore, the evolution of the porosity distribution was restricted to one vertical plane only (Delage and Lefebvre. 1984). In calculating the components of tensor $\underline{\Omega}$ it was assumed that,

$$
M\left(\underline{e}_{2}\right)=M\left(\underline{e}_{1}\right) \text { and } M_{13}\left(\underline{e}_{2}\right)=M_{23}\left(\underline{e}_{1}\right)
$$


'Table 2.1. Calculations of porosity distribution for the intact sample

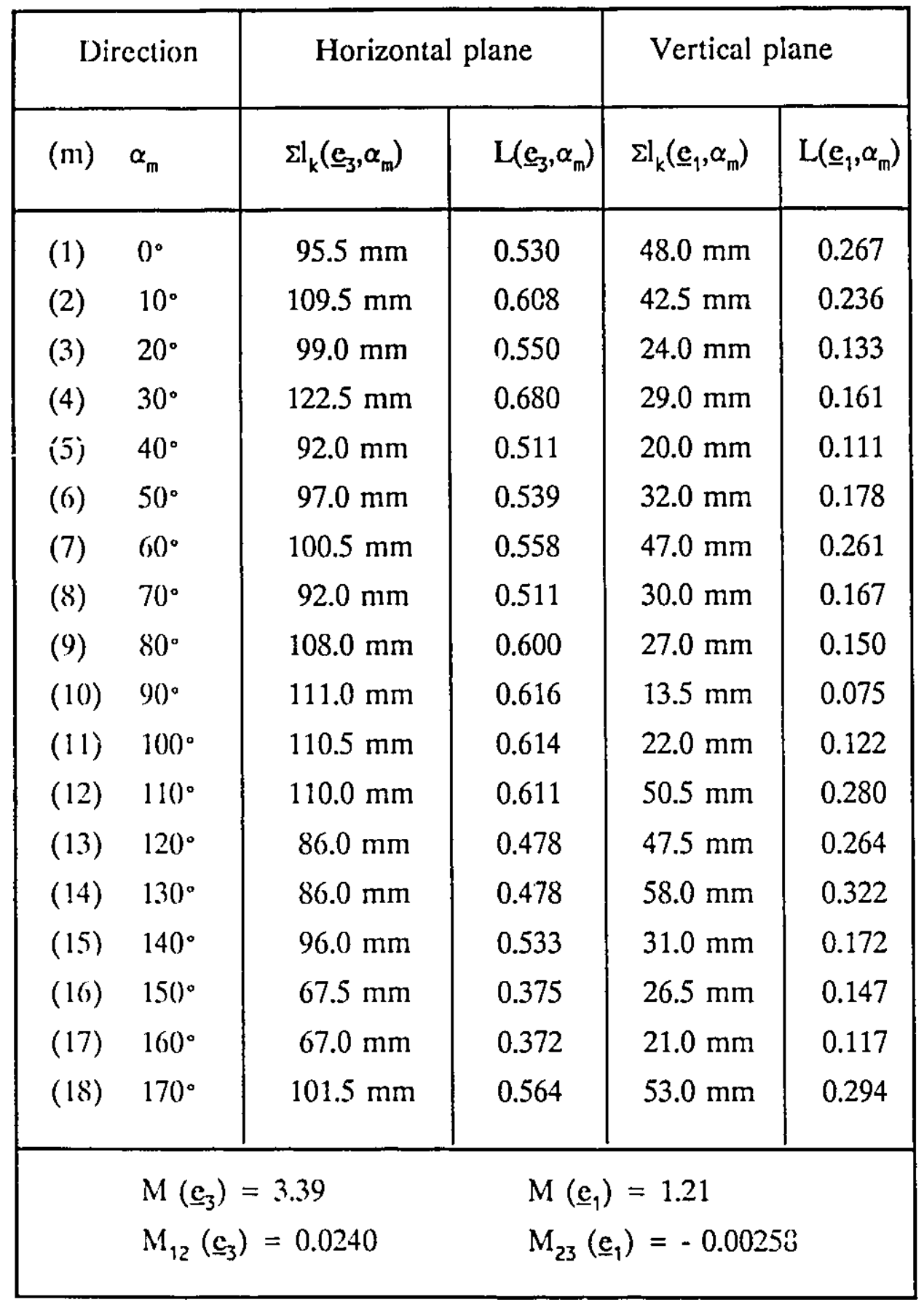


Table 2.2. Calculations of porosity distribution for the consolidated simmple

\begin{tabular}{|c|c|c|c|c|c|}
\hline \multicolumn{2}{|c|}{ Direction } & \multicolumn{2}{|c|}{ Horizontal plane } & \multicolumn{2}{|c|}{ Vertical plane } \\
\hline (m) & $\alpha_{m}$ & $\Sigma l_{k}\left(\underline{e}_{3}, \alpha_{m}\right)$ & $\mathbf{L}\left(\underline{\mathbf{e}}_{3}, \alpha_{\mathrm{m}}\right)$ & $\Sigma l_{k}\left(\underline{c}_{1}, \alpha_{m}\right)$ & $\mathrm{L}\left(\underline{\mathrm{c}}_{1}, \alpha_{\mathrm{m}}\right)$ \\
\hline (1) & $0^{\circ}$ & $47.2 \mathrm{~mm}$ & 0.262 & $50.0 \mathrm{~mm}$ & 0.278 \\
\hline (2) & $10^{\circ}$ & $55.0 \mathrm{~mm}$ & 0.305 & $33.0 \mathrm{~mm}$ & 0.183 \\
\hline (3) & $20^{\circ}$ & $50.0 \mathrm{~mm}$ & 0.228 & $43.5 \mathrm{~mm}$ & 0.242 \\
\hline (4) & $30^{\circ}$ & $58.5 \mathrm{~mm}$ & 0.325 & $36.0 \mathrm{~mm}$ & 0.200 \\
\hline$(5)$ & $40^{\circ}$ & $33.0 \mathrm{~mm}$ & 0.183 & $28.5 \mathrm{~mm}$ & 0.158 \\
\hline$(6)$ & $50^{\circ}$ & $40.0 \mathrm{~mm}$ & 0.222 & $30.0 \mathrm{~mm}$ & 0.167 \\
\hline (7) & $60^{\circ}$ & $55.5 \mathrm{~mm}$ & 0.308 & $22.0 \mathrm{~mm}$ & 0.122 \\
\hline$(8)$ & $70^{\circ}$ & $94.0 \mathrm{~mm}$ & 0.522 & $15.0 \mathrm{~mm}$ & 0.083 \\
\hline (9) & $80^{\circ}$ & $53.0 \mathrm{~mm}$ & 0.294 & $12.5 \mathrm{~mm}$ & 0.069 \\
\hline$(10)$ & $90^{\circ}$ & $33.0 \mathrm{~mm}$ & 0.183 & $4.0 \mathrm{~mm}$ & 0.022 \\
\hline (11) & $100^{\circ}$ & $0.0 \mathrm{~mm}$ & 0.0 & $7.0 \mathrm{~mm}$ & 0.039 \\
\hline$(12)$ & $110^{\circ}$ & $12.0 \mathrm{~mm}$ & 0.067 & $5.0 \mathrm{~mm}$ & 0.278 \\
\hline (13) & $120^{\circ}$ & $14.0 \mathrm{~mm}$ & 0.078 & $29.0 \mathrm{~mm}$ & 0.161 \\
\hline$(14)$ & $130^{\circ}$ & $29.5 \mathrm{~mm}$ & 0.164 & $23.0 \mathrm{~mm}$ & 0.128 \\
\hline$(15)$ & $140^{\circ}$ & $27.0 \mathrm{~mm}$ & 0.150 & $23.5 \mathrm{~mm}$ & 0.130 \\
\hline$(16)$ & $150^{\circ}$ & $57.5 \mathrm{~mm}$ & 0.319 & $15.5 \mathrm{~mm}$ & 0.086 \\
\hline$(17)$ & $160^{\circ}$ & $35.0 \mathrm{~mm}$ & 0.194 & $30.0 \mathrm{~mm}$ & 0.167 \\
\hline (18) & $170^{\circ}$ & $51.2 \mathrm{~mm}$ & 0.286 & $29.5 \mathrm{~mm}$ & 0.164 \\
\hline & $\begin{array}{l}M\left(\underline{c}_{3}\right) \\
M_{12}(\underline{c}\end{array}$ & $\begin{array}{l}1.45 \\
=0.0416\end{array}$ & $M_{23}$ & $\begin{array}{l}=0.847 \\
\left.{ }_{1}\right)=-0.002\end{array}$ & \\
\hline
\end{tabular}


The results, using expressions (2.33a) to (2.33f) and values given in Table 2.1 and Table 2.2., are presented below.

\section{Intact sample}

$$
\begin{array}{ll}
n_{0}=0.308, & e_{0}=0.445 \\
\Omega_{11}=0.745, & \Omega_{22}=0.745, \quad \Omega_{33}=-1.508 \\
\Omega_{12}=0.0, & \Omega_{23}=-0.00532, \quad \Omega_{13}=-0.00532
\end{array}
$$

the principal values of $\Omega_{i j}$ are,

$$
\Omega_{11}=0.754, \Omega_{22}=0.754, \Omega_{33}=-1.508, \theta=0^{\circ}
$$

Consolidated sample

$$
\begin{array}{ll}
\mathrm{n}_{0}=0.167, & \mathrm{e}_{0}=0.200 \\
\Omega_{11}=0.381, & \Omega_{22}=0.381, \Omega_{33}=-0.762 \\
\Omega_{12}=0.0, & \Omega_{23}=0.00858, \Omega_{13}=0.00858
\end{array}
$$

the principal values are,

$$
\Omega_{11}=0.381, \Omega_{22}=0.381 \quad, \Omega_{33}=-0.762, \quad \theta=0^{\circ}
$$


The porosity profiles, for both intact and consolidated sample, are presented in graphical form in Fig. 2.5. It should be noted that the average porosities $n_{0}$, as estimated by the described stereological procedure, are in agreement with values reported by Delage and Lefebvre (1984). 


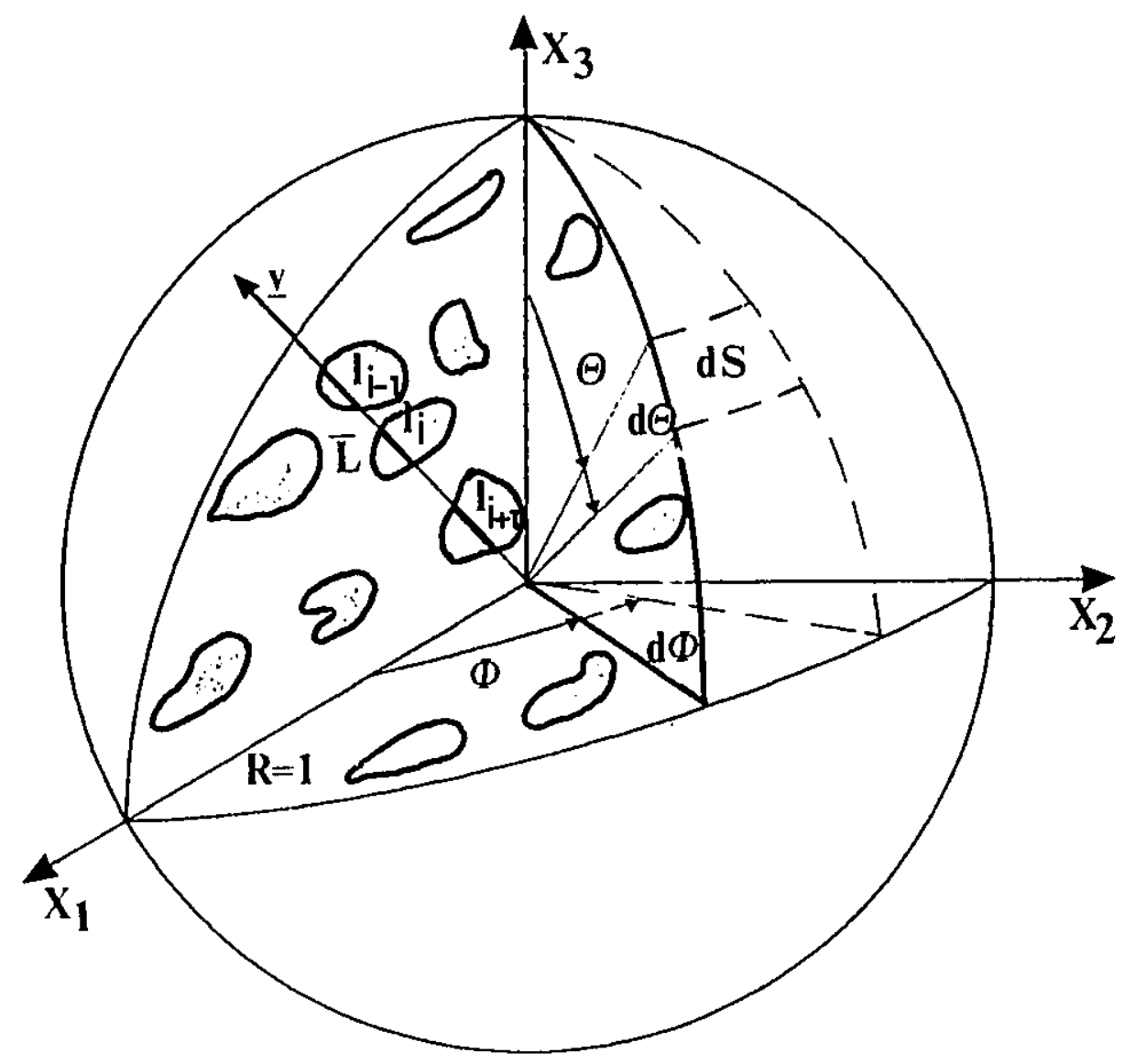

Figure 2.1 Unit sphere enclosing a representative volume of the material.

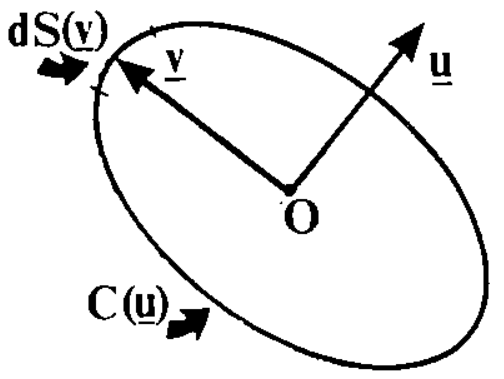

Figure 2.2 Unit vector $y$ of a circle and unit normal vector $\underline{u}$ to the plane of the circle. 
Vertical planc

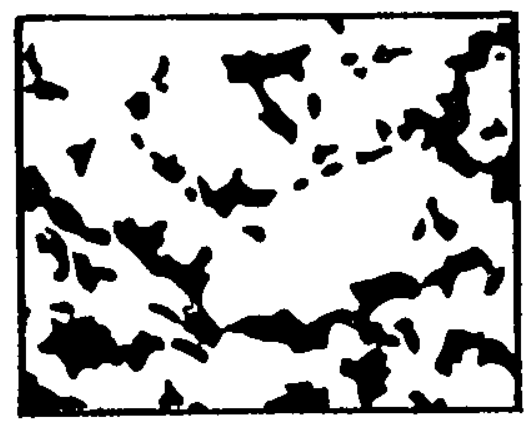

I n t act

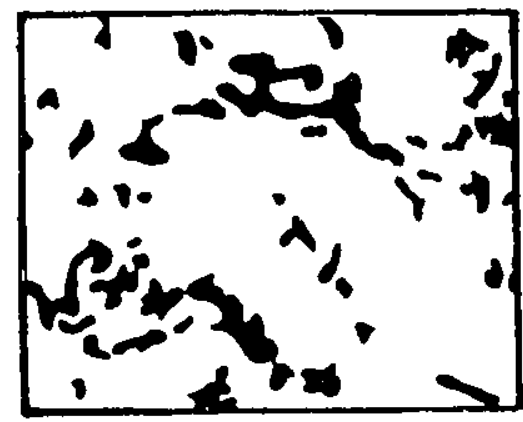

$421 \mathrm{kPa}$

Horizontal plane

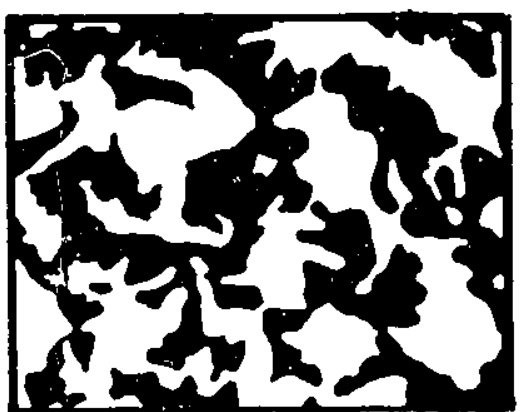

In t a ct

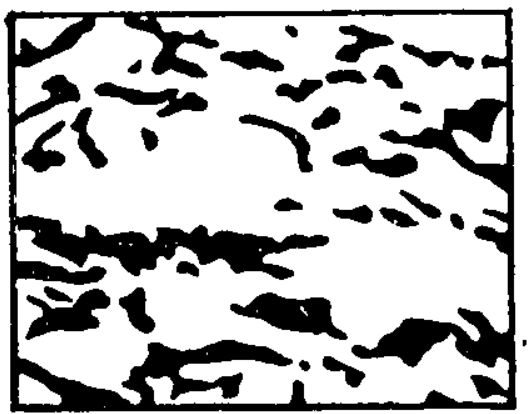

$421 \mathrm{kPa}$

Figure 2.3 Graphical representation of porosity, at. diffrent stages of consolidation of Champlain clay (after Delage and Lefebvre, 1984); voids are black and particles are white. 


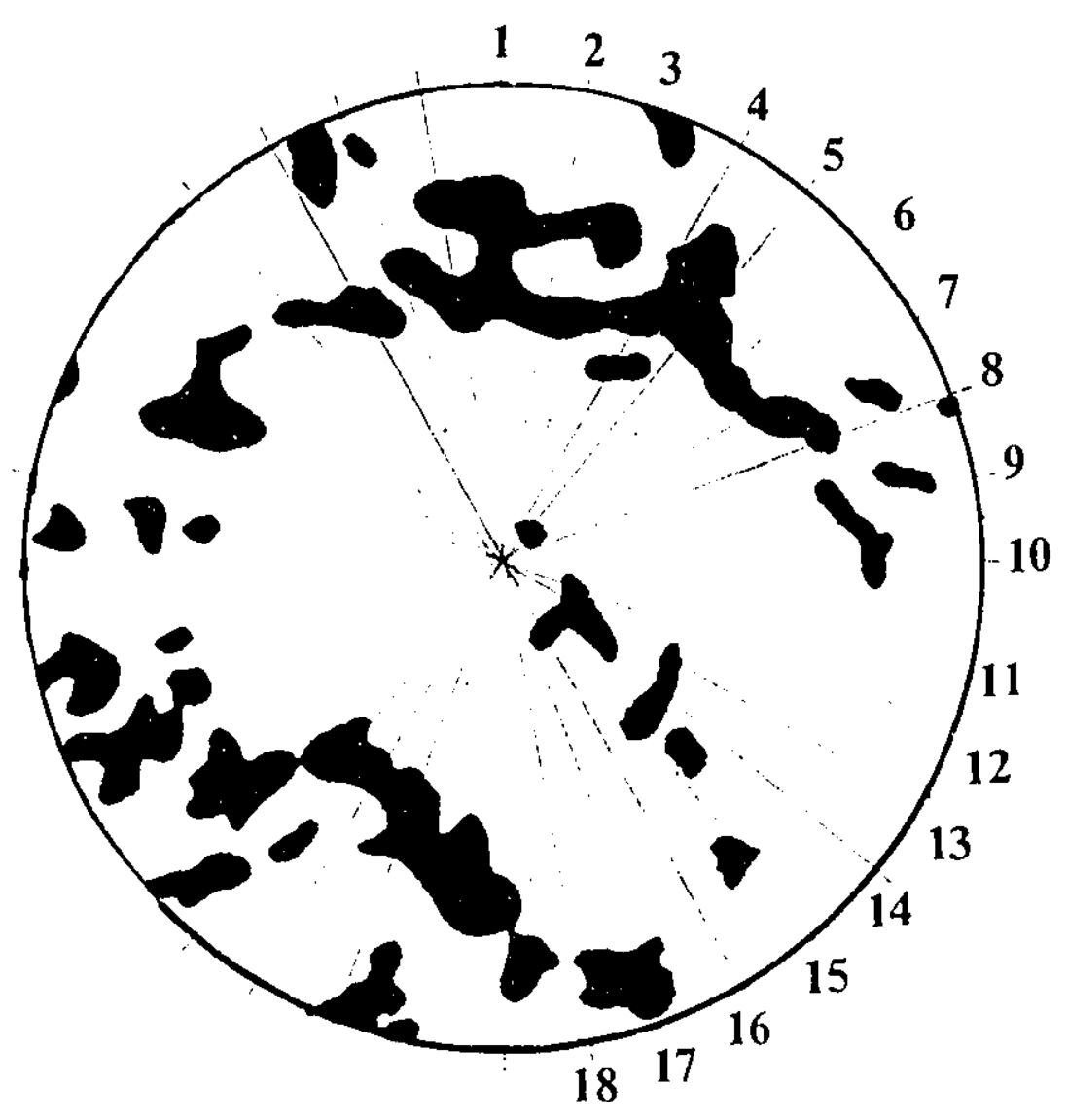

Figure $2.4 \Lambda$ discus part of the cross-scction, from Figure, 2.3, subtracted for a purpose of lineal analysis. 


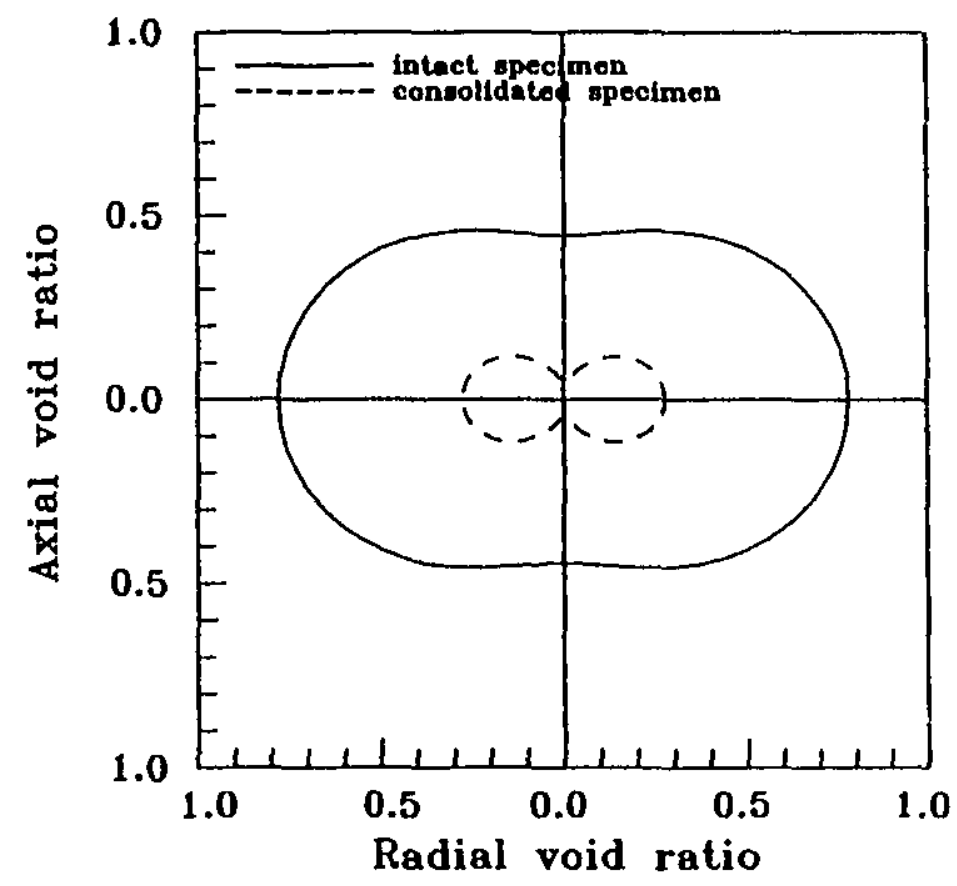

Figure 2.5 Spatial distribution of void ratio for intact and consolidated sample of Champlain Clay. 


\section{CHAPTER 3}

\section{PLASTICITY FORMULATION FOR ANISOTROPIC PARTICULATE MEDIA}

\subsection{Introduction; yield criteria for anisotropic media.}

Historically, the first attempt to introduce the dependence of plastic flow on inherent anisotropy was made by Hill (1950). He suggested that anisotropic deformation of a metal sheet, where orthotropic symmetry is expected (with symmetry axis perpendicular to the plane of the sheet, and parallel and perpendicular to the ro!!ing direction), can be described by an associated flow rule with a yield function in the form of a generalized von Misses yield criterion, i.e.,

$$
\begin{aligned}
2 \mathrm{f}\left(\sigma_{\mathrm{ij}}\right)= & \mathrm{F}\left(\sigma_{22}-\sigma_{33}\right)^{2}+\mathrm{G}\left(\sigma_{33}-\sigma_{11}\right)^{2}+\mathrm{H}\left(\sigma_{11}-\sigma_{22}\right)^{2}+ \\
& 2 \mathrm{~L}\left(\sigma_{32}\right)^{2}+2 \mathrm{M}\left(\sigma_{31}\right)^{2}+2 \mathrm{~N}\left(\sigma_{12}\right)^{2}=1
\end{aligned}
$$

In expression (3.1) the stress tensor $\underline{\sigma}$ is referred to the principal axes of anisotropy and $F, G, H, L, M, N$ are material constants, whose values can be determined from pure shear and simple tension experiments (Johnson, Sowerby and Ventor, 
1982). A frame-indifferent generalization of the yield function (3.1) can be written as follows

$$
f=C_{i j k l} s_{i j} s_{k l}-2 k^{2}=0
$$

Here, a fourth order tensor $C_{i j k l}$ characterizes material symmetry and $k$ is the yield stress in pure shear. In the case of isotropy $C_{i j k l}$ is identified with a fourth order isotropic tensor, i.e.,

$$
C_{i j k l}=c_{1} \delta_{i j} \delta_{k l}+c_{2} \delta_{i k} \delta_{j l}+c_{3} \delta_{i l} \delta_{j k}
$$

When, the scalar coefficients $c_{i}(i=1,2,3)$ are selected as

$$
c_{1}=1, c_{2}=c_{3}=\frac{1}{2} .
$$

equation (3.2) reduces to the von Misses yield criterion, i.e.,

$$
f=s_{i j} s_{i j}-2 k^{2}=0
$$

If some specific constrains are imposed on the coupling between stress and strain tensor (cf. Johnson, Sowerby and Ventor, 1982), then for a case of an orthotropic incompressible material the number of the independent components of $C_{i j k l}$ can be 
reduced to six. In addition, these six independent components can be related to the empirical constants appearing in equation (3.1), as demonstrated by Sowerby (1988).

A generalized version of Hill's model, accounting for an evolution of the anisotropy tensor $\mathrm{C}_{\mathrm{ijkl}}$ with progressing deformation, found certain practical applications in the analysis of a rolled-sheet forming process (Johnson, Sowerby and Ventor, 1982). However, equation (3.2) can only describe an isotropic hardening process. Thus, Hill's formulation is not capable of modelling such important phenomena as the Bauschinger effect and induced anisotropy. One way of describing these effects is by means of a plasticity formulation incorporating a kinematic hardening. Several such formulations have aiready been proposed in the literature (e.g. Prevost, 1978; Pietruszczak and Mroz, 1983; Anandarajah and Dafalias, 1986). An alternative formulation may be derived by employing a more general concept of the anisotropic hardening rule, as primarily proposed for pressure non-sensitive materials by Shrivastava, Mroz and Dubey (1973) or, recently defined in a more versatile form by Bohler (1987). Following the discussior presented by Shrivastava, Mroz and Dubey (1973), the yield condition can be assumed in the following form

$$
f=f\left(s_{i j}, \alpha_{i j}, x\right)=0
$$


where $\underline{\alpha}$ denotes a tensorial hardening parameter, $æ$ is a scalar function tracing history of irreversible deformation, and tensor $\mathbf{s}$ defines the stress deviator. Employing theorems of representation for a scalar-valued function of a tensor, the yield criterion (3.4) can be represented by a polynomial in the integrity basis of tensors $\underline{\mathbf{s}}$ and $\underline{\boldsymbol{\alpha}}$. Further assumptions concerning the specification of the coefficients entering the polynomial expansion of equation (3.4), results in the following tensorial form of an anisotropic yield criterion (cf. Shrivastava, Mroz and Dubey, 1973)

$$
f=C_{i j k l}\left(\sigma_{i j}-\alpha_{i j}\right)\left(\sigma_{k l}-\alpha_{k l}\right)+p\left(\operatorname{trs}^{3}\right)-k^{2}(x)=0
$$

where,

$$
\begin{aligned}
& C_{i j k l}=\left(I_{i j k l}+A \alpha_{i j k l} \alpha_{k l}\right) \\
& I_{i j k l}=\frac{3}{2}\left(\delta_{i k} \delta_{j l}+\delta_{i t} \delta_{j k}\right)
\end{aligned}
$$

and $\mathrm{A}$ and $\mathrm{p}$ are material constants. In geometrical terms the surface described by equation (3.5) can deform, rotate as well as translate in stress space owing to the evolution of the tensorial hardening parameter $\underline{\alpha}$. In particular, if the components of $\underline{\underline{\alpha}}$ are assumed to be proportional to the plastic strain tensor, i.e., 


$$
\alpha_{i j}=c \epsilon_{i j}^{p} ; \quad c=\text { const. }
$$

then some of the formerly proposed anisotropic yield criteria for plastic solid (e.g. Ziegler, 1959; Baltov and Sawczuk, 1965) can be obtained, as extensively discussed by Shrivastava, Mroz and Dubey (1973).

A systematic generalization of the various hardening rules for anisotropic plastic solids can be obtained by relating the description of hardening process to the isotropic or anisotropic transformation of stress tensor. Such a broad and consistent concept was proposed by Bohler (1987) and is shortly outlined below. Let the functional form of an anisotropic yield function, defined in stress space, be identified with a scalar function of tensor arguments $\underline{\sigma}, \underline{\alpha}^{(i)}, \underline{\epsilon}^{\mathfrak{p}}$, i.e.,

$$
f=f\left(\sigma_{i j}, \alpha_{i j}{ }^{(i)}, \epsilon_{i j}{ }^{p}\right)
$$

According to the Cayley-Hamilton Theorem one can write the function (3.6a) in terms of invariants of tensors $\underline{\sigma}, \underline{\alpha}^{(i)}, \underline{\epsilon}^{\mathrm{p}}$, i.e.,

$$
\mathrm{f}=\mathrm{f}\left(\mathrm{I}_{10}, \mathrm{I}_{2 v}, \mathrm{I}_{3 v}, \mathrm{~J}_{\mathrm{k}}\right)=0
$$

Here, $\mathrm{I}_{1 v}, \mathrm{I}_{2 v}, \mathrm{I}_{3 v}$ are invariants $c \quad \alpha$ sensor $\underline{\sigma}$ and $\mathrm{J}_{\mathrm{k}}$ stands for an irreducible set of mixed invariants of $\underline{\sigma}, \underline{\alpha} \quad$. . Once again $\underline{\alpha}^{(i)}$ denotes a set of tensors 
representing material symmetry. Ultimately, a general concept of the hardening rule is based on the assumption that the evolution of function (3.6) with progressing deformation is taken into account by the specific transformation of stress tensor (cf. Bohler, 1987), i.e.,

$$
\underline{\bar{\sigma}}=\underline{\bar{\sigma}}\left(\sigma_{i j}, \underline{\alpha}_{i j}{ }^{(i)}, \underline{\epsilon}^{\mathfrak{p}}\right)
$$

The transformation indicated by equation (3.7) modifies the yield function (3.6) to the form

$$
\mathrm{f}=\mathrm{f}\left(\mathrm{I}_{1 \bar{v}}, \mathrm{I}_{2 \vec{\sigma}}, \mathrm{I}_{3 \bar{\sigma}}, \overline{\mathrm{J}}_{\mathrm{k}}\right)=0
$$

The effects of both inherent and induced anisotropy as well as coupling between them, are embodied by the mixed invariants $\bar{J}_{k}$ of tensors $\underline{\sigma}, \underline{\alpha}^{(i)}$ and $\underline{\epsilon}^{\mathfrak{p}}$. Therefore, in view of equations (3.6) to (3.8) the modelling of anisotropy is reduced to the specification of stress tensor transformation (3.7). Examples of a such transformation for a number of selected anisotropic yield functions are given by Bohler (1987).

The main deficiency of most of the existing formulations is the formal dependence of the anisotropic hardening function $\underline{\alpha}$ on the plastic strain tensor $\underline{\epsilon}^{p}$, without any correlation to the material structure. In what follows, an alternative 
description will be proposed, incorporating the scalar valued function $\mathrm{n}(\mathbf{y})$ which defines, through the components of $\Omega$, the spatial distribution of porosity/void ratio within the sample.

\subsection{Plasticity framework for materials with inherently anisotropic filbric.}

The spatial distribution of porosity/void ratio can be accepted as an implicit mcasure of internal structure of the material. Obviously, the components of $\Omega$ cannot be strictly identified with the fabric tensor, since the latter is supposed to describe directly the geometry of microstructure, i.e., size and geometrical arrangement of particles. However, one can define the fabric tensor $\underline{\alpha}$ as an isotropic tensor-valued function of tensor $\underline{\Omega}$. The function $\underline{\alpha}=\underline{\alpha}(\underline{\Omega})$ has a polynomial representation of the form

$$
\alpha_{i j}=a_{0} \delta_{i j}+a_{1} \Omega_{i j}+a_{2} \Omega_{i k} \Omega_{k j}
$$

where $a_{0}, a_{1}$ and $a_{2}$ are scalar functions of the basic invariants of $\underline{\Omega}$. The tensor $\underline{\alpha}$ is a symmetric second order tensor which describes, by means of the vectorial form, $\alpha_{i j} v_{j}$ a fabric ellipsoid. The ellipsoid with three unequal axes (associated with the 
material orthotropy) corresponds to three distinct eigenvalues of $\underline{\alpha}$, whereas three equal eigenvalues describe a sphere associated with the isotropy of fabric. It should be noted that when $a_{0}=a_{1}=$ const. and $a_{2}=0$ the representation (3.1) reduces to

$$
\alpha_{i j} \sim \delta_{i j}+\Omega_{i j}
$$

In this case the directional distribution of porosity/void ratio describes by itself the geometric anisotropy in the fabric of the material.

If the local geometric arrangement of the microstructure of porous media displays a significant bias in the distribution, this will inevitably affect the elastic properties of the material as well as the functional form of the failure/yield locus. Assume that in the elastic range the stress tensor is an isotropic tensor-valued function of both strain tensor $\underline{\epsilon}$ and the fabric tensor $\underline{\alpha}$

$$
\sigma_{i j}=\sigma_{i j}\left(\epsilon_{k l}, \alpha_{k l}, n_{0}\right)
$$

The most general representation of the elasticity tensor $D_{i j k l}$ in terms of the fabric tensor, consistent with the isotropy requirement imposed on the function (3.13), has been derived by Cowin (1985). The form is analogous to representation (2.20), i.e., 


$$
\begin{aligned}
D_{i j k l} & =a_{1} \delta_{i j} \delta_{k l}+a_{2}\left(\alpha_{i j} \delta_{k l}+\alpha_{k l} \delta_{i j}\right)+a_{3}\left(\alpha_{k q} \alpha_{q l} \delta_{i j}+\alpha_{i q} \alpha_{q j} \delta_{k l}\right) \\
& +a_{4} \alpha_{i j} \alpha_{k l}+a_{5}\left(\alpha_{i j} \alpha_{k q} \alpha_{q l}+\alpha_{i s} \alpha_{s j} \alpha_{k l}\right)+a_{6} \alpha_{i s} \alpha_{s j} \alpha_{k q q} \alpha_{q l} \\
& +a_{7}\left(\delta_{k i} \delta_{l j}+\delta_{l i} \delta_{k j}\right)+a_{8}\left(\alpha_{i k} \delta_{l j}+\alpha_{k j} \delta_{l i}+\alpha_{i l} \delta_{k j}+\alpha_{l j} \delta_{k i}\right) \\
& +a_{9}\left(\alpha_{i m} \alpha_{m k} \delta_{l j}+\alpha_{k m} \alpha_{m j} \delta_{l i}+\alpha_{i m} \alpha_{m l} \delta_{k j}+\alpha_{l m} \alpha_{m j} \delta_{i k}\right)
\end{aligned}
$$

It has been shown (Cowin, 1985) that orthotropy is the least material symmetry described by the representation (3.14) and it corresponds to the case when the eigenvalues of $\underline{\alpha}$ are distinct. Transverse isotropy is described by two distinct eigenvalues of $\underline{\alpha}$ and the case when components $\alpha_{i j}$ are proportional to $\delta_{i j}$ represents material isotropy. The coefficients $a_{0}$ through $a_{9}$ are functions of the three basic invariants of $\underline{\alpha}$ and their specification has been explained in detail by Cowin (1985).

In order to specify the form of the failure criterion in the presence of fabric anisotropy, one should write

$$
F\left(\sigma_{i j}, \alpha_{i j}\right)=\text { const. }
$$


Since $F$ must be an isotropic function of $\underline{\alpha}$ and $\underline{\sigma}$, it can only depend on ten functionally independent invariants of both tensors. Thus,

$$
F\left\{\operatorname{tr} \underline{\alpha}, \operatorname{tr} \underline{\sigma}^{2}, \operatorname{tr} \underline{\alpha}^{3}, \operatorname{tr} \underline{\sigma}, \operatorname{tr} \underline{\sigma}^{2}, \operatorname{tr} \underline{\alpha}^{3}, \operatorname{tr} \underline{\alpha \sigma}, \operatorname{tr}\left(\underline{\alpha \sigma}^{2}\right), \operatorname{tr}\left(\underline{\sigma \alpha}^{2}\right), \operatorname{tr}\left(\underline{\sigma}^{2} \underline{\alpha}^{2}\right)\right\}=\text { const. }
$$

where

$$
\begin{aligned}
& \operatorname{tr} \underline{\underline{\alpha}}=\alpha_{i i} \quad \operatorname{tr} \underline{\sigma}=\sigma_{i j} \quad \operatorname{tr} \underline{\alpha \sigma}=\alpha_{i k} \sigma_{k i} \\
& \operatorname{tr} \underline{\alpha}^{2}=\alpha_{i k} \alpha_{k i} \quad \operatorname{tr} \underline{\sigma}^{2}=\sigma_{i k} \sigma_{k i} \quad \operatorname{tr}\left(\underline{\alpha \sigma}{ }^{2}\right)=\alpha_{i j} \sigma_{j k} \sigma_{k i} \\
& \operatorname{tr} \underline{\alpha}^{3}=\alpha_{i j} \alpha_{j k} \alpha_{k i} \quad \operatorname{tr} \underline{\sigma}^{3}=\sigma_{i j} \sigma_{j k} \sigma_{k i} \operatorname{tr}\left(\underline{\sigma \alpha}^{2}\right)=\sigma_{i j} \alpha_{j k} \alpha_{k i} \\
& \operatorname{tr}\left(\underline{\sigma}^{2} \underline{\alpha}^{2}\right)=\sigma_{i j} \sigma_{j k} \alpha_{k l} \alpha_{l i}
\end{aligned}
$$

The proper representation can be obtained by expanding (3.16) in a polynomial of its components, i.e. the above basic and simultaneous invariants of $\underline{\alpha}$ and $\underline{\sigma}$. Cowin (1986) developed a quadratic approximation by retaining only linear terms and terms of order two, which leads to

$$
F=A_{i j} \sigma_{i j}+F_{i j k l} \sigma_{i j} \sigma_{k l}=\text { const. }
$$


where

$$
A_{i j}=g_{1} \delta_{i j}+g_{2} \alpha_{i j}+g_{3} \alpha_{i k} \alpha_{k j}
$$

and the representation for $F_{i j k l}$ in terms of $\alpha_{i j}$ is identical with the representation for $D_{i j k l}$ in terms of $\alpha_{i j}$, as given by equation (3.14). The identification of function (3.18) reduces to determination of coefficients $g_{1}, g_{2}, g_{3}$ and $a_{1}$ through $a_{9}$ which are function of the basic invariants of $\underline{\alpha}$. Obviously, other approximations could be employed, as discussed for example by Rowlands (1985).

By inspecting representation (3.11), it appears that an approximation employing $a_{0}=1$ and $a_{2}=0$, i.e.

$$
\alpha_{i j}=\delta_{i j}+a_{1} \Omega_{i j}
$$

may be a reasonable compromise between the accuracy and the complexity of the description of fabric. In samples with inherent isotropy the components of $\underline{\Omega}$ are of the order of accumulated deviatoric strain, as implied by equation (2.24). Therefore, it seems reasonable to speculate that in most cases the deformation process will have a negligible effect on material fabric, i.e.

$$
a_{1}\left|\Omega_{i}\right|<<-1 \longrightarrow \alpha_{i j} \approx \delta_{i j}
$$


where $\Omega_{i}(i=1,2,3)$ represent the eigenvalues of $\Omega$. This may also apply $(0)$ materials displaying inherent anisotropy. As the failure criterion is approached the plastic strain associated with the rebuilding of the microstructure may obliterate the effect of inherent anisotropy, in which case $\alpha_{i j} \approx \delta_{i j}$.

The above conclusions are widely supported by experimental evidence. Over the last three decades a large number of tests were performed on isotropically and $\mathrm{K}_{\mathrm{o}}$-consolidated clay samples prepared from a slurry (e.g. I Ienkel and Sowal, 1963; Skempton and Sowa, 1963; Ladd, 1965; Lee and Morrison, 1970; Kirkpatric and Rennie, 1972). The results indicated that there was no influence of deformation history on the failure criterion in terms of effective stress. At the same lime, the tests on samples of undisturbed London clay (Barden, 1972), artificially prepared kaolin with oriented particles (Morgenstern and Tachlenko, 1967) or vertically and horizontally cut samıles of overconsolidated kaolin (Duncan and Seed, 1966) showed no influence of inherent anisotropy on the functional form of the fitilure criterion. It appears that in materials with small grain size (clay, silt, etc.) the fal)ric remains essentially isotropic, i.e. $\alpha_{i j} \approx \delta_{i j}$. On the other hand, the fabric anisotropy manifest itself strongly in media with relatively large grain size. There is some evidence that the inherent anisotropy affects both the failure criterion and the elastic response in materials like gravel with flat grains (Havlicek and Myslivec, 1965), sand with elongated grains (Oda, 1972), etc. 


\subsection{Description of anisotropy of plastic flow in materials with small grain size}

Let us concentrate now on the influence of material anisotropy on the irreversible deformation process (under $\alpha_{i j} \approx \delta_{i j}$ ). In such media, the bias in the spatial distribution of porosity, as defined by $\underline{\Omega}$, will inevitably affect the plastic flow. The idea of incorporating the tensor $\underline{\Omega}$ into constitutive framework is not unique. One way of formulating the problem is to assume that $\underline{\Omega}$ affects directly the functional form of the yield criterion. Then,

$$
\begin{aligned}
& \dot{\epsilon}^{p}{ }_{i j}=i \quad \frac{\partial f}{\partial \sigma_{i j}} \\
& f=f\left(\sigma_{i j}, \Omega_{i j}, æ\right)=0
\end{aligned}
$$

i.e., $f=0$ depends on the deformation history (recorded by $æ$ ) and ten (in general) functionally independent invariants of both tensors $\underline{\sigma}$ and $\underline{\Omega}$. It is interesting to note that, since the components of $\underline{\Omega}$ are derived from the total deviatroic strain $\underline{\mathbf{e}}$, the yield surface $f=0$ will undergo progressive evolution not only during an active loading process, but also for histories satisfying $\mathrm{f}<0$. Consequently, such a formulation will account for the influence of the rotation of the axes of principal stress, which invariably takes place in many practical geotechnical problems. 
An alternative approach, pursued in the subsequent development, may be based on resolving the plastic strain rate into components governing a pseudoisotropic response and divergence from it. It can be assumed, for this purpose, that as long as the material remains isotropic the deformation process can be described by a plasticity formulation incorporating the isotropic hardening rule, and that the deviations from isotropy are accounted for through

$$
\begin{aligned}
& \dot{\epsilon}^{p}{ }_{i j}=\dot{\lambda} \frac{\partial f}{\partial \sigma_{i j}}+\dot{\mu} G_{i j} \\
& f=f\left(\sigma_{i j}, æ\right)=0
\end{aligned}
$$

In equation(3.24) the first term in the flow rule represents the plastic strain rates generated in a fictitious isotropic medium, whereas $\mathbf{G}$ is a symmetric second order tensor whose components are function of $\underline{\Omega}$ and the deformation history $æ$

$$
G_{i j}=G_{i j}\left(\Omega_{k l}, æ\right)
$$

One of the simplest formulations of all will be obtained by taking

$$
G_{i j}=h \Omega_{i j}
$$

and 


$$
\dot{\mu}=\dot{\lambda}
$$

where $h$ is a scalar valued function of deformation history.

Substituting equation (3.27) and (3.28) into equation (3.24) yields

$$
\dot{\epsilon}_{i j}^{p}=\dot{\lambda}\left(\frac{\partial f}{\partial \sigma_{i j}}+h \Omega_{i j}\right)
$$

The above flow rule is analogous to a non-associated law and has, to some extent, similar numerical consequences to those of implementing a kinematic hardening. Namely, the deviations from isotropy result in a progressive deviation of the direction of the plastic flow from that specified by the gradient tensor $\partial f / \partial \sigma_{i j}$.

In order to derive the constitutive relation assume that the parameter $æ$ is identified with $\epsilon_{i i}^{p}$, i.e. plastic volumetric strain generated in a fictitious isotropic medium. 'Thus,

$$
\begin{aligned}
& i=f\left(\sigma_{i j}, \epsilon_{i i}^{p}\right)=0 \\
& \dot{\epsilon}_{i i}^{p}=\dot{\bar{\epsilon}}_{i i}^{p}=i \frac{\partial f}{\partial \sigma_{i i}}
\end{aligned}
$$

It should be noted that the relations (3.30) and (3.31) correspond to the well established Critical State concept. Writing the consistency condition 


$$
\dot{\mathbf{f}}=\frac{\partial \mathrm{f}}{\partial \sigma_{i j}} \dot{\sigma}_{i j}+\dot{\lambda} \frac{\partial \mathrm{f}}{\partial \bar{\epsilon}_{i \mathbf{i}}} \frac{\partial \mathrm{f}}{\partial \sigma_{k k}}=0
$$

and substituting the elasticity relationship

$$
\dot{\sigma}_{i j}=D_{i j k l}\left(\dot{\epsilon}_{k l}-\dot{\epsilon}_{k l}^{p}\right)
$$

yields

$$
\dot{\lambda}=\frac{1}{H} \frac{\partial f}{\partial \sigma_{i j}} D_{i j k l} \dot{\epsilon}_{k l}
$$

where

$$
H=\frac{\partial f}{\partial \sigma_{i j}} D_{i j k l}\left(\frac{\partial f}{\partial \sigma_{k l}}+h \Omega_{k l}\right)-\frac{\partial f}{\partial \bar{\epsilon}_{i i}^{p}} \frac{\partial f}{\partial \sigma_{k k}}
$$

Substitution of equation (3.34) back into equation (3.33) leads, after some algebraic manipulations, to the constitutive relation

$$
\dot{\sigma}_{i j}=\bar{D}_{i j k l} \dot{\epsilon}_{k l}
$$

where

$$
\bar{D}_{i j k l}=D_{i j k l}-\frac{1}{H} D_{i j p q}\left(\frac{\partial f}{\partial \sigma_{p q}}+h \Omega_{p q}\right) \frac{\partial f}{\partial \sigma_{r s}} D_{r s k l}
$$

The constitutive matrix defined by equation (3.37) has a similar form to that obtained using a non-associated flow rule. The deviation from normality, described 
in terms of $\underline{\Omega}$, allows for a simple and quite effective description of the effects of both inherent and induced anisotropy, as discussed in subsequent chapter. At the same time, the analysis is diversified by providing, through the function $\mathrm{n}(\mathbf{v})$, an insight into a deformation pattern on a microscale. The mathematical formulation incorporates one additional material function $h$, which can be identified from conventional "triaxial" tests. Details concerning specification of the yield function (3.32) are given in chapter 4.

Before leaving this section, an alternative approach can be briefly outlined by admitting a coupling between both terms in equation (3.24), instead of deviation from normality. Such a flow rule will be analogous to that derived in multi-yield loci theories, implying that the direction of plastic strain rate will be sensitive to the direction of stress rate.

Assume that the components of $\underline{\mathbf{G}}$, equation (3.26), are determined from a potential function

$$
\begin{aligned}
& \mathrm{g}=\mathrm{g}(\underline{\bar{\Omega}})=\text { const. } \\
& \bar{\Omega}_{\mathrm{ij}}=\mathrm{n}_{0}\left(\delta_{i j}+\Omega_{\mathrm{ij}}\right)
\end{aligned}
$$

where $\underline{\bar{\Omega}}$ is defined in such a manner that equation (2.18) becomes 


$$
\mathrm{n}\left(\mathrm{v}_{\mathrm{i}}\right)=\overline{\boldsymbol{\Omega}}_{\mathrm{ij}} \mathrm{v}_{\mathrm{i}} \mathrm{v}_{\mathrm{j}}
$$

Let the tensor $\underline{\mathbf{G}}$ be defined through the potential $\mathrm{g}(\underline{\underline{\underline{\Omega}}})$, i.e.,

$$
G_{i j}=h C_{i j k l} \frac{\partial g(\underline{\underline{\Omega}})}{\partial \bar{\Omega}_{k l}}
$$

where $C_{i j k !}$ represents the elastic compliance tensor and $h$ is again a scalar valued function of deformation history. Introduce a family of loading surfaces, i.e.,

$$
\mathrm{f}_{1}=\mathrm{f}_{1}(\underline{\underline{\Omega}})=\text { const. }
$$

defining the 'neutral' states for structural rearrangement as,

$$
\dot{\mu}=\frac{\partial \mathrm{f}_{\mathrm{q}}(\underline{\underline{\Omega}})}{\partial \overline{\mathrm{\Omega}}_{\mathrm{ij}}} \dot{\bar{\Omega}}_{\mathrm{ij}}
$$

Then, according to equation (3.24), for $\dot{\mu}>0$ there is

$$
\dot{\epsilon}_{i j}^{p}=i \frac{\partial f}{\partial \sigma_{i j}}+h C_{i j k l}\left(\frac{\partial g(\underline{\underline{\Omega}})}{\partial \bar{\Omega}_{k l}} \frac{\partial f_{q}(\underline{\underline{\Omega}})}{\partial \bar{\Omega}_{p q}}\right) \dot{\bar{\Omega}}_{p q}
$$


It should be noted that in the above flow rule, the plastic strain rates $\dot{\epsilon}_{i j}^{\prime}=\dot{\mu} G_{i j}$, equation (3.24), can be interpreted as being derived from residual stress rates $\dot{\sigma}^{R}{ }_{i j}$,

$$
\begin{aligned}
& \dot{\epsilon}_{i j}^{\prime}=C_{i j k l} \dot{\sigma}^{R k l} \\
& \dot{\sigma}_{k l}^{R}=h\left(\frac{\partial f_{1}(\underline{\underline{\Omega}})}{\partial \bar{\Omega}_{p q}} \dot{\bar{\Omega}}_{p q}\right) \frac{\partial g(\underline{\underline{\Omega}})}{\partial \bar{\Omega}_{k l}}
\end{aligned}
$$

Such an approach is analogous to strain-space plasticity formulation.

In order to derive the constitutive relation, let us express the evolution law for the components of $\underline{\bar{\Omega}}$ in the form

$$
\bar{\Omega}_{i j}=B_{i j k l} \dot{\epsilon}_{k l}
$$

where $B_{i j k l}$ is defined as

$$
B_{i j k l}=\left(1-n_{0}\right)\left(\delta_{i j}+\Omega_{i j}\right) \delta_{k l}+n_{0} B A_{i j k l}
$$

and

$$
B=g_{4}+g_{5} I_{2 n} \quad ; \quad A_{i j k i}=\delta_{k i} \delta_{i j}-(1 / 3) \delta_{i j} \delta_{k m} \delta_{t m}
$$


Substituting equation(3.45) into consistency condition (3.32) yields

$$
\begin{aligned}
\dot{\mathbf{f}}= & \frac{\partial \mathrm{f}}{\partial \sigma_{i j}} D_{i j k l}\left\{\dot{\epsilon}_{k l}-\dot{\lambda} \frac{\partial f}{\partial \sigma_{k l}}-\left(\frac{\partial \mathrm{f}_{1}(\underline{\bar{\Omega}})}{\partial \bar{\Omega}_{r s}} \dot{\bar{\Omega}}_{r s}\right) G_{k l}\right\} \\
& +\dot{i} \frac{\partial \mathrm{f}}{\partial \bar{\epsilon}_{i j}^{\mathrm{p}}} \frac{\partial \mathrm{f}}{\partial \sigma_{k k}}=0
\end{aligned}
$$

Thus, after some transformations, the plastic multiplier $\lambda$ is given by the following relation

$$
\dot{\lambda}=\frac{1}{H} \frac{\partial f}{\partial \sigma_{i j}}\left(D_{i j k l}-S_{i j k l}\right) \dot{\epsilon}_{k l}
$$

where,

$$
H=\frac{\partial f}{\partial \sigma_{i j}} D_{i j k l} \frac{\partial f}{\partial \sigma_{k l}}-\frac{\partial f}{\partial \bar{\epsilon}_{i i}^{p}} \frac{\partial f}{\partial \sigma_{k k}}
$$

and

$$
S_{i j k l}=h \frac{\partial g(\underline{\underline{\Omega}})}{\partial \bar{\Omega}_{i j}} \frac{\partial f_{q}(\bar{\Omega})}{\partial \bar{\Omega}_{p q}} B_{p q k l}
$$

Finally, following the same procedure to that used for deriving equation (3.37) one obtains 


$$
\vec{D}_{i j k l}=\left(\delta_{i j} \delta_{p q}-\frac{1}{H} D_{i j r s} \frac{\partial f}{\partial \sigma_{r s}} \frac{\partial f}{\partial \sigma_{p q}}\right)\left(D_{p q k l}-S_{p q k l}\right)
$$

It should be noted that the first term in equation (3.52) multiplied by $D_{\text {pqkt }}$ represents a conventional elastoplastic constitutive matrix and that the anisotropy effects enter through the components of $S_{\text {pqkt }}$.

The proposed constitutive relations, equations (3.37) and (3.52) are capable of describing the influence of inherent and induced anisotropy on the deformation process. In the subsequent chapter the performance of simplest description, i.e. defined by equation (3.37), is discussed and verified against the existing experimental data. 


\section{CHAPIER 4}

\section{NUMERICAL SIMULATIONS OF ANISOTROPY EIFFECIS IN NORMAII.Y AND LIGHTLY OVERCONSOLIDATED CI AYS}

\section{1 $\quad \underline{\text { Introduction }}$}

The framework described in the previous chapter is now illustrated by some numerical examples pertaining to normally consolidated and lightly overconsolidated clays. The simulations are based on the flow rule represented by equation (3.29) and the evolution law given by equation (2.24). In the subsequent section, 4.2 , the typical invariant measures of the effective stress state are bricfly reviewed, followed by the mathematical details concerning the specification of the functional form of the yield surface, $f=0$ (section 4.3 ). Section 4.4 refers to the applied numerical scheme for the integration of the elasto-plastic constitutive law. The developed numerical code allows to integrate the stress-strain relation along prescribed stress or strain paths, and covers two extreme cases of soil behaviour, i.e. fully drained and undrained response. In section 4.6 the results of the numerical simulations are presented and examined against the experimental data reported by 
Skempton and Sowa (1963), and Calladine (1971). The soil is assumed to be initially isotropic. The sets of parameters describing the material are specified in section 4.5 .

\subsection{Preliminary Definitions: Invariant Measures of the Effective Stress State.}

In the developed constitutive framework the rate of irreversible deformation, equation (3.24), has been resolved into components governing the pseudo-isotropic response and the divergency part. The direction of the plastic flow in a fictitious isotropic medium has been identified with the direction of the gradient vector of an isotropic yield function, symbolically denoted by equation (3.30). This function depends on the effective stress tensor $\underline{\sigma}$ and a single internal variable $æ$, which accounts for the past loading history.

According to the theorem of the representation of tensor functions, an isotropic scalar-valued function of a symmetric second order tensor is uniquely defined as a function of its basic invariants. The selection of a particular set of invariants is not unique, in a sense that any invariant measure (which is a function of basic invariants) may be employed. In the geotechnical practice, most experiments are completed in the 'triaxial' stress configuration (triaxial apparatus) 
or are referred to this configuration. A set of stress invariants which is compatible with static values measured in a triaxial cell and, at the same time, remains convenient for graphical representation was introduced by Nayak and Zienkiewicz (1972). These invariants are used in the subsequent development.

The first invariant is the mean effective stress ${ }^{1}$ I, i.e.,

$$
3 I=-\sigma_{i j}=-\left(\sigma_{x}+\sigma_{y}+\sigma_{z}\right)
$$

(Note: the compressive stresses and strains are considered negative).

The second invariant $\sigma$ and the angular measure of the third invariant $\theta$, are derived from the stress deviator $\underline{\mathbf{s}}$, i.e.,

$$
\sigma=\left[\frac{y}{z} s_{i j} s_{i j}\right]^{y / z}=\left[b_{z}\left(s_{x x}{ }^{2}+s_{y y}{ }^{2}+s_{z z}^{2}\right)+\sigma_{x y}^{2}+\sigma_{y z}^{2}+\sigma_{z x}^{2}\right]^{y}
$$

and

$$
\theta=\frac{1}{3} \arcsin \left[-\frac{3 \sqrt{2}}{2} \frac{\mathrm{J}_{3}}{\sigma^{2}}\right]
$$

with the limits imposed on $\theta$ angle, $-\pi / 6 \leq \theta \leq \pi / 6$.

In the above definitions, $\mathrm{J}_{3}$ is the third invariant of $\mathbf{s}$, i.e.,

$$
\mathrm{J}_{3}=1 / 3 \mathrm{~s}_{\mathrm{ij}} \mathrm{s}_{\mathrm{jk}} \mathrm{s}_{\mathrm{ki}}=\mathrm{s}_{\mathrm{xx}} \mathrm{s}_{\mathrm{y} y} \mathrm{~s}_{z z}+2 \sigma_{\mathrm{xy}} \sigma_{\mathrm{yz}} \sigma_{z x}-\mathrm{s}_{\mathrm{xx}} \sigma_{y z}{ }^{2}-\mathrm{s}_{y y} \sigma_{x z}{ }^{2}-\mathrm{s}_{z z} \sigma_{x y}{ }^{2}
$$

${ }^{1}$ in the subsequent definitions tensor $\underline{g}$ indicates the effective stress tensor. 
In the principal stress space $\Theta$ represents an angle defined in the deviatoric plane $(\pi)$, i.e., a plane which is perpendicular to the hydrostatic axis $\left(\sigma_{1}=\sigma_{2}=\sigma_{3}\right)$. Hence, in the $\pi$-plane the first invariant $I$ remains constant and a yield function represents a locus of points whose distances from the hydrostatic axis have the magnitude of $\sqrt{2} \sigma$. In addition, the $\pi$-plane section of an isotropic yield surface forms six symmetric sectors and the limits imposed on $\theta$ represent the span of these sectors.

If a yield function is written in terms of the effective stress invariants (I, $\bar{\sigma}, \theta)$, i.e.,

$$
f=f(I, \bar{\sigma}, \Theta, x)=0
$$

then its gradient is defined by

$$
\frac{\partial f}{\partial \sigma_{i j}}=\frac{\partial f}{\partial I} \frac{\partial I}{\partial \sigma_{i j}}+\frac{\partial f}{\partial \bar{\sigma}} \frac{\partial \bar{\sigma}}{\partial \sigma_{i j}}+\frac{\partial f}{\partial \theta} \frac{\partial \theta}{\partial \sigma_{i j}} .
$$

The above expression can be also written in a vectorial form, more suitable for numerical applications, i.e.,

$$
\frac{\partial \mathrm{f}}{\partial \underline{\sigma}}=C_{1} \underline{b}_{1}+C_{2} \underline{b}_{2}+C_{3} \underline{b}_{3}
$$


where $C_{1}, C_{2}, C_{3}$ are scalar arguments and $\underline{b}_{1}, b_{2}, b_{3}$ are interpreted as vectors in a six-dimensional effective stress space. The specification of these measures, for the yield function introduced in section 4.3, is discussed in Appendix A.

Finally, the efiective principal stresses are related to the stress invariants by,

$$
\left\{\begin{array}{l}
\sigma_{1} \\
\sigma_{2} \\
\sigma_{3}
\end{array}\right\}=\frac{2 \bar{\sigma}}{\sqrt{3}}\left[\begin{array}{c}
\sin \left(\theta+\frac{2 \pi}{3}\right) \\
\sin \theta \\
\sin \left(\theta+\frac{4 \pi}{3}\right)
\end{array}\right]+\left\{\begin{array}{l}
\mathrm{I} \\
\mathrm{I} \\
\mathrm{I}
\end{array}\right\}
$$

where $\sigma_{1}>\sigma_{2}>\sigma_{3}$.

\subsection{Specification of the yield function.}

As pointed out by Drucker (1966), the selection of the specific functional form of the yield surface is to a large extent wide-open. The choice depends primarily on the convenience in the experimental verification and in the subsequent numerical implementation. The forms of the yield function advocated in literature are usually associated with the assumption about both the character of the plastic 
flow and the plastic hardening rule. The onset of the plastic flow is defined either, as a combination of yield points identified from experimental stress-strain plots, or on the base of reasoning about the character of the dissipation work equation (e.g.: Burland, 1965; Podgorski, 1985; Lade and Kim, 1988).

In the stress reference frame $I, \bar{\sigma}, \theta$, an isotropic yield surface can be completely defined by specifying its cross-sectional contour in the deviatoric plane ( $\pi$-plane) and its meridian section in the $I-\bar{\sigma}$ subspace. The shape of the contour in the deviatoric plane can be described through the function $g(\theta)$. This function is defined as the ratio of the yielding point distances, i.e.,

$$
g(\theta)=\frac{\bar{\sigma}(\theta)}{\bar{\sigma}_{c}}
$$

Here $\bar{\sigma}(\theta)$ is proportional to the current distance of the yield locus from the hydrostatic axis, whereas $\bar{\sigma}_{c}$ is a maximum distance measured in 'triaxial' compression $(\theta=\pi / 6)$; both distances are evaluated at a constant confining pressure I.

The influence of the shape of the deviatoric cross-section on the computational results pertaining to the bearing capacity problem, was examined by Zienkiewicz and Pande (1977). Recently, the issue of the convexity of various 
$\pi$-plane idealizations has been addressed by Jiang and Pietruszczak (1988). The latter work demonstrates that, in the context of an isotropic plastic solid, the convex shape of the deviatoric contour is a physical rather than a mathematical requirement. Indeed, in the case of an associated plastic flow, the criterion of the positive rate of the energy dissipation does not require convexity of the yield surface in the octahedral plane, i.e., the irreversibility condition does not depend on $\theta$. Thus, the convexity requirement seems to be more critical in the context of the limit state analysis, where it is indispensable in proving the limit theorems. On the other hand however, the concave shape of the deviatoric cross-section has not yet been detected by experimentalist. Therefore, Jiang and Pietruszczak (1988) propose a novel and less complicated idealization of the $g(\theta)$ function. This idealization, which provides a convex shape of the $\pi$-plane section, has been used in the subsequent development.

It can be assumed that the yield locus in deviatoric $\pi$-plane can be represented by the following function

$$
g(\theta)=\frac{K(\sqrt{1+a}-\sqrt{1-a})}{K \sqrt{1+a}-\sqrt{1-a}+(1-K) \sqrt{1-a} \sin 3 \theta}
$$

The function $\mathrm{g}(\theta)$ is defined in such a manner that $\mathrm{g}(\pi / 6)=1$ and $\mathrm{g}(-\pi / 6)=\mathrm{K}$. The parameter $\mathrm{K}$ denotes the ratio of deviatoric stress intensities in 'triaxial' 
extension and compression, as illustrated in Figure 4.1., and is related to the angle of internal friction $\Phi$ by the expression

$$
K=(3-\sin \Phi) /(3+\sin \Phi)
$$

The function $g(\theta)$, equation (4.9a), describes a noncircular convex cross-section in the deviatoric plane. The sensitivity of the function $g(\theta)$ to the confining pressure can be accounted for by assuming $K=K(I)$. For the value of $K$ close to $\frac{1}{2}$ the $\pi$ plame cross-section degenerates into a curvelinear triangle and resumes a nearly circular shape for the K's approaching 1, as illustrated in Figure 4.2. It should be noted that for $a=1$ the derivative $\partial \mathrm{g} / \partial \theta$ is singular, however this deficiency can be controlled by selecting a -1 .

In order to complete the description of the yield surface its meridional section must be specified. Assume that in the meridional plane $(\mathrm{I}-\bar{\sigma} / \mathrm{g}(\theta))$ the yield function is asymmetric and consists of two parts; i.e. the segment of a parabola, represented by

$$
f_{p}=\bar{\sigma}+\frac{\eta g(\theta)\left(I_{o}-I_{x}\right)}{I_{x}^{2}}\left[\left(I-I_{x}\right)^{2}-I_{x}^{2}\right]=0
$$


and an elliptical cap

$$
f_{e}=\bar{\sigma}^{2}+\eta^{2} g^{2}(\theta)\left[\left(I-I_{x}\right)^{2}-\left(I_{0}-I_{x}\right)^{2}\right]=0
$$

Such a concept is similar to that proposed by Sandler, DiMagio and Baladi (1970) and permits a considerable leeway in the modelling of the plastic flow of geological materials. The elliptical cap may expand or contract owing to the increase of decrease in the parameter $I_{0}$, i.e.,

$$
I_{0}=I_{0(0)} \exp \left(\frac{e_{0}^{p}-e^{p}}{\lambda-\kappa}\right)
$$

where, $\dot{\mathrm{e}}^{\mathrm{p}}=\left(1+\mathrm{e}_{0}\right) \dot{\boldsymbol{\epsilon}}_{\mathrm{i}}^{\mathrm{p}}$ and $\lambda, \kappa$ are material constants identified from hydrostatic compression test. The cap's curvature is controlled by the parameter $\eta$. The merging point of both surfaces travels along the critical state line

$$
\eta_{f}=g(\theta)[(6 \sin \Phi) /(3-\sin \Phi)]
$$

,i.e., the line defining the state of soil at which the progressing shearing is accompanied by no irreversible volume change (cf. Schofield and Worth; 1968). The requirement of the continuous coalescence of both segments results in the subsequent relation for the position of the merging point

$$
\mathrm{I}_{\mathrm{x}}=\frac{\eta \mathrm{I}_{0}}{\left(\eta+\eta_{\mathrm{f}}\right)}
$$


It should be noted that, equation (4.10) is relevant only to cohesionless soils. In order to incorporate cohesion effects the following transformation of the stress tensor $\underline{\sigma}$ can be introduced (cf. Lade and Kim, 1988),

$$
\sigma_{i j}=\sigma_{i j}+\delta_{i j} I_{*}
$$

This transformation is equivalent to the translation of the apex ( $\left.O^{\prime}\right)$ of the surface along the hydrostatic axis by the quantity $I_{*}$. The differentiation of the yield function written in terms of $\underline{\sigma}$, and the use of the transformation (4.14) results in the following consistency equation

$$
\dot{f}=\frac{\partial f}{\partial \sigma_{i j}} \dot{\sigma}_{i j}+\left(\frac{\partial f}{\partial I_{\star}} \frac{\partial I_{\star}}{\partial \dot{x}}+\frac{\partial f}{\partial I_{0}} \frac{\partial I_{0}}{\partial \dot{x}}\right) \dot{x}=0
$$

Here, the quantity $I_{*}$ is further assumed to depend on the deformation history experienced by soil, i.e.,

$$
I_{*}=I_{*}(x)
$$

From equation (4.15) one can observe that, the general form of the flow rule (3.26) remains valid, although the derivatives should now be defined in terms of translated stresses. Similarly, the expression for the hardening modulus $H_{p}$ ought to be modified consistently with the assumption (4.16). Appendix A provides the 
appropriate expressions for the derivatives, as weil as $H_{p}$, assuming that the parameter $æ$ is identified with $\epsilon_{i f}^{p}$, i.e. the plastic volumetric strain generated in a fictitious isotropic medium.

Figure 4.2 shows the meridional cross-section of the yield surface, equation (4.10). This surface is convex and smooth, with the exception of the point $O^{\prime}$ where the discontinuity in $\partial \mathrm{f} / \partial \mathrm{I}$ exists. Thus, the proposed form overcomes the singular nature of the Cam-Clay yield function (at $I=I_{0}$ ) and at the same time provides a similar accuracy for describing soil response during undrained deformation programs. In addition, for the plastic flow at confining pressures less then $\mathrm{I}_{\mathrm{x}}$ the yield function defined by equation (4.10) provides the decrease of the dilatancy angle as the critical state is approached.

4.3 Numerical procedure for the integration of constitutive relation,

The constitutive relations developed in Chapter 3 are nonlinear, i.e. both stiffness and compliance tensors depend on the current state of stress, the fabric tensor as well as the deformation history experienced by the material. In general, the solution to a boundary value problem, employing a non-linear stress-strain law, is of an approximate nature; loads are applied in finite increments and usually the 
set of the governing differential equations is satisfied within a certain limit of error. Several techniques for the solution of non-linear problems of plastic solids have been developed in the past two decades (Snyder and Bathe, 1981), and are commonly classified as: tangential stiffness, initial strain/stress method (Nayak and Zienkiewicz, 1972) or the pseudo visco-plastic approach (Zienkiewicz and Cormou, 1974). The contributions aimed at improving the efficiency of these methods are still of interest to researchers (DeBorst and Vermeer, 1984; Runesson, 1987; Stolle and Higgins, 1989).

In order to carry out the numerical simulations, a simple code has been developed, allowing to integrate the proposed elasto-plastic constitutive law, at a material point, along a specified stress or strain path. The code has been based on the most straightforward numerical scheme for solving a set of nonlinear equations, known as the Euler Method. By expanding an unknown function in a truncated Taylor series, the values of the function after each load increment are foreseen using the values at the end of the previous loading step. Both the accuracy and the stability of the achieved solution are affected by the size of the load step, which must be kept appropriately small. The sequence of thie computational steps, during a representative load increment, is provided on the flow chart in Figure 4.4.

The presented procedure enables one to analyze the two extreme cases of soil behaviour: the fully drained (long lasting load) and the undrained (rapid load) case. In the fully drained process the seepage of the pore fluid is unconstrained, 
thus the excess of pore pressure is dissipated and the applied load is fully transmitted to the soil skeleton. Numerically, this type of the load program can easily be handled by both the stress and the strain controlled version of the developed code. In the undrained process the permeability of soil is not sufficient, as compared to the velocity of the loading, to allow the dissipation of the excess of pore pressure, in which case the soil skeleton and the pore fluid deform together. Although, the numerical integration of this process along the specified strain path is unambiguous, the solution for the prescribed total stress history confronts some difficulties, as the increment of the pore pressure must be estimated at the beginning of each load increment. This difficulty however can be overcome by the following reasoning.

Owing to the Mass Conservation Equation, the rate of the pore pressure build-up is related to the volumetric part of the strain rate by the equation,

$$
\dot{\mathrm{u}}=\mathrm{K} \delta_{\mathrm{ij}} \dot{\epsilon}_{\mathbf{i j}}
$$

where, $\mathrm{K}$ denotes the bulk modulus of soil skeleton. By equating the sum of the volumetric strain in the fluid and the solid phase to the total change in the volumetric strain, the bulk modulus $\mathrm{K}$ can be expressed as a function of: soil porosity $n_{o}$, the bulk moduli of soil particles $K_{s}$ and pore fluid $K_{f}$,i.e.,

$$
\frac{1}{K}=\frac{n_{o}}{K_{f}}+\frac{1-n_{0}}{K_{s}}
$$


Further, assuming that, the deformation of the soil skeleton is described by the 'rate-independent' plasticity formulation and soil grains are incompressible, the rate of pore pressure generation can be related to the rate of total stresses by the following equation (cf. Pande and Pietruszczak, 1989),

$$
\dot{\mathrm{u}}=\frac{\delta_{k l} \mathrm{C}^{\mathrm{ep}} \mathrm{klij}}{\delta_{r s} \mathrm{C}_{r s p q}^{\mathrm{ep}}{ }_{\mathrm{rqq}}+\left(\mathrm{n} / \mathrm{K}_{f}\right)} \dot{\sigma}^{\top}{ }_{\mathrm{ij}}
$$

Here, $\underline{C}^{\text {ep }}$ is an elasto-plastic compliance tensor whose components are function of effective stresses. For example, in a case of the flow rule described by equation (3.31) the constitutive tensor relating the strain rate to the rate of effective stresses has the form,

$$
\mathrm{C}^{\mathrm{ep}}{ }_{i j k l}=\mathrm{C}^{\mathrm{e}}{ }_{i j k l}+\frac{1}{\mathrm{H}_{p}}\left[\frac{\partial \mathrm{f}}{\partial \sigma_{i j}}\left(\frac{\partial \mathrm{f}}{\partial \sigma_{k l}}\right)+\mathrm{h}\left(\frac{\partial \mathrm{f}}{\partial \sigma_{k l}}\right) \Omega_{i j}\right]
$$

Thus, using equation (4.19) together with equation (4.20) an estimate of the pore pressure build-up due to the total stress increase can be obtained at the beginning of each load step. It should be noted that in most cases the pore fluid can be assumed as incompressible, $K_{f} \rightarrow \infty$, which results in further simplification of equation (4.19). 
In the explicit integration scheme the effective stresses have to be corrected after each load increment in order to satisfy the consistency condition. Since there is no inherent information in the plasticity formulation suggesting how the correction process should be performed, various strategies are possible. In the developed code, the approach analogous to that suggested by Nayak and Zienkiewicz (1972) has been employed, as briefly described below.

Assume that the direction of the stress correction is normal to the yield surface, i.e. the stress state satisfying the instantaneous yield locus can be written symbolically as,

$$
\underline{\sigma}_{(n+1)}=\underline{\sigma}_{(n+1)}^{\circ}+\alpha\left(\frac{\partial \mathrm{f}}{\partial \underline{\sigma}}\right)
$$

Here, $\underline{\sigma}_{(n+1)}^{\circ}=\underline{\sigma}_{n}+\Delta \underline{\sigma}_{n}$ and $\alpha$ denotes the unknown yet magnitude of the correction. Now, using the truncated Taylor expansion, the successive estimates of $\alpha$ can be calculated from the following formula

$$
\alpha_{i}=\alpha_{i-1}-\frac{f\left(\alpha_{i-1}\right)}{\partial f\left(\alpha_{i-1}\right) / \partial \alpha}
$$

where, the derivative $\partial f\left(\alpha_{i-1}\right) / \partial \alpha$ is equal to 


$$
\frac{\partial f}{\partial \alpha}=\frac{\partial f\left(\alpha_{i-1}\right)}{\partial \underline{\sigma}} \frac{\partial \underline{\underline{\sigma}}}{\partial \alpha}
$$

In order to avoid an excessive drift of stress state from the instantaneous yield surface, a number of successive iterations has to be completed until the prescribed tolerance is reached, i.e.,

$$
\left|f\left(\alpha_{i}\right)-f\left(\alpha_{i-1}\right)\right|<\text { tolerance }
$$

\subsection{Identification of material parameters.}

Implementation of equation (3.39) in a numerical code requires specification of material functions involved. Thus, in addition to parameters associated with isotropic hardening, both the evolution law $\Omega_{i j}=\Omega_{i j}\left(\Omega_{k l}, e_{k l}, n_{o}\right)$ and the material function $h$, equation (4.21), must be identified. The evolution law, equation (2.24), should be specified a priori based on an appropriate experimental program. The procedure suggested for this purpose has been briefly outlined in Chapter 2. Once the equation (2.24) has been quantified, the function $\mathrm{h}$ can be selected from conventional 'triaxial' tests. In what follows, constitutive relation (3.39) has been applied to simulate the response of saturated Weald clay and 
(3.39) has been applied to simulate the response of saturated Weald clay and Spestone kaolin.

At this stage, the existing experimental evidence is insufficient to identify, in a unique manner, the parameters entering the evolution law (2.24). Therefore, for both clays the values of these parameters have been selected on purely intuitive basis. This, somewhat arbitrary choice, may lead to errors in the spatial distribution of the void ratio. On the other hand however, the overall performance of the model may not be significantly affected, as both parameters A and B are chosen prior to the identification of the function $h$, equation (3.29). Finally, the function $h$ has been assumed in the form,

$$
h=h_{0}\left[1-\left(\frac{\bar{\sigma}}{g(\theta) I}\right)^{\gamma}\right]
$$

where $\mathrm{I}, \bar{\sigma}$ and $\theta$ represent the stress invariants defined in section 4.2. The constants $h_{0}$ and $\gamma$ may be identified from conventional 'triaxial' tests (trial and error procedure). For example, $\mathrm{K}_{\mathrm{o}}$-consolidation and drained uniaxial compression may be suitable for this purpose. The estimates of ho and for the numerical simulations discussed in section 4.5 are given in section 4.4 .1 and 4.4 .2 , respectively. 


\subsubsection{Weald clay.}

The experimental data pertaining to this clay are provided by Skempton and Sowa (1963). The material parameters corresponding to the Critical State concept (see section 4.2) have been chosen as: $\kappa=0.03, \nu=0.27, \lambda=0.091$, $\Phi=26^{\circ}, \mathrm{e}_{0}=0.95$ (at initial hydrostatic pressure of $50 \mathrm{kPa}$ ). Moreover, the void ratios corresponding to liquid and shrinkage limits were estimated as $e_{\mathrm{L}}=1.25$, $e_{s}=0.10$, respectively. The values of the parameters entering the evolution law have been selected as, $\mathrm{A}=3.0$ and $\mathrm{B}=1.0$. Subsequently, the constants $h_{0}=-6.0$ and $\gamma=0.35$ have been estimated by a trial and error procedure from the $\mathrm{K}_{0}$-consolidation and the drained uniaxial compression test.

\subsubsection{Spestone kaolin.}

The physical and mechanical properties of this clay has been described by Roscoe and Poorooshasb (1963), and subsequently cited by Calladine (1971). The set of the parameters corresponding to Critical State concept have been selected as: $\kappa=0.06, \nu=0.30, \lambda=0.30, \Phi=23^{\circ}, \mathrm{e}_{0}=1.40$ (at initial hydrostatic pressure of $100 \mathrm{kPa}$ ). The void ratios defining the porosity index, equation (2.25), have been recalculated as $\mathrm{e}_{\mathrm{L}}=1.83, \mathrm{e}_{\mathrm{s}}=0.21$, respectively. The values of the 
parameters defining the evolution law (2.24) have been chosen as, $\mathrm{A}=3.0$ and $B=1.0$, whereas the constants $h_{0}=6.0$ and $\gamma=0.35$ have been estimated by the procedure similar to that described in section 4.4.1.

4.5 Numerical simulations: anisotropy induced by the $\mathrm{K}_{0}$-consolidation process.

Restricting the attention to an inherently isotropic material, it can be assumed that the deformation process has a negligible effect on the material fabric, i.c. $\alpha_{i j}=\delta_{i j}$ in equation (3.1), and that the influence of induced anisotropy is described by constitutive relation (3.39). The results of numerical simulations are presented in Figures 4.5 to 4.9 . The study concentrates on 'triaxial' stress programs following $\mathrm{K}_{0}$-consolidation. The behaviour during $\mathrm{K}_{0}$-consolidation is of a special interest, since most natural soils are originally deposited under these conditions.

ligurc 4.5 shows the effective stress paths describing the one-dimensional consolidation followed by the undrained compression. The numerical simulations correspond to the original Critical State model and were completed for two different isotropic yicld functions, defined by equations (B.1) and (B.2) in Appendix B. It is evident that the values of $\mathrm{K}_{0}$ are significantly overpredicted and the associated errors influence subsequent material behaviour under undrained constraint. 
Figure 4.6 presents the simulations based on the proposed constitutive law (3.39), employing the yield function (4.10). Here, the parameters quantifying the material function h, equation (4.25), have been selected to provide an accurate representation of the stress path, as shown in Figure 4.6a. Figure 4.6b shows a progressive evolution of the spatial distribution of void ratio during $\mathrm{K}_{0}$-consolidation. As mentioned before, the results are quite intuitive as no experimental information is available. As reported by Skempton and Sowa (1963), the average void ratio at the end of consolidation process was approx. 0.57 , which agrees quite reasonably with the distribution in Figure 4.6b.

The $\mathrm{K}_{0}$ - consolidation process results in an induced anisotropy which is displayed through a significant bias in the directional distribution of void ratio. This anisotropy, which is of the nature of a cross-anisotropy, will influence subsequent material behaviour, as illustrated in figures below. Figure 4.7 shows a hypothetical response during hydrostatic loading which follows $\mathrm{K}_{0}$-consolidation and undrained unloading (sampling procedure). The strain rate field within the sample is no longer hydrostatic as the generation of deviatoric strains takes place. Strain rates in the radial direction are higher than those in the axial direction, which seems qualitatively correct (see Khera and Krizek, 1968).

Figure 4.8 shows a hypothetical undrained response of specimens trimmed at different orientations relative to the direction of the major consolidating stress. The undrained process follows $\mathrm{K}_{0}$-consolidation and unloading to a hydrostatic 
stress state. The simulations for the reloading process have been completed by employing the franework of bounding surface plasticity as described by Dafalias and Herman (1982) with the interpolation function, for the field of hardening moduli, identical to that used by Pietruszczak and Mroz (1982); $\mathrm{H}_{\mathrm{po}}=3.0 \times 10^{5} \mathrm{kPa}$ and $\Gamma=4.0$. The anisotropy in the material microstructure, as induced during $\mathrm{K}_{0}$-consolidation, results in noncoincidence of effective stress paths for vertical and horizontal specimens, Figure $4.8 \mathrm{~b}$. The response of the vertical specimen is stiffer, Figure $4.8 \mathrm{a}$, and its undrained strength is marginally higher than that of the horizontal one. The predicted trends are in qualitative agreement with the existing experimental data (e.g. Khera and Krizek, 1968; Mitchell, 1972).

Finally, Figure 4.9 shows the simulations of a stress probe experiment. The state of stress is brought to a common point following three different deformation histories, i.e., $\mathrm{K}_{0}$-consolidation, drained uniaxial compression and undrained compression. The stress probes, which are extensions of the previous histories, results in different orientations of the direction of the plastic flow. The results are consistent with those reported by Calladine (1971) and cannot be predicted by any isotropic-hardening theory. 


\subsection{Conclusions}

The numerical results, although still fragmentary, clearly demonstrate the ability of the described framework to model some typical effects of strain induced anisotropy in particulate media. The employed continuous measure of fabric, which is identified with the spatial distribution of porosity/void ratio, offers substantial improvements in the phenomenological description of soils. At the same time, the formulation remains physically descriptive, i.e., the material response is an implicit function of the specific manifestation of the microstructure. 
(a)

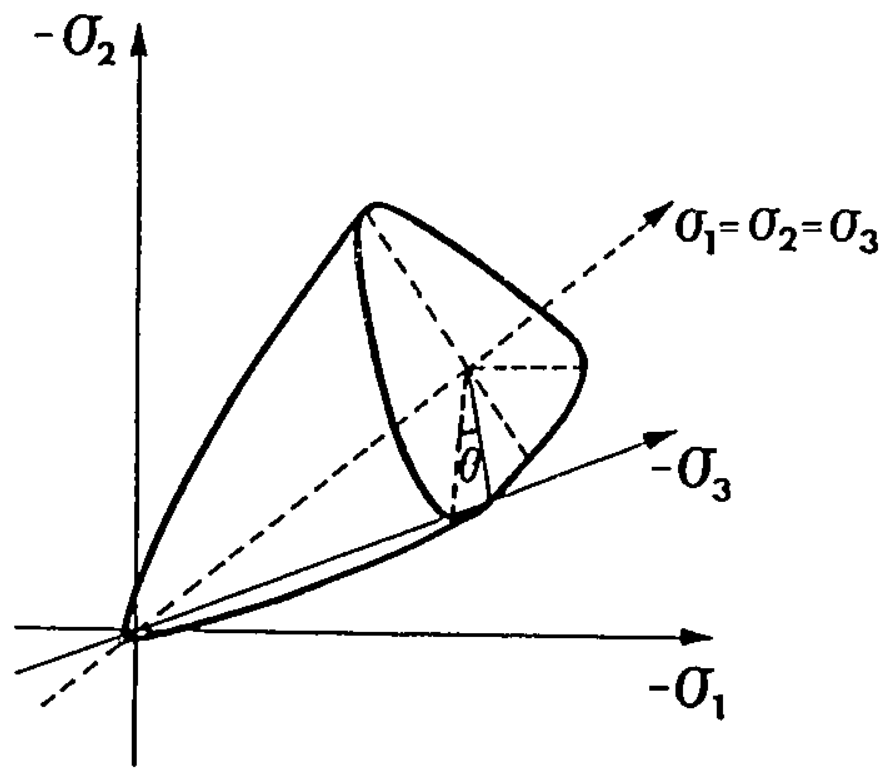

(b)

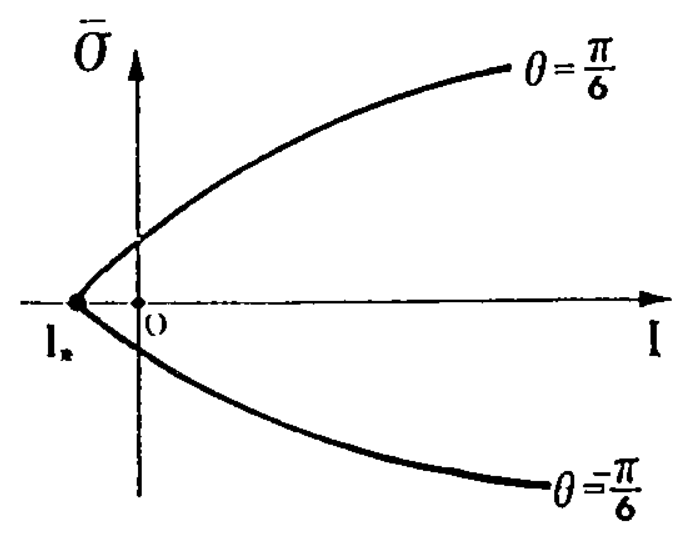

(c)

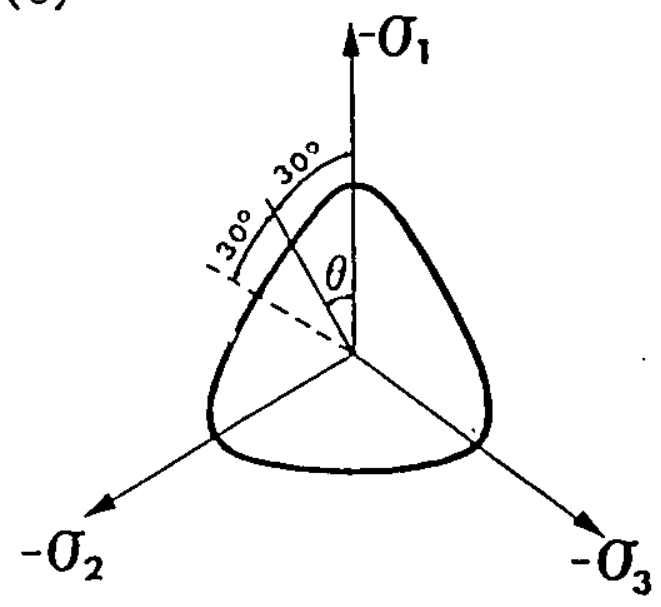

Figure 4.1 Typical yield surface for soils in the principal sress space; (b) meridional plane, (c) deviatoric plane. 


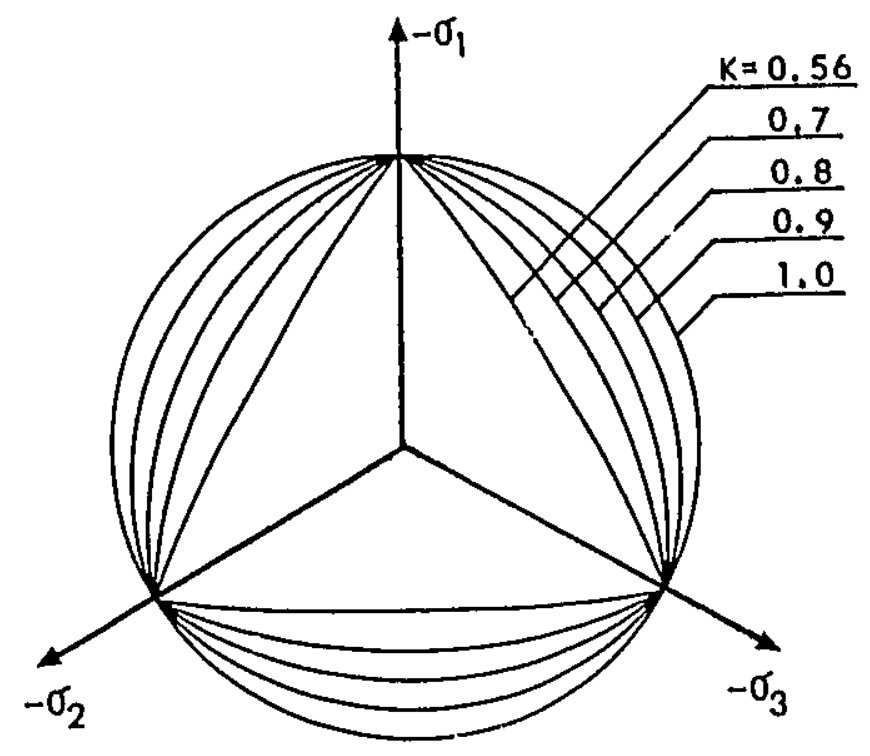

Figure 4.2 Deviatoric cross-section generated by equation (4.9) for different values of parameter $K$ (Jiang and Pietruszczak, 1988)

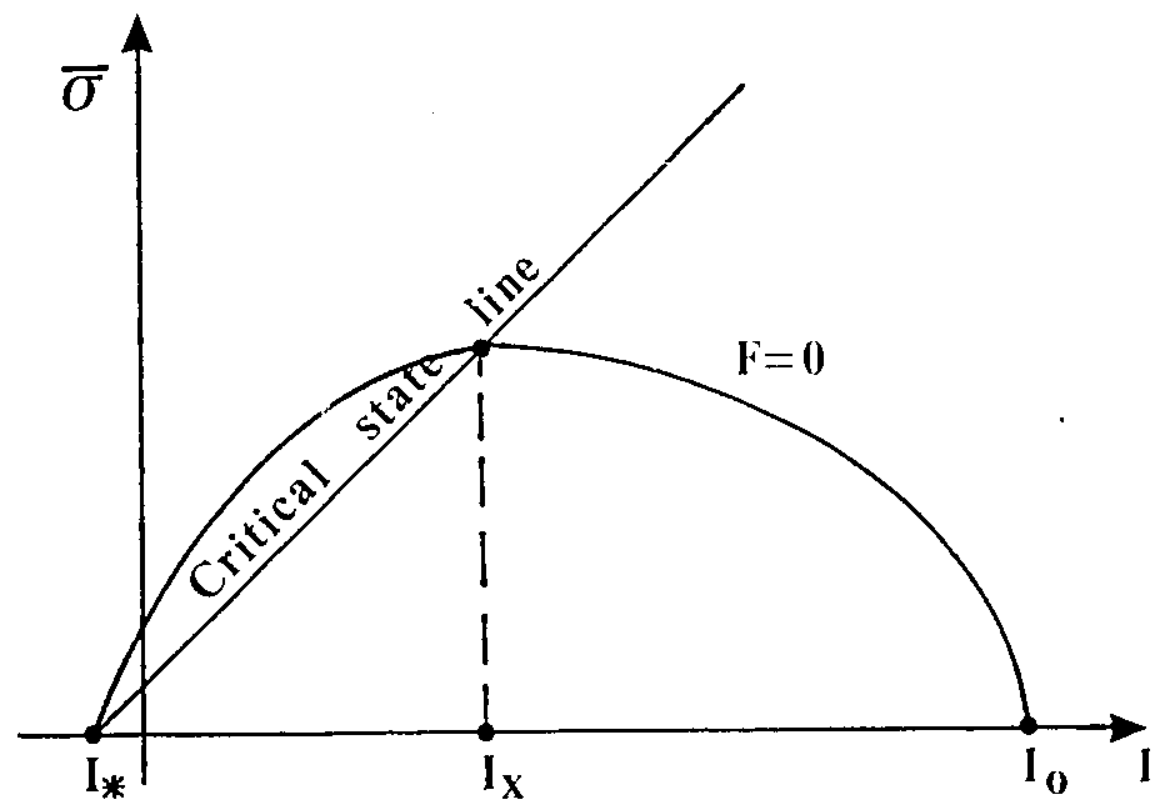

Figure 4.3 Meridional cross section of the yield function described by equation (4.10). 


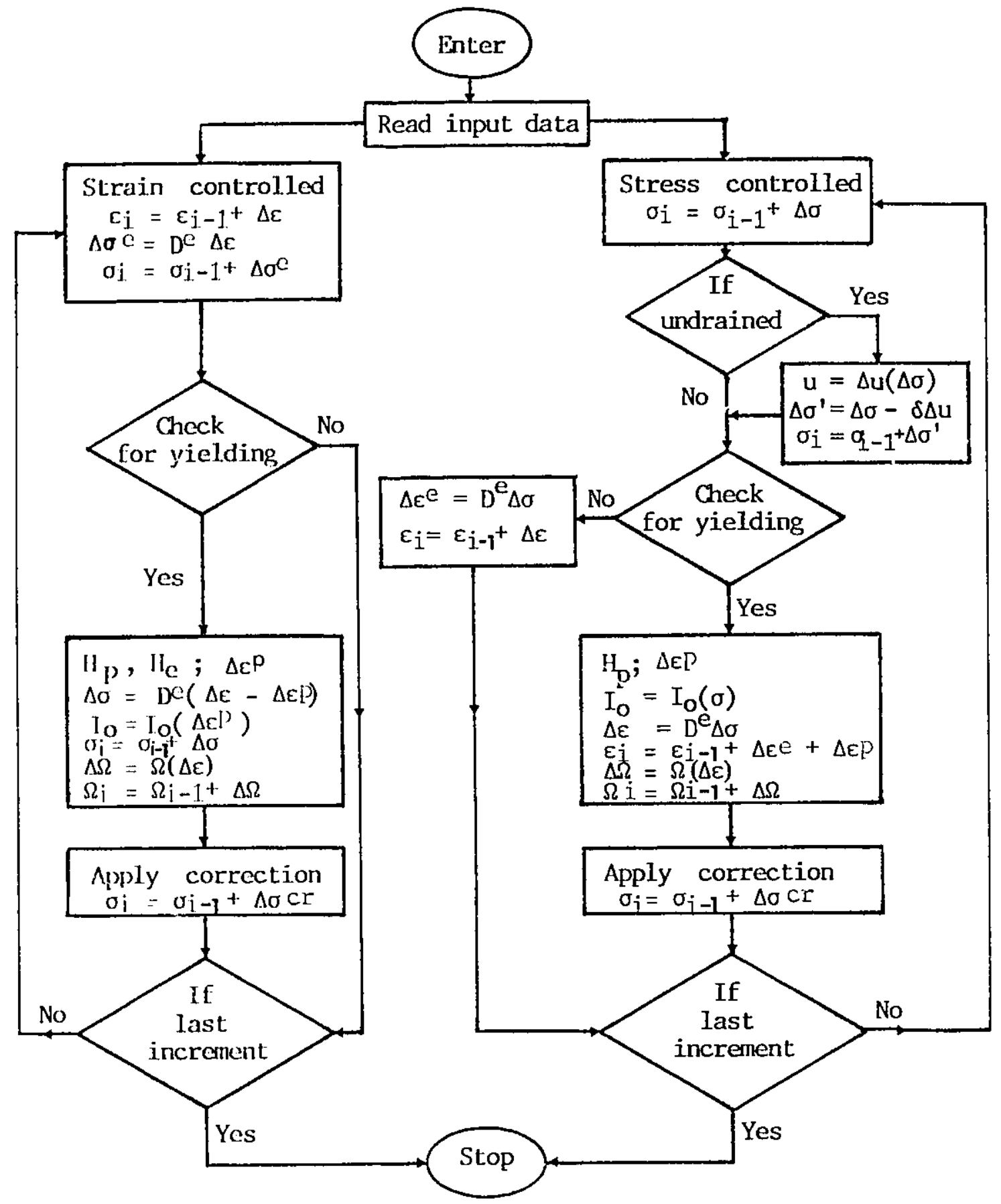

Figure 4.4 Flow chart of numerical code for integration of the proposed elasto-plastic constitutive law. 


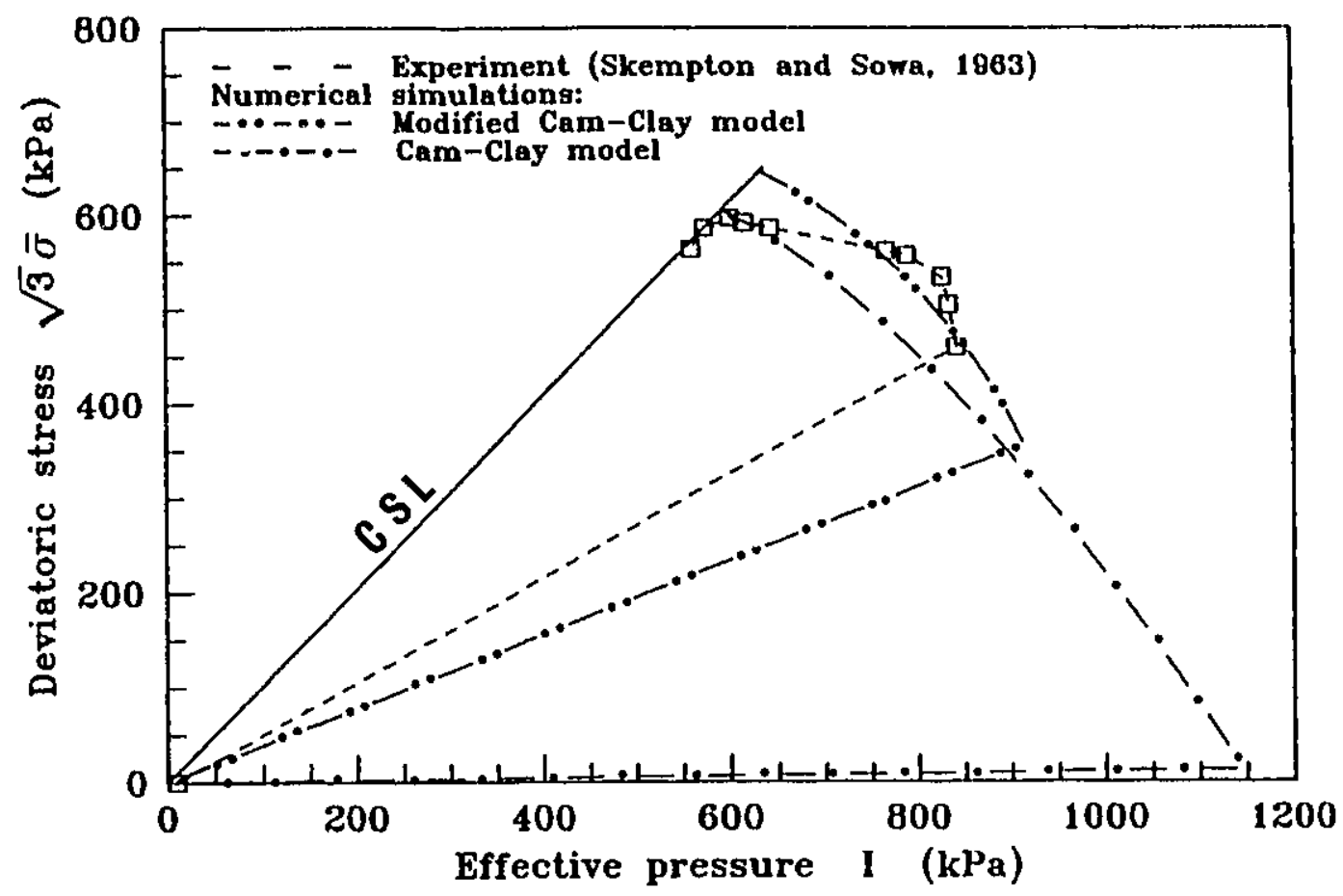

Figure 4.5 Effective stress paths for Ko-consolidation followed by undrained uniaxial compression. 


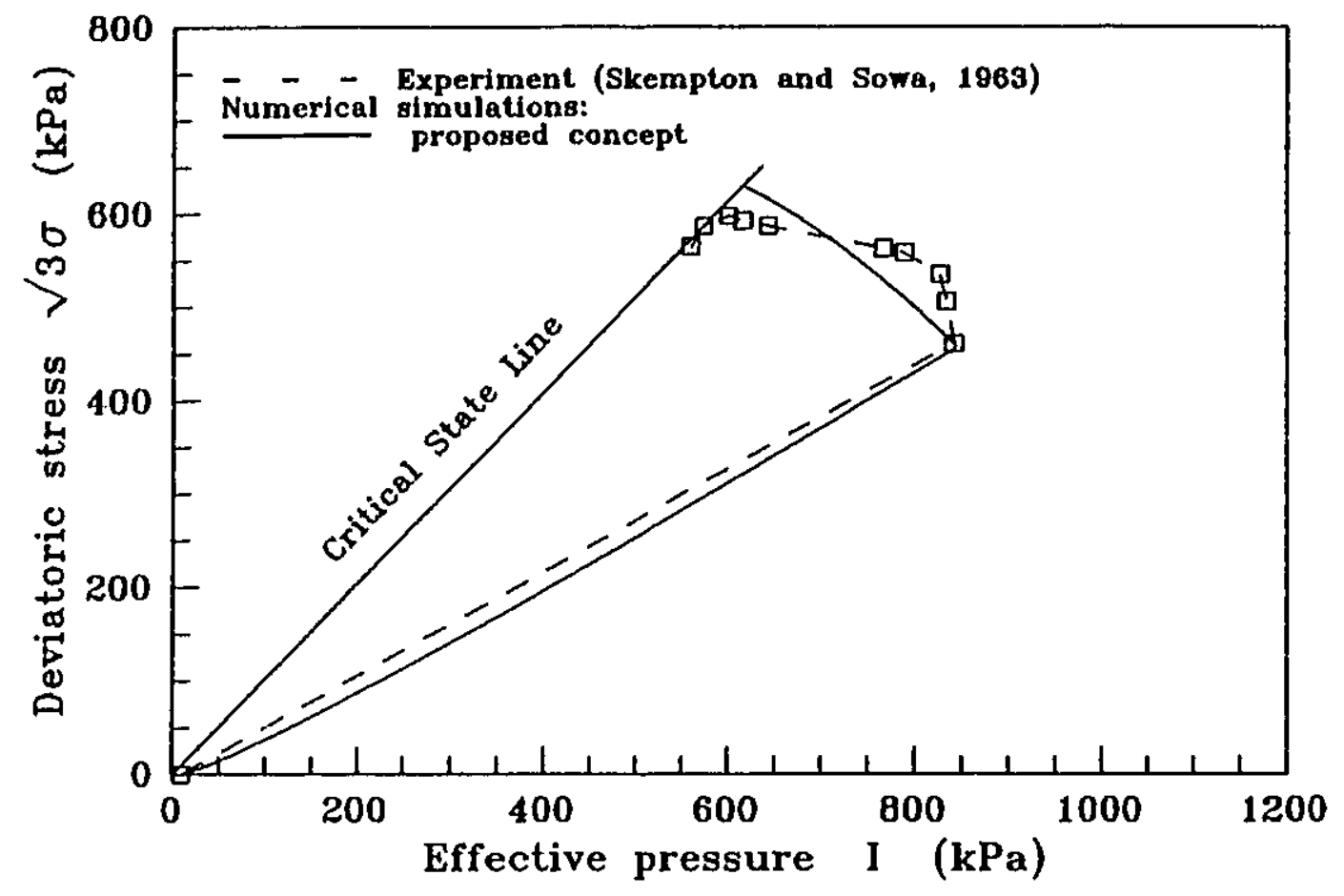

Figure 4.6a Effective stress paths for $K_{O}$-consolidation followed by undrained uniaxial compression. 


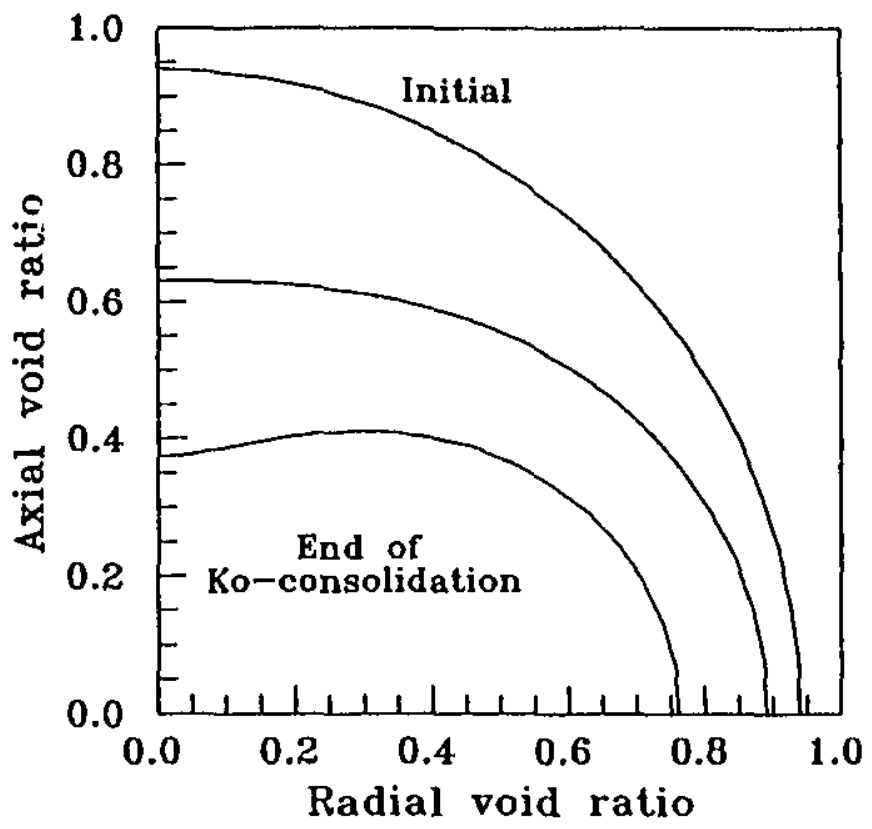

Figure 4.6b Evolution of spatial distribution of void ratio; numerical simulation based on equation (2.24). 


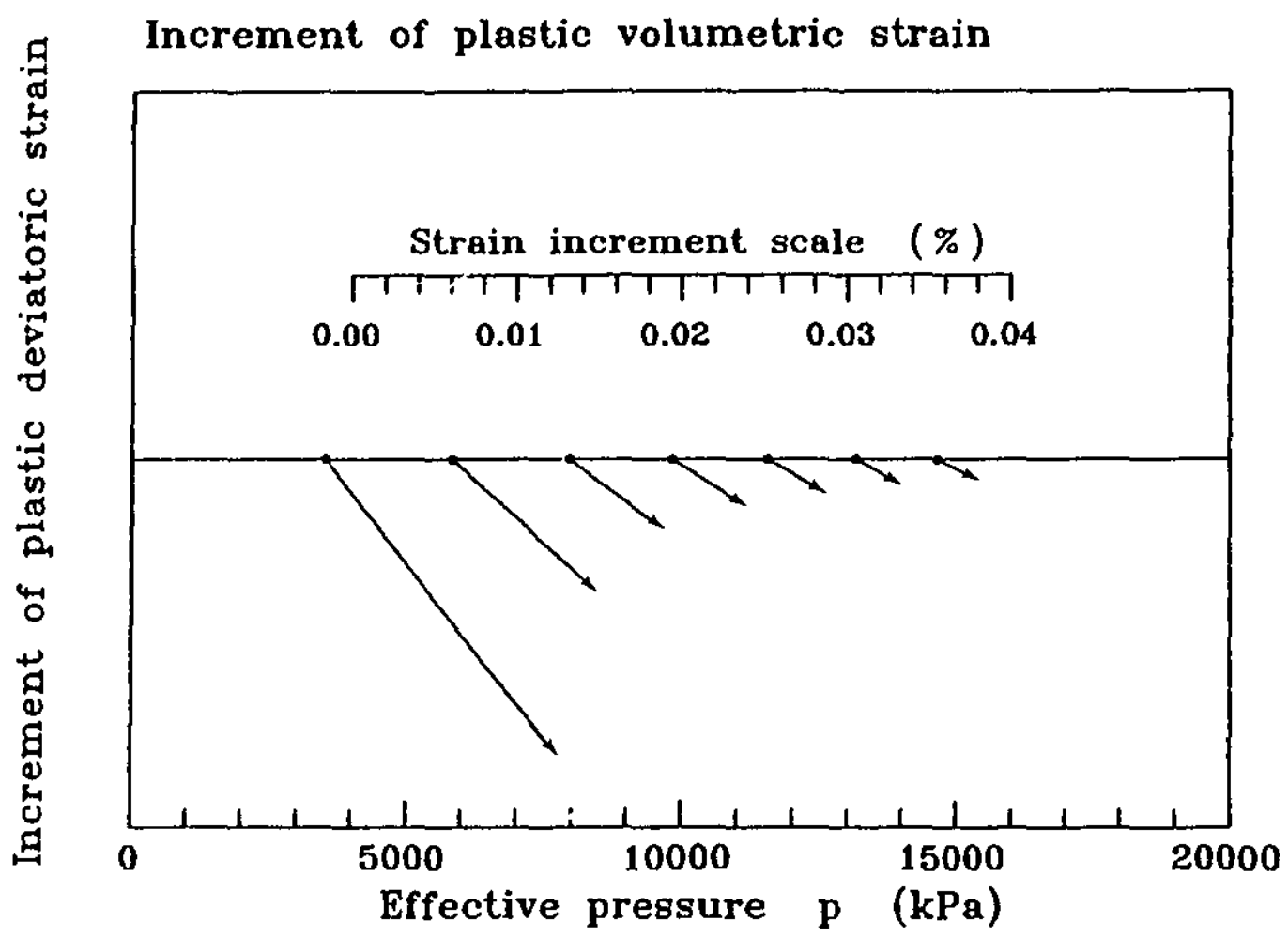

Figure 4.7a Numerical simulation of hypotetical response during hydrostatic compression following Ko-consolidation and undrained unloading. 


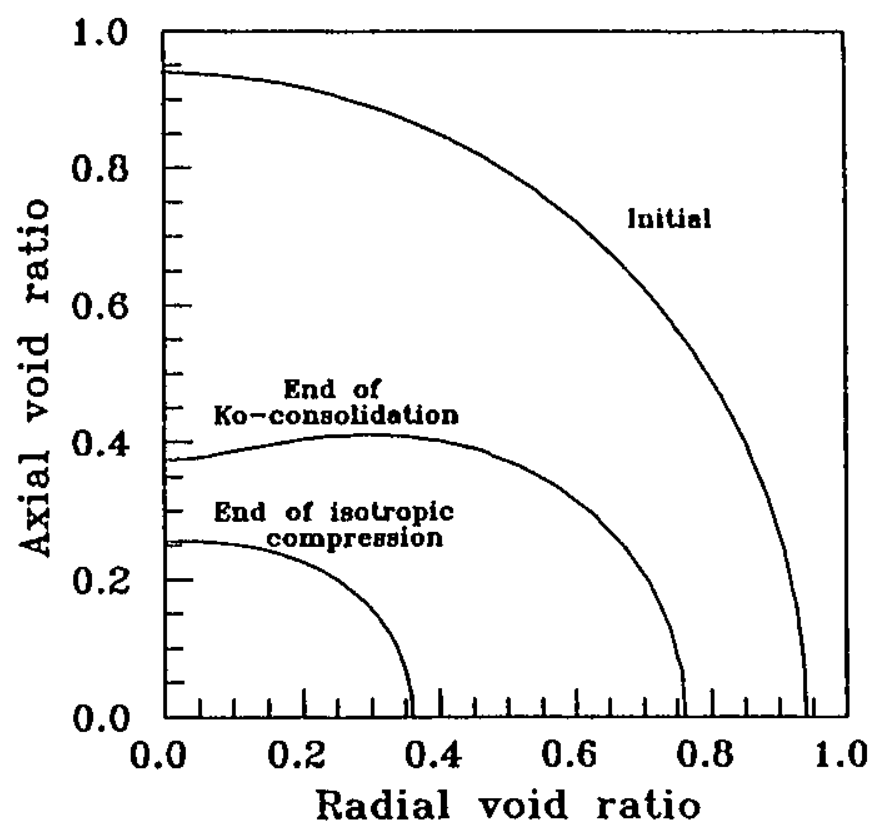

Figure $4.7 \mathrm{~b}$ Evolution of spatial distribution of void ratio; numerical simulation based on equation (2.24). 

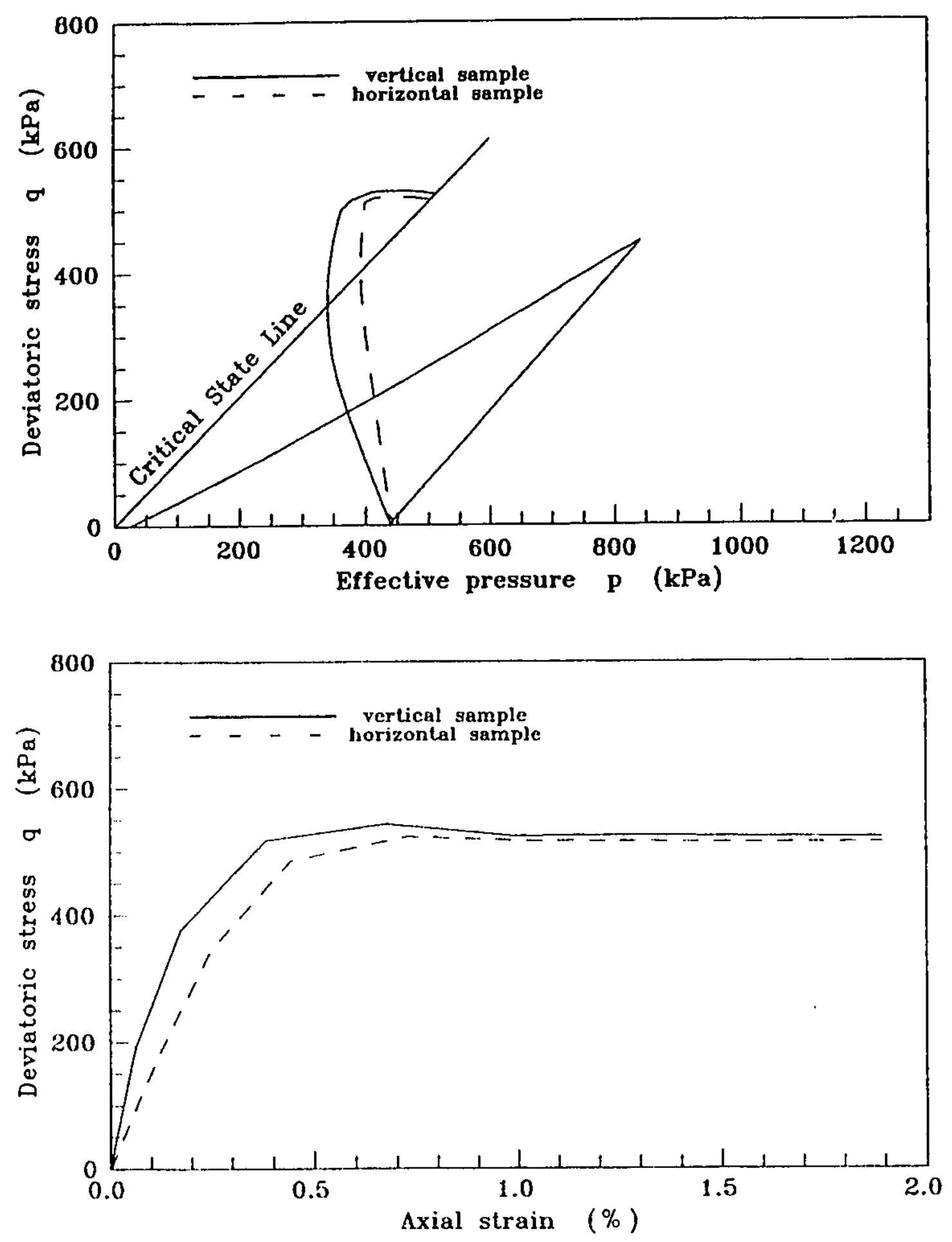

Figure 4.8 Undrained compression following Ko-consolidation and unloading; (a) stress paths, (b) material characteristics. 


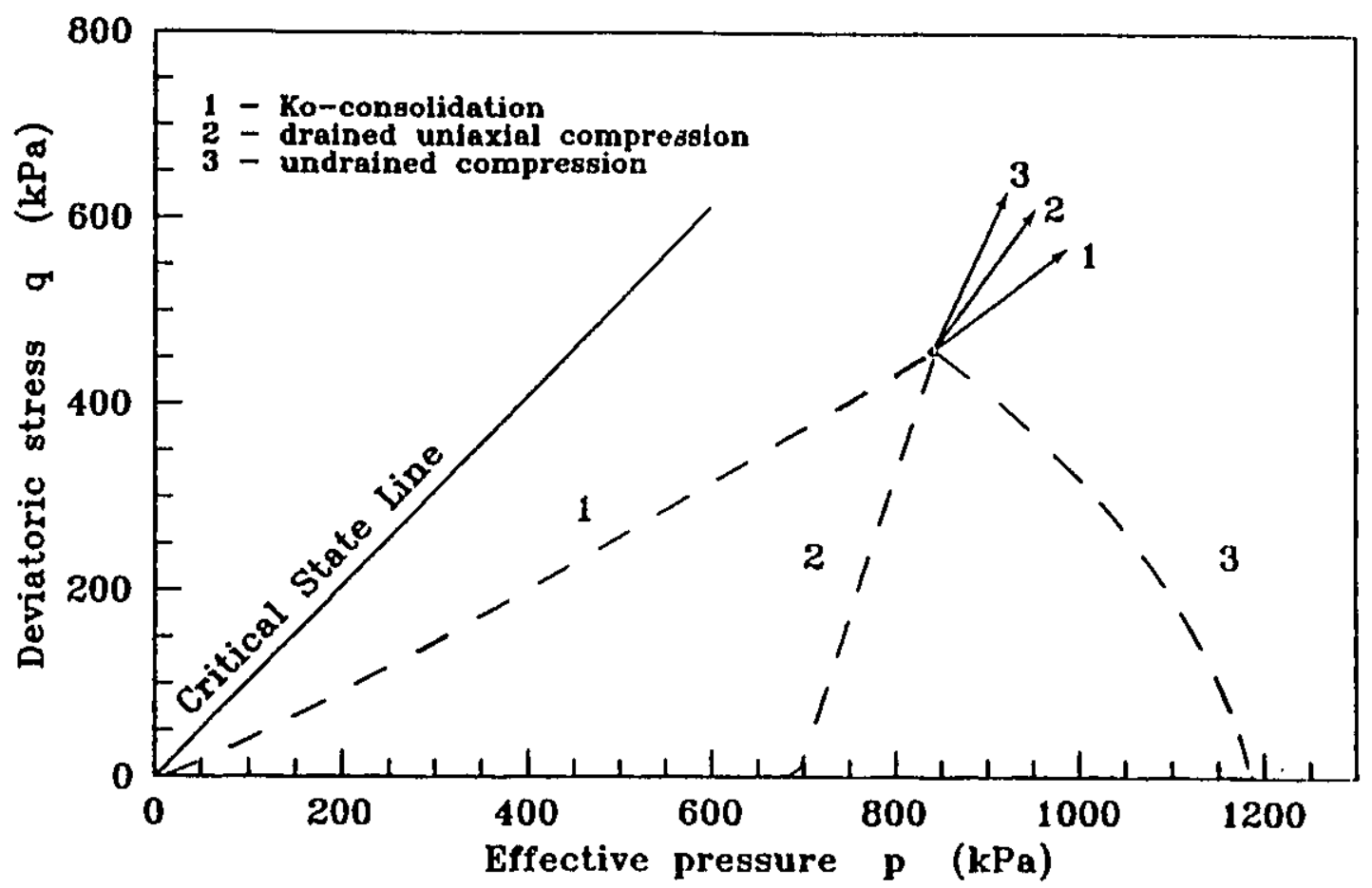

Figure 4.9 Stress probe experiment; direction of plastic strain increment. 


\section{CHAPTER 5}

\section{CONSIDERATIONS ON SOIL RESPONSE TO THE ROTATION OF PRINCIPAL STRESS DIRECTIONS}

\subsection{Introduction.}

In many practical geotechnical situations, the principal stress directions, in the vicinity of an engineering structure, deviate from those imposed by the depositional conditions. This may have a pronounced effect on the resulting displacement field. In recent years a number of researchers (Miura, 1986; Ishihara, 1983) performed material tests which invariably indicate the sensitivity of soil response to the rotation of principal stress axes. Although the experimental information is still fragmentary, the evidence gathered so far is very convincing (at least in a qualitative sense) and should not be ignored when formulating appropriate constitutive relations.

Over the last two decades a large number of phenomenological models for geomaterials have been proposed (Desai and Siriwardane, 1984; Pietruszczak and Pande, eds., 1989). These models are mostly cast in the framework of theory 
of plasticity and they do not, in general, account for the influence of the rotation of principal stress axes. In order to demonstrate this, let us examine briefly the mathematical structure of the existing, well established formulations.

5.2 Theoretical considerations.

Consider an arbitrary loading history confined to pure rotation of the principal stress system. In other words, assume that a sample, under initial stress $\sigma^{0}{ }_{i j}$, is subjected to the stress increment

$$
\Delta \sigma_{i j}=T_{i p} T_{j q} \sigma_{p q}^{0}-\sigma_{i j}^{0}
$$

Here, $\Delta \sigma_{i j}$ and $\sigma^{0}{ }_{i j}$ are referred to the same Cartesian coordinate system fixed within the material and $\mathrm{T}_{\mathrm{ij}}$ is the usual transformation tensor. The trajectory (5.1), when imposed on a sample, results in the deviation of principal stress axes from those corresponding to $\sigma^{0}{ }_{i j}$, while the principal values of $\sigma^{0}{ }_{i j}$ are preserved. Suppose at first, that the material is isotropic, linearly elastic. In this case, the Hooke's law takes the form

$$
\Delta \epsilon_{i j}=\Delta \sigma_{i j} / 3 K \quad ; \quad \Delta e_{i j}=\Delta S_{i j} / 2 G
$$


where $s_{i j}$ and $e_{i j}$ represent the deviatoric parts of the stress and strain tensor and $\mathrm{K}, \mathrm{G}$ are the elastic constants (i.e. the bulk and the shear modulus, respectively). The orthogonality conditions imposed on the direction cosines $T_{i j}$, i.e.,

$$
\mathrm{T}_{\mathrm{ip}} \mathrm{T}_{\mathrm{iq}}=\delta_{\mathrm{pq}}
$$

constrain the stress path (5.1) to satisfy

$$
\Delta \sigma_{i \mathrm{i}}=0 ; \quad \Delta \mathrm{s}_{\mathrm{ij}}=\Delta \sigma_{\mathrm{ij}}
$$

so that, according to equations (5.2)

$$
\Delta \epsilon_{i j}=0 ; \quad \Delta e_{i j}=\frac{1}{2 G}\left(T_{i p} T_{j q}-\delta_{i p} \delta_{j q}\right) \sigma_{p q}^{0}
$$

Thus, the imposed path results in generation of deviatoric strain $e_{i j}$, while the volume change remains zero. It should be noted now, that the existing experimental cvidence (Miura, 1986; Ishihara and Towhata, 1983) clearly indicates that the trajectories (5.1) induce not only irreversible but also permanent deformation of both distortional as well as volumetric nature.

Let us examine now, in a similar context, the formulations derived from the theory of plasticity. The simplest of all, are the descriptions admitting isotropic 
hardening. In the most common approach, the evolution of the yield surface, $f=0$, is linked to generation of irreversible volumetric strain, $\epsilon_{i 1}^{p}$, so that

$$
f\left(\sigma_{i j}, \epsilon_{i j}^{p}\right)=0 \quad ; \quad \dot{\epsilon}_{i j}^{p}=\dot{\lambda} \frac{\partial f}{\partial \sigma_{i j}}
$$

In such case, the consistency condition

$$
\dot{\mathrm{f}}=\frac{\partial \mathrm{f}}{\partial \sigma_{i j}} \dot{\sigma}_{i j}+\frac{\partial f}{\partial \epsilon^{\mathrm{p}}{ }_{i i}} \dot{\epsilon}_{k k}=0
$$

results in

$$
\dot{\lambda}=\frac{1}{H}\left(\frac{\partial \mathrm{f}}{\partial \sigma_{i j}} \dot{\sigma}_{i j}\right) \quad ; \quad \mathrm{H}=-\frac{\partial \mathrm{f}}{\partial \epsilon^{\mathrm{p}}{ }_{i \mathrm{i}}} \frac{\partial \mathrm{f}}{\partial \sigma_{\mathrm{kk}}}
$$

Since $f=0$ is an isotropic function of $\sigma_{i j}$, then for all stress histories restricted to pure rotation of principal stress axes, equation (5.1), there is

$$
\frac{\partial \mathrm{f}}{\partial \sigma_{i j}} \dot{\sigma}_{i j}=0 \rightarrow \dot{\lambda}=0
$$

i.e. the loading process is a 'neutral' one and does not produce any plastic deformations.

The same conclusion can also be reached when employing multi-yicld loci theories of soil deformation. In this case, the associated flow rule reads 


$$
\dot{\epsilon}_{i j}^{p}=\sum_{n} \dot{\lambda}_{n} \frac{\partial f_{n}}{\partial \sigma_{i j}}
$$

where the summation includes only those terms which correspond to $f_{n}=0$ and $f_{n}=0$. Let $f_{m} \in f_{n}(n=1,2 \ldots N)$ be expressed in a form

$$
f\left(\sigma_{i j}, \epsilon_{i i}^{p}, \epsilon^{p}\right)=0
$$

where

$$
\dot{\epsilon}^{p}=\left(e^{p}{ }_{i j} e^{p}{ }_{i j}\right)^{1 / 2} ; \quad \dot{e}^{p}{ }_{i j}=\dot{\epsilon}^{p}{ }_{i j}-1 / 3 \delta_{i j} \dot{\epsilon}_{k k}^{p}
$$

The consistency condition for any $\mathrm{f}_{\mathrm{m}}=0$ takes the form

$\dot{f}=\frac{\partial f}{\partial \sigma_{i j}} \sigma_{i j}+\sum_{n} \dot{\lambda}_{n}\left[\frac{\partial f_{m}}{\partial \epsilon_{i i}^{p}} \frac{\partial f_{n}}{\partial \sigma_{k k}} \frac{\partial f_{m}}{\partial \epsilon^{p}}\left(\frac{\partial f_{n}}{\partial \sigma_{(i j)}} \frac{\partial f_{n}}{\partial \sigma_{(i j)}}\right)^{t}\right]=0$

where $\partial f / \partial \sigma_{(i j)}$ designates the deviatoric part of the gradient tensor.

Denoting $\left(\partial f_{m} / \partial \sigma_{i j}\right) \dot{\sigma}_{i j}$ by $\underline{A}_{m}$ and the term contained in square brackets by $\mu_{m}$, a set of simultaneous equations (5.8) can be rewritten in the form

$$
\left\{\dot{\lambda}_{n}\right\}=\left[\mu_{m}\right]^{-1}\left\{A_{m}\right\}
$$


It is obvious that if $\left\{\dot{\hat{A}}_{m}\right\}=\{0\}$ then, for arbitrary $\left[\mu_{m n}\right]$, there must be $\left\{\dot{\lambda}_{n}\right\}=0$. In typical formulations employing kinematic hardening, the yield surface undergoes translation guided by a tensorial argument $\alpha_{i j}$, i.e.

$$
f=f\left(\sigma_{i j}, \alpha_{i j}, \epsilon_{i i}^{p}\right)=0
$$

where $\alpha_{i j}=\alpha_{i j}\left(\epsilon_{k l}^{p}\right)$, i.e. the evolution of the yield surface is implied by generation of plastic deformations. According to equation (5.14) the consistency condition becomes

$$
\dot{\mathrm{f}}=\frac{\partial \mathrm{f}}{\partial \sigma_{i j}} \dot{\sigma}_{i j}+\frac{\partial \mathrm{f}}{\partial \alpha_{i j}} \frac{\partial \alpha_{i j}}{\partial \epsilon_{k l}^{\mathrm{p}}} \dot{\epsilon}_{k l}^{\mathrm{p}}+\frac{\partial \mathrm{f}}{\partial \epsilon_{i j}^{\mathrm{p}}} \dot{\epsilon}_{k k}^{\mathrm{p}}=0
$$

and results in a similar functional form to that of equation (5.8), i.e.

$$
\begin{aligned}
& \dot{i}=\frac{1}{H}\left(\frac{\partial f}{\partial \sigma_{i j}} \dot{\sigma}_{i j}\right) \\
& H=-\frac{\partial f}{\partial \epsilon_{i i}^{p}} \frac{\partial f}{\partial \sigma_{k k}}-\frac{\partial f}{\partial \alpha_{i j}} \frac{\partial \alpha_{i j}}{\partial \epsilon_{k l}^{p}} \frac{\partial f}{\partial \sigma_{k l}}
\end{aligned}
$$

In this case again, pure rotation of principal stress directions describes a 'neutral' process, for which $\dot{\lambda}=0$ and thus $\dot{\epsilon}_{i j}^{p}=0$. 
Finally, another common description is that of 'bounding surface plasticity' (Dafalias, 1986; Pietruszczak and Mroz, 1983). Here, for stress histories penetrating the interior of the bounding surface, the deformation process is described in terms of evolution of an inner loading surface

$$
f_{l}\left(\sigma_{i j}, \alpha_{i j}\right)=0
$$

The kinematics of this surface (i.e. direction of translation) is guided by a 'conjugate' stı ess tensor $\sigma^{c}{ }_{i j}$ located on the bounding surface. A typical form of the evolution law for the components of $\alpha_{i j}$ is

$$
\dot{\alpha}_{i j}=\dot{\mu} B_{i j} \quad ; \quad B_{i j}=B_{i j}\left(\sigma_{k l}, \sigma_{k !}^{c}\right)
$$

where $\mu$ is a scalar parameter, determined via consistency condition

$$
\dot{f}_{l}=\frac{\partial f_{l}}{\partial \sigma_{: j}} \dot{\sigma}_{i j}+\frac{\partial f_{l}}{\partial \alpha_{i j}} \beta_{i j} \dot{\mu}=0
$$

According to equation (5.19), for $\left(\partial f_{l} / \partial \sigma_{i j}\right) \dot{\sigma}_{i j}$ there is $\dot{\mu}=0$ and the surface $\mathrm{f}_{\mathrm{t}}=0$ remains stationary. Therefore, once again, the formulation does not respond to the rotation of principal stress directions. 
It is evident from the above considerations, that none of the existing frameworks, based on classical concepts of isotropic/kinematic hardening, is capable of taking into account the sensitivity of plastic flow to the rotation of principal stress axes. Indeed, if the evolution of the yield surface (regardless of its form) results exclusively from the generation of plastic deformations, then these effects can never be properly embraced.

There are several ways of enhancing the plasticity framework so that it responds to a change in orientation of the principal stress system. This has been accomplished, for example, in formulation proposed by Pande and Sharma (1983), (see also Pietruszczak and Pande; 1987) wherehy the macroscopic behaviour of a sample has been deduced from deformation pattern along an infinite number of randomly oriented planes, by using an appropriate averaging procedure. An alternative approach is that derived in Chapter 3, which incorporates new tensorial function(s) capable of representing the soil fabric and its evolution. In what follows, the framework outlined in Chapter 3 is reviewed in the context of its ability to describe the sensitivity of soil response to the rotation of principal stress axes.

Examine first the formulation defined by the equations (3.22) and (3.23), which assumes that $\Omega_{i j}$ affects directly the functional form of the yield criterion, i.e.,

$$
f=f\left(\sigma_{i j}, \Omega_{i j}, æ\right)=0
$$


In such circumstance, $f=0$ depends on the deformation history (recorded by $æ$ ) and, in general, ten functionally independent invariants of both tensors $\underline{\sigma}$ and $\underline{\Omega}$. Now, it is interesting to note that the yield surface, defined by equation (5.20), will undergo progressive evolution not only during an active loading process, but for all histories (including those for which $\mathrm{f}<0$ ) associated with the rebuilding of material microstructure. Consequently, such a formulation will account for the influence of the rotation of principal stress axes. In order to demonstrate this, let us assume again $æ=\epsilon_{i i}^{\mathrm{P}}$ and write the consistency condition in the form

$$
\dot{\mathrm{f}}=\frac{\partial \mathrm{f}}{\partial \sigma_{i j}} \dot{\sigma}_{i j}+\frac{\partial \mathrm{f}}{\partial \Omega_{i j}} \dot{\Omega}_{i j}+\frac{\partial \mathrm{f}}{\partial \epsilon_{i i}^{\mathrm{p}}} \dot{\epsilon}_{\mathrm{kk}}^{\mathrm{p}}=0
$$

Utilizing the evolution law (2.24) for the components of $\Omega_{\mathrm{ij}}$, i.e.

$$
\dot{\Omega}_{i j}=B \dot{e}^{e}{ }_{i j}+B \dot{e}^{p}{ }_{i j}=\frac{B}{2 G} \dot{s}_{i j}+B \dot{\lambda} \frac{\partial f}{\partial \sigma_{(i j)}}
$$

and substituting the above representation back in equation (5.21), one obtains

$$
\begin{aligned}
& \dot{i}=\frac{1}{H}\left(\frac{\partial f}{\partial \sigma_{i j}}+\frac{B}{2 G} \frac{\partial f}{\partial \Omega_{i j}}\right) \dot{\sigma}_{i j} \\
& H=-\frac{\partial f}{\partial \epsilon_{i j}^{p}} \frac{\partial f}{\partial \sigma_{k k}}-\frac{\partial f}{\partial \Omega_{i j}} \frac{\partial f}{\partial \sigma_{(i j)}}
\end{aligned}
$$


It is evident from equations (5.23) and (5.24) that for $\left(\partial \mathrm{f} / \partial \sigma_{i j}\right) \dot{\sigma}_{i \mathrm{j}}=0$ there is

$$
\dot{\epsilon}_{i j}^{p}=C_{i j k l} \dot{\sigma}_{k l} \quad ; \quad C_{i j k l}=\frac{B}{2 G H} \frac{\partial f}{\partial \sigma_{i j}} \frac{\partial f}{\partial \Omega_{k l}}
$$

which implies that for histories experiencing pure rotation of principal stress axes, the progressive generation of plastic strain takes place, provided that $\left(\partial f / \partial \Omega_{i j}\right) \dot{\sigma}_{i j}>0$.

As pointed out in section 3.3, a simpler description than that based on equation (5.20) may be obtained by resolving the plastic strain rate into components governing a pseudo-isotropic response and refraction from it. Such a flow rule is expressed by equations (3.24), i.e.,

$$
\dot{\epsilon}_{i j}^{\mathrm{p}}=\dot{\lambda} \frac{\partial \mathrm{f}(\underline{\sigma}, \mathfrak{x})}{\partial \sigma_{i j}}+\dot{\mu} \mathrm{G}_{\mathrm{ij}}(\underline{\Omega}, \mathfrak{x})
$$

In particular, as discussed in section 3.3 , one may simply take

$$
\dot{\epsilon}_{i j}^{p}=\dot{\lambda}\left(\frac{\partial f}{\partial \sigma_{i j}}+h \Omega_{i j}\right)
$$

Although the formulation (5.27) is very attractive, in the sense of its numerical simplicity and performance, it does not respond to the rotaiion of principal stress 
axes. Indeed, when $æ$ is identified with $\epsilon_{i j}^{\mathfrak{p}}$, i.e. plastic volumetric strain generated in a fictitious isotropic medium, then the consistency condition (3.32) results in a similar functional form to that of equation (5.7)

$$
\dot{\lambda}=\frac{1}{\mathrm{H}}\left(\frac{\partial \mathrm{f}}{\partial \sigma_{\mathrm{ij}}} \dot{\sigma}_{\mathrm{ij}}\right) \quad ; \quad \mathrm{H}=-\frac{\partial \mathrm{f}}{\partial \epsilon_{\mathrm{ij}}^{\mathrm{p}}} \frac{\partial \mathrm{f}}{\partial \sigma_{\mathrm{kk}}}
$$

It appears that the only rational way of enhancing the representation (5.27), is to define the condition of the neutral state relative to the actual direction of plastic flow, i.e.,

$$
i=\frac{1}{H}\left(\frac{\partial f}{\partial \sigma_{i j}}+h \Omega_{i j}\right) \dot{\sigma}_{i j}
$$

In the latter case, it is evident that the stress trajectories resulting from pure rotation of principal stress axes will generate the plastic strain rates

$$
\dot{\epsilon}_{i j}^{p}=C_{i j k l} \dot{\sigma}_{k l} \quad ; \quad C_{i j k l}=\frac{h}{H}\left(\frac{\partial f}{\partial \sigma_{i j}}+h \Omega_{i j}\right) \Omega_{k l}
$$

provided that $\Omega_{i j} \dot{\sigma}_{i j}>0$.

Finally, let us examine the formulation given by equation (5.27), i.e. that obtained by admitting a coupling between both terms in equation (5.26), 


$$
\dot{\epsilon}_{i j}^{p}=\dot{i} \frac{\partial f}{\partial \sigma_{i j}}+h C_{i j k l}\left(\frac{\partial g(\underline{\underline{\Omega}})}{\partial \bar{\Omega}_{k l}} \frac{\partial f_{q}(\underline{\underline{\Omega}})}{\partial \bar{\Omega}_{p q}}\right) \dot{\bar{\Omega}}_{p q}
$$

By inspecting equation (5.31), it is again obvious that this framework is capable of responding to pure rotation of the principal stress system. Under $\left(\partial f / \partial \sigma_{i j}\right) \dot{\sigma}_{i j}=0$, one obtains the contribution from the second term in equation (5.31). Hence, the stress trajectories resulting from pure rotation of principal stress axes will generate the plastic strain rates

$$
\dot{\epsilon}_{i j}^{p}=h C_{i j k l}^{e}\left(\frac{\partial g(\underline{\underline{\Omega}})}{\partial \bar{\Omega}_{k l}} \frac{\partial f_{1}(\underline{\underline{\Omega}})}{\partial \bar{\Omega}_{p q}}\right) \dot{\bar{\Omega}}_{p q}
$$

provided that,

$$
\dot{\mu}=\frac{\partial \mathrm{f}_{1}(\underline{\bar{\Omega}})}{\partial \bar{\Omega}_{\mathrm{ij}}} \dot{\bar{\Omega}}_{\mathrm{ij}}>0
$$


5.3 Numerical simulations of tests with the rotation of principal stress directions.

The ability of the proposed plasticity formulation to model the influence of the rotation of principal stress axes is now illustrated by some numerical examples. The simulations are based on equation (5.27) with the condition of neutral state defined by equation (5.29). The yield criterion is again specified by the functional form (4.10). The quantitative aspects are, at this stage, rather speculative, as no experimental information pertaining to clays is available. All simulations relate to normally consolidated clays, under $\mathrm{K}_{0}$-condition. The material is assumed to be initially isotropic and is described by the set of material parameters specified in section 4.4.1.

The results shown in Figure 5.1 correspond to a specimen which has been consolidated, under $\mathrm{K}_{0}$ condition, to $\sigma_{v}^{\prime}=1040 \mathrm{kPa}$ and subsequently subjected to a progressive rotation of principal stress directions, from $0^{\circ}$ to $180^{\circ}$. Figure $5.1 \mathrm{a}$ shows the history of the components of elastic strain. In the elastic range the response is governed by equation (5.2), thus the imposed stress trajectory results in generation of reversible deviatoric strain only. At the same time, the plastic flow contributes to both distortional as well as to volumetric strain, as clearly indicated in Figure 5.1b. It should be mentioned that the only experimental information available, for a similar load configuration, is that pertaining to sand e.g. Miura and Shosuke (1986). The predicted deformation history seem to be in qualitative 
agreement with reported results and can not be predicted by most of plasticity models for geomaterials. Figure 5.2 complements Figure 5.1 and shows the evolution of the spatial distribution of void ratio during both $\mathrm{K}_{0}$-consolidation and subsequent rotation of principal stress axes. The induced anisotropy is displayed throughout a significant bias in the spatial distribution of voids.

Figure 5.3. presents a hypothetical response of $\mathrm{K}_{0}$-consolidated sample during undrained uniaxial compression combined with a continuous rotation of principal stress axes. It is evident that the evolution of material structure, which is induced by different rates of rotation, results in the non-coincidence of effective stress paths. Consequently, the ultimate strength of the material is reached at different levels of effective stresses. The evolution of the spatial distribution of void ratio associated with the entire loading programme, is shown in Figure 5.3.b. The rotation of the principal stress directions, results in the deviation of principal axes of tensor $\underline{\Omega}$ from the coordinate system fixed in the sample.

Finally, Figure 5.4 presents the influence of the rate of the rotation of principal stress axes on the deviatoric characteristics. The loading process results in progressive reduction in ultimate strength as the amount of rotation increases. 


\subsection{Concluding remarks.}

Concluding, the proposed constitutive relations are capable of describing the influence of the inherent as well as induced anisotropy on the deformation process. Their mathematical structure permits, in general, the modelling of the sensitivity of soil response to the rotation of principal stress axes. The possibility of modelling of these effects is essential from the view point of practical engineering applications. 

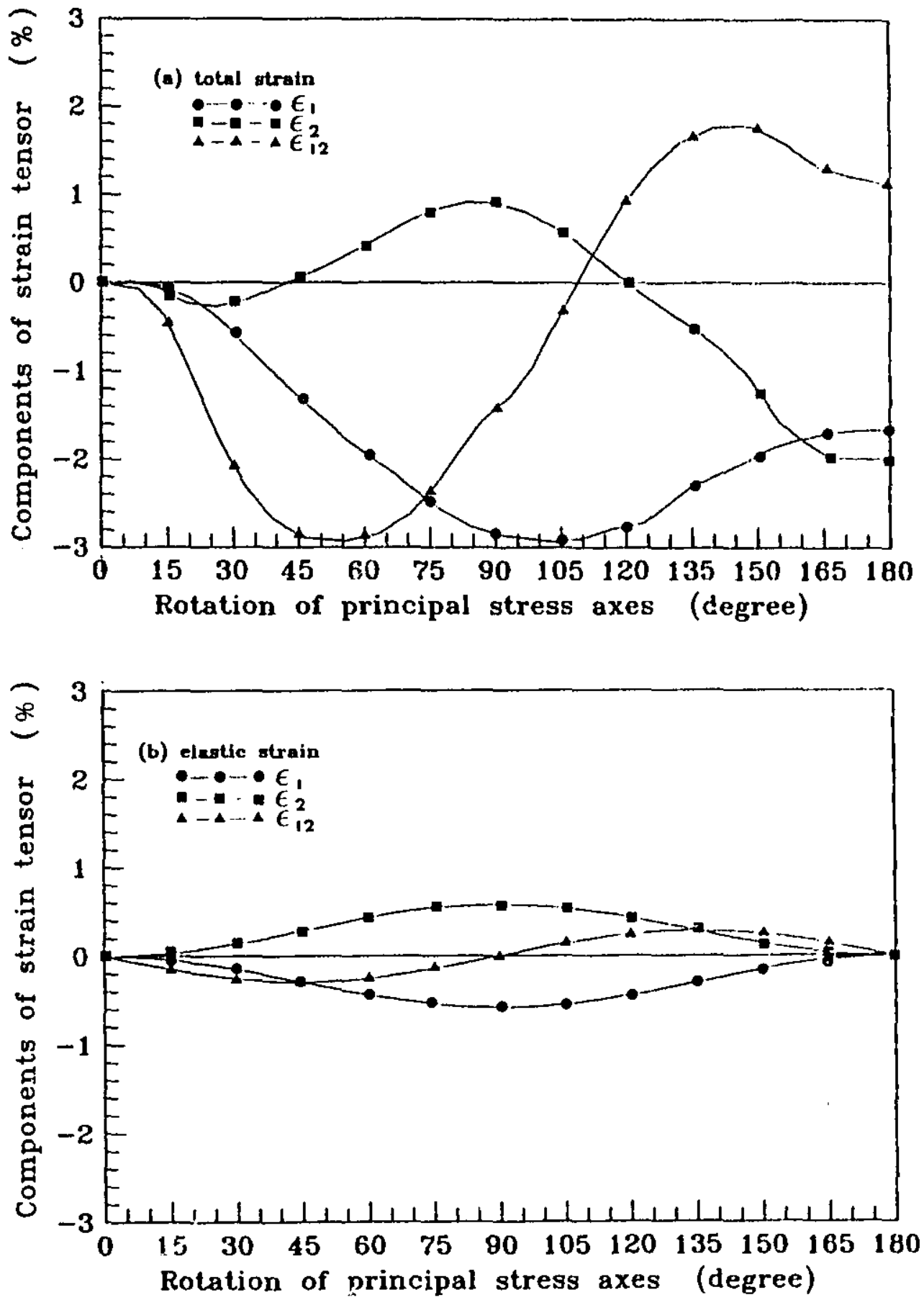

Figure 5.1 Total and elastic strains generated by the pure rotation of the principal stress system following Ko-consolidation; (a) total strain, (b) elastic strain 


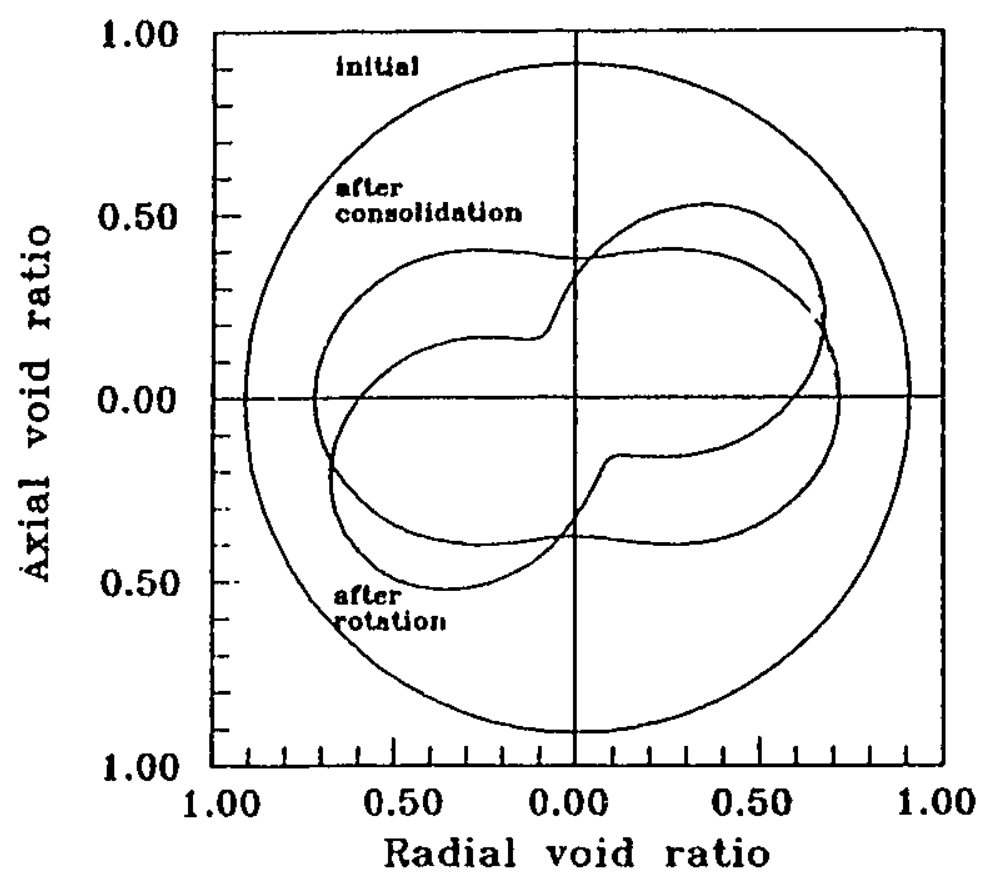

Figure 5.2 Evolution of spatial distribution of void ratio under pure rotation of the principal stress system. 

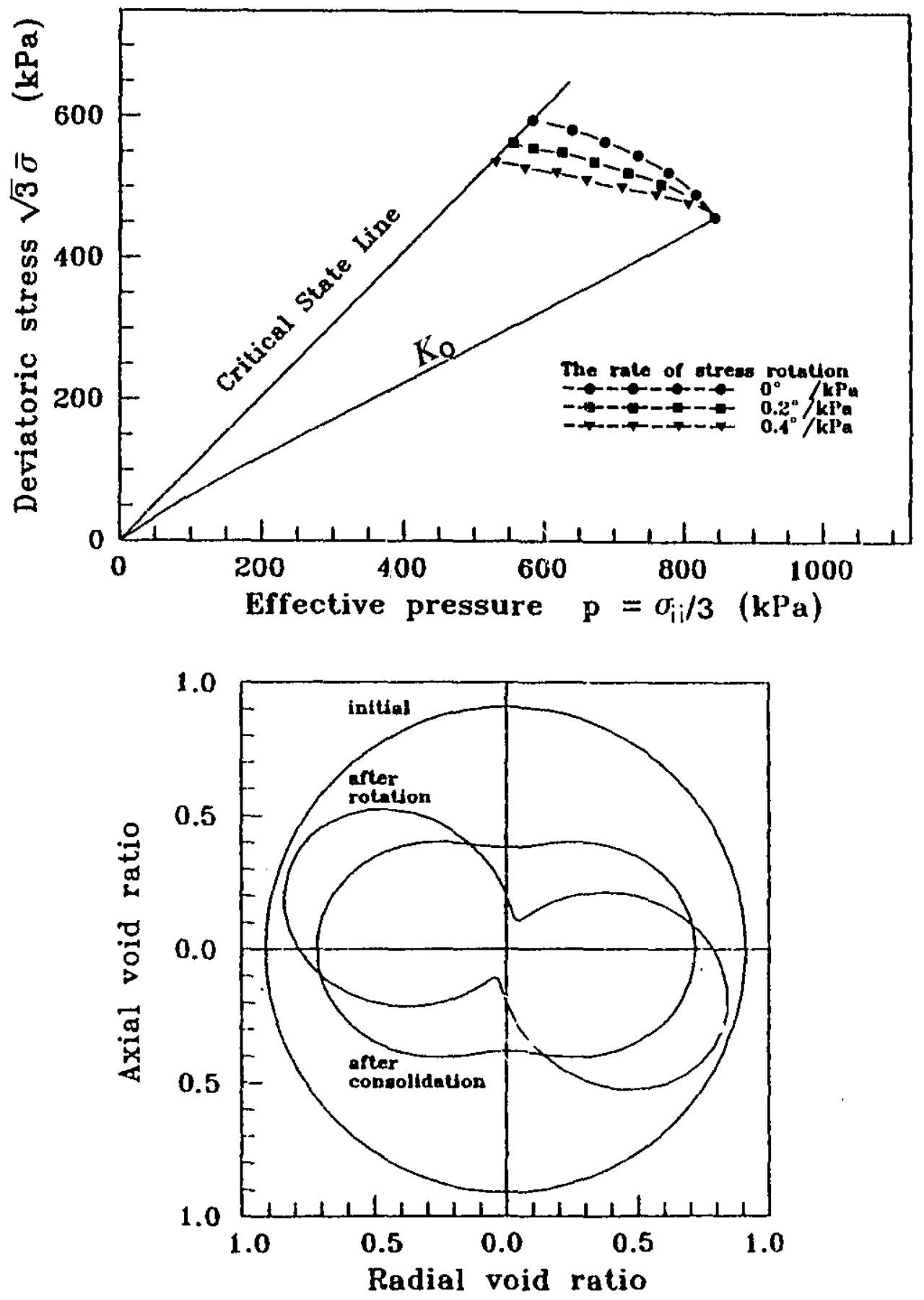

Figure 5.3 Uniaxial undrained compression of Ko-consolidated specimens with diffrent rates of the rotation of principal stress axes; (a) stress paths, (b) evolution of spatial distribution of void ratio at the rate of $0.2^{\circ} / \mathrm{kPa}$. 


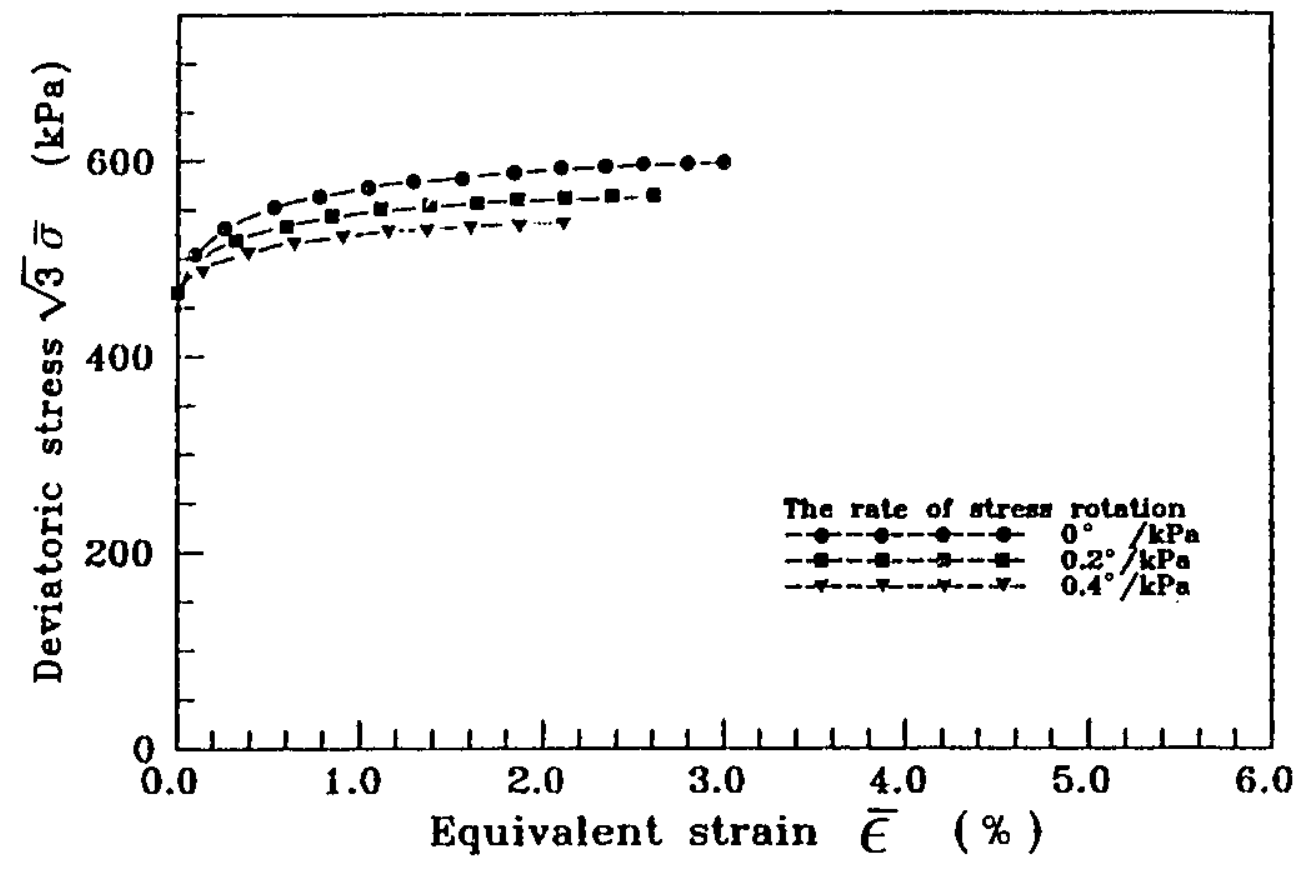

Figure 5.4 Stress-strain response during undrained compression combined with a continous rotation of principal stress axes. 


\section{CHAPTER 6}

\section{FINAL REMARKS AND RECOMMENDATIONS}

\subsection{Summary and Conclusions}

In recent years, the research in geotechnical area has been mainly focused on advances in the testing techniques and instrumentation, as well as on the development of new constitutive relations. The modelling of gec :echnical problems usually involves the use of complex approaches of modern physics and applied mathematics, as soils are multi-phase materials with strong nonlinear and history-dependent mechanical behaviour. On the other hand, the practising geotechnical engineer is often in the situation where, in a limited period of time, he is expected to provide a solution to a real structural problem with the specified amount of confidence. It is natural that in his endeavour to predict the unknown, he is often conservative in his judgment and sceptical about theoretical solutions which are not confirmed by case histories. Such a view point is rational, as in most cases the excessive costs due to a conservative design are still incomparably smaller than those resulting from a necessity of improving an erected structure which was improperly designed. 
The engineering design can be characterized as the process of progressive data reduction; the data from both the field and laboratory investigations are processed with the help of continuum mechanics and applied mathematics. In such a process the understanding of physical behaviour of soil under anticipated loads and conditions is essential. Thus, the fundamental investigations, aimed at mathematical description of soil deformation, are very meritorious. On one hand, they serve the purpose of an improved and economized design, on the other one they eventually allow to approach complex structural problems which can not be solved by conventional design procedures.

In this thesis an attempt has been made to bridge the gap between the knowledge accumulated in the areas of constitutive modelling and soil physics. The developed framework incorporates some aspects of soil physics, investigated at micro-level, by introducing the concept of fabric tensor. Such an approach leads to constitutive relations which are capable to model the effects of inherent and induced anisotropy in particulate media. In the proposed formulation the fabric has been implicitly described by some continuous measures reflecting the spatial distribution of phase composition of the material. It is clear from numerical simulations presented in Chapters 4 and 5 that such a formulation is advantageous over a conventional plasticity approach. The incorporation of the proposed modifications into the framework of Critical State Theory results in a constitutive law that can describe, at least in a qualitative manner, various manifestations of clay anisotropy. It should be emphasised that this goal was achieved without deliberate geometrical 
assumptions concerning the yield function or the plastic potential, and using a quite unsophisticated model, i.e., the density hardening model.

The presented mathematical framework is general and can be extended to other geological materials. In order to achieve this, the evolution law, equation (2.24), as well as the selected the material functions ought to be consistently modified for each specific material. The identification of the oriented fabric is not a simple matter and it calls for new experimental techniques. In its present version, the model does not take into account the irreversibility of plastic flow and the evolution microstructure during stress reversals. However, the mathematical structure of governing equations allows to include these effects in future developments.

6.2 Recommendations for Future Research.

Despite a significant amount of interest devoted to the microscopic studies of soils and the development of phenomonological models with structure, until now no consistent programme has been developed combining the experimental research with analytical efforts. For future research activities it is recommended to design an experimental programme in which both the macroscopic mechanical behaviour and the evolution of phase distribution due to microstructural changes 
could be correlated. This would improve the understanding of the physical aspects of the problem and permit a proper identification of the evolution law for the fabric tensor in a wide range of loading domains. Hopefully, such a programm would also allow to define the minimum number of tests for selection of employed parameters in the domain of applicability of the model.

In the subsequent analytical development, additional studies are required in order to embody the effects of cycling loading. Moreover, the attention should also be paid to sensitivity of the model with respect to variations of the material parameters. Finally, as the process of fabric changes is time-dependent, the extension of the proposed concept to visco-plasticity can offer further improvements in mathematical descriptions of clay behaviour. 


\section{APPENDIX A}

\section{DETERMINATION OF GRADIENT TENSOR}

In order to implement the constitutive relations (3.39) and (3.55) the appropriate gradient tensors of the yield function (4.10) should be specified. In the stress reference frame $I, \bar{\sigma}, \theta$, the gradient of the yitud function can be written in the following from (cf. section 4.2)

$$
\frac{\partial f}{\partial \sigma_{i j}}=C_{1} \underline{b}_{1}+C_{2} \underline{b}_{2}+C_{3} \underline{b}_{3}
$$

Three scalars $C_{1}, C_{2}, C_{3}$ appearing in the above equation depend on the form of the yield function. For the function described by equation (4.10), these scalars are specified as follows:

for the parabolic segment of function, i.e., $\left(0<I \leq I_{x}\right)$;

$$
\begin{aligned}
& C_{1}=\frac{2 \eta g(\theta)\left(I_{0}-I_{x}\right)\left(I-I_{x}\right)}{I_{x}{ }^{2}} \\
& C_{2}=\sqrt{3}
\end{aligned}
$$




$$
C_{3}=-\frac{\sqrt{3} \bar{\sigma}}{g(\theta)} \frac{\partial g(\theta)}{\partial \theta}
$$

for the elliptic segment of the function, i.e. $\left(I_{x} \leq I \leq I_{0}\right)$;

$$
\begin{aligned}
& C_{1}=2\left(I-I_{x}\right) \\
& C_{2}=\frac{6 \bar{\sigma}}{(\eta g(\theta))^{2}} \\
& C_{3}=-\frac{6 \bar{\sigma}^{2}}{\eta^{2} g^{3}(\theta)} \frac{\partial g(\theta)}{\partial \theta}
\end{aligned}
$$

where,

$$
\frac{\partial g(\theta)}{\partial \theta}=\frac{3 \mathrm{a}(1-\mathrm{K}) \mathrm{g}^{2}(\theta) \cos 3 \theta}{2 \mathrm{~K}(\sqrt{1+\mathrm{a}}-\sqrt{1-\mathrm{a}}) \sqrt{1-\mathrm{a} \sin 3 \theta}}
$$

Moreover, if the deformation history of soil is recorded by $\epsilon^{p_{i j}}$, i.e., plastic volumetric strain generated in a fictitious isotropic medium, then

$$
\frac{\partial \mathrm{f}}{\partial \epsilon_{i i}^{\mathrm{p}}}=-\frac{\left(1+\mathrm{e}_{0}\right)}{(\lambda-\kappa)} \frac{\eta \eta_{f}}{\left(\eta+\eta_{f}\right)}\left(\frac{\mathrm{I}}{\mathrm{I}_{x}}\right)^{2}
$$

The derivatives $\left(\underline{\mathbf{b}}_{1}, \underline{\mathbf{b}}_{2}, \underline{\mathbf{b}}_{3}\right)$ are intrpreted as vectors in a six-dimensional effective stress space and are defined by the following expressions:

$$
\underline{\mathbf{b}}_{1}=\frac{\partial \mathbf{I}}{\partial \underline{\sigma}} \quad ; \quad \underline{\mathbf{b}}_{2}=\frac{\partial \bar{\sigma}}{\partial \underline{\sigma}}
$$




$$
\underline{\mathbf{b}}_{3}=\frac{\partial \theta}{\partial \bar{\sigma}} \underline{\mathbf{b}}_{2}+\frac{\partial \theta}{\partial \mathrm{J}_{3}} \frac{\mathrm{J}_{3}}{\partial \underline{\sigma}}
$$

where

$$
\begin{aligned}
& \frac{\partial \theta}{\partial \bar{\sigma}}=\frac{3 \sqrt{3} \mathrm{~J}_{3}}{2 \bar{\sigma}^{4} \cos 3 \theta} \\
& \frac{\partial \theta}{\partial \mathrm{J}_{3}}=-\frac{\sqrt{3}}{2 \bar{\sigma}^{3} \cos 3 \theta}
\end{aligned}
$$


APPENDIX B

\section{ISOTROPIC YIELD FUNCTIONS FOR THE ORIGINAL CRITICAL STATE CONCEPT}

The numerical simulation presented in Figure 4.5 were completed assuming the following functional forms of the yield criteria:

Cam-Clay model

$$
\mathrm{f}=\sqrt{3} \bar{\sigma}+\mathrm{mg}(\theta) \mathrm{I}\left[\ln \left(\mathrm{I} / \mathrm{I}_{0}\right)-1\right]=0
$$

Modified Cam-Clay model

$$
f=(I-a)^{2}+\frac{3 \bar{\sigma}^{2}}{m^{2} g^{2}(\theta)}-a^{2}=0
$$

where I, $\bar{\sigma}$, and $\theta$ are the stress invariants defined in section 4.2 , and the function $\mathrm{g}(\theta)$ describes the deviatoric cross-section of the yield locus. In the equations (B.1) and (B.2) the following definitions apply 


$$
\mathrm{m}=\frac{6 \sin \Phi}{3-\sin \Phi} ; \mathrm{a}=\mathrm{a}_{0} \exp \left(\frac{\mathrm{e}_{0}^{\mathrm{p}}-\mathrm{e}^{\mathrm{p}}}{\lambda-\kappa}\right) ; \mathrm{e}^{\mathrm{p}}=\left(1+\mathrm{e}_{0}\right) \epsilon_{\mathrm{i} i}^{\mathrm{p}}
$$

where $\Phi$ is the angle of internal friction and $\lambda, \kappa$ are material constants identified from hydrostatic compression test. The results of numerical simulations presented in Figure 4.5 pertain to $\theta=\pi / 6$. 


\section{B I B L O G R A P H Y}

1. Amadei, B. and Goodman, R.E. "Constitutive relation for fractured rock masses", Proceedings of International Symposium on The Mechanical Behaviour of Saturated Media, Ottawa, 1981.

2. Anandarajah, A. and Dafalias, Y.F. "Bounding surface plasticity. III: application to anisotropic cohesive soils", Journal of Engineering Mechanics ASCE, vol.112, 1986, 1292-1318.

3. Arthur, J.R.F, Bekenstein, S., Germaine, J.T. and Ladd, C.G. "Stress path tests with controlled rotation of principal stress directions",in: R.N. Young and F.C. Townsend, eds., Laboratory Shear Strength of Soil, American Society for Testing Materials, Special Technical Publication no. 740, Philadelphia, Pa., 1981.

4. Arthur, J.R.F, Chua, K.S. and Dunstan, T. "Induced anisotropy in sands", Geotechnique, vol.27, 1977, 13-33.

5. Atkinson, J.H. and Bransby, P.L. The Mechanics of Soils; An Introduction to Critical State Soil Mechanics. McGraw-Hill, 1978.

6. Attewell, P.B. and Farmer, I.W. Principies of Engineering Geology. New York, Halsted Press, a Division of John Wiley \& Sons Inc., 1976.

7. Baltov, A. and Sawczuk, A. "A rule of anisotropic hardening", Acta Mechanica, vol.1, 1965, 81-92.

8. Barden, L. "The influence of structure on deformation and failure in clay soil", Geotechnique, Vol.22, 1972, 159-163.

9. Bazant, Z.P. "Microplane model for strain-controlled inelastic behaviour", in: Mechanics of Engineering Materials, C.S. Desai and R.H. Gallagher, eds., New York, John Wiley \& Sons, 1984, 45-59.

10. Blyth, F.G.H and de Freitas, M.H. A Geology for Engineers. London, Butler \& Tanner Ltd., 1984. 
11. Boehler, J.P. "On a rational formulation of isotropic and anisotropic hardening" in: Applications of Tensor Functions in Solid Mechanics J.P Boehler ,ed., CISM Courses and Lectures no.292, Udine, Springer-Verlag, 1987, 99-122.

12. Boehler, J.P and Sawczuk, A. "On yielding of oriented solids", Acta Mechanica, vol.27, 1977, 185-206.

13. Bowen, N.L. "The reaction principle in petrogenesis", Journal of Geology, vol.30, 1922, 177-198.

14. Carter, J.P., Booker, J.R. and Worth, C.P. "A critical state model for cyclic loading in soil mechanics" in: Soil Mechanics-Transient and Cyclic Loads, G.N. Pande and O.C. Zienkiewicz, eds., John Wiley \& Sons, 1982, 219-252.

15. Casagrande, A. "The structure of clay and its importance in foundation engineering" Journal of the Boston Society of Civil Engineers, vol.19, 1932, 168-209.

16. Chalmers, B. The Structure and Properties of Solids. London, Heyden \& Son Ltd, 1982.

17. Chen, W.F. and Baladi, G.Y. Soil Plasticity, Theory and Implementation. Amsterdam, Elsevier, 1985.

18. Cormeau, I. "Numerical stability in quasi-static elasto/viscoplasticity", International Journal for Numerical Methods in Engineering, vol. 9, 1975, 109-127.

19. Cowin, S.C. "Fabric dependence of an anisotropic strength criterion", Mechanics of Materials, vol.5, 1986, 251-260.

20. Cowin, S.C. "The relationship between the elasticity tensor and the fabric tensor", Mechanics of Materials, Vol.4, 1985, 137-147.

21. DeBorst, R. and Vermeer, P.A. "Possibilities and limitations of finite elements for limit analysis", Geotechniqe, vol. 34, 1984, 199-210.

24. Defalias, Y.F. "Bounding surface plasticity; Part I: Mathematical foundations and hypoplasticity",Journal of Engineering Mechanics ASCE, vol.112, 1986, 966-987. 
22. Defalias, Y.F. and Herrmann, L.R. "Bounding surface formulation of soil plasticity" in: Soil Mechanics - Transient and Cyclic Loads, G.N. Pande and O.C. Zienkiewicz, eds., John Wiley \& Sons, 1982, 133-173.

23. Defalias, Y.F. and Popov, E.P. "A model of nonlinearly hardening materials for complex loadings", Acta Mechanica, vol. 21, 1971, 173-192.

25. Delage, P. and Lefebvre, G. "Study of the structure of a sensitive Champlain clay and of its evolution during consolidation", Canadian Geotechnical Journal, vol.21, 1984, 21-35.

26. Desai, C.S. "Thin-layer elements for interfaces and joints", International Journal For Numerical and Analytical Methods In Geomechanics, vol.8, 1985, 19-43.

27. Desai, C.S. and Gallagher, R.H. Mechanics of Engineering Materials. John Wiley \& Sons Ltd., 1984.

28. Desai, C.S. and Siriwardane, H.J. Constitutive Laws For Engineering Materials. Englewood Cliffs, New Jersay, PrenticeHall Inc., 1984.

29. Douglass, P.M. and Voight, B. "Anisotropy of granites: a refiection of microscopic fabric", Geotechnique, vol.19, 1969, 376398.

30. Duncan, J.M. and Seed, H.B. "Anisotropy and strength reorientation in clay", Journal of the Geotechnical Engineering Division Proceedings of the ASCE, vol.92, no.5, 1966, 21-50.

31. Feda, J. Mechanics of Particulate Materials. Prague, Elsevier Scientific Publishing Company, 1982.

32. Gelfand, I.M., Minlos, R.A. and Shapiro, Z.Ya. Representations of The Rotation and Lorentz Groups and Their Applications. New York, A Pergamon Press Book, The MacMillan Company, 1963.

33. Goodman, R.E. and Gen-hua, S. Block Theory and Its Applications to Rock Engineering. Englewood Cliffs, New Jersay, Prentice-Hall, 1985. 
34. Goodman, R.E., Taylor, R.L. and Brekke, S. "A model for the mechanics of jointed rocks", Soil Mechanics and Foundation, vol.94, 1968, 637-659.

35. Grabowska-Olszewska, B. and Siergiejew, J.M. Soil Physics. Warsaw, Wydawnictwo Geologiczne, 1977 (in Polish).

36. Haviicek, J. and Myslivec, A. "Influence of saturation and stratification on the shearing properties of certain soil", Proceedings of the 6th ICOSMFE, Montreal, vol.1, 1965, 235239.

37. Henkel, D.J. and Sowa, V.A. "The influence of stress history on stress path in undrained triaxial tests on clay", Proceedings of Conference on Laboratory Shear Testing of Soils, ASTM Special Technical Publications, no.361, 1963, 280-291.

38. Hill, R. The Mathematical Theory of Plasticity. London, Oxford University Press, 1950.

39. Hillard, J.E. "Measurement of volume in volume" in: Quantitative microscopy R.T DeHoff and F.N. Rhines, eds., New York, MicGraw-Hill, 1968, 45-76.

40. Hong, W. P. and Lade, P.V. "Elasto-plastic behaviour of Koconsolidated clay in torsion shear tests", Soils and Foundations, vol.29, no.2, 1989, 127-140.

41. Ingles, B.A. and Lee, I.K. "The influence of initial grain shape and pore anisotropy on strength of brittle soils", Geotechnique, vol.21, 1971, 143-153.

42. Jamiolkowski, M., Ladd, C.C., Germaine, J.T. and Lancellotta R. "New developments in field and laboratory testing of soils", Theme Lecture XI ICSMFE, San Francisco, 1985.

43. Jiang, J. and Pietruszczak, S. "Convexity of yield loci for pressure sensitive materials", Computer and Geotechnics, vol. 5, 1989, 5163.

44. Johnson, W., Sowerby, R. and Ventor, R.D. Plane Strain Slip Line Fields For Metal-Deformation Processes: A Source Book and Biblography. Oxford - Toronto, Pergamon Press, 1982. 
45. Kanatani, Ken-Ichi "Procedures for steroological estimation of structural anisotropy", International Journal Engineering Science, vol.23, no. 4, 1985, 587-598.

46. Kanatani, Ken-Ichi "Measurement of crack distribution in a rock mass from observations of its surface", Soils and Foundations, vol.25, 1985, 77-83.

47. Kanatani, Ken-Ichi "Distribution of directional data and fabric tensor", International Journal Engineering Science, vol.22, no.2, 1984, 149-164.

48. Kavazanjian, E.Jr. and Mitchell, J.K. "Time-dependent deformation behaviour of clays" Journal of the Geotechnical Engineering Division ASCE, vol.106, 1980, 611-630.

49. Khera, P. and Krizek, R.J. "Effect of principal consolidation stress difference on undrained shear strength", Soils and Foundations, vol.8, 1968, 1-17.

50. Kirkpatrick, W.M. and Rennie, I.A. "Directional properties of consolidated kaolin", Geotechnique, vol.22, 1972, 166-169.

51. Krieg, R.D. and Krieg, D.B. "Accuracies of numerical solution method for the elastic-perfectly plastic model", Journal of Pressure Vessel Technology, Transactions of the ASME, vol.99, 1977, 510-515.

52. Ladd, C.C. "Stress-strain behaviour of anisotropically consolidated clays during und ained shear", Proceedings of the 6th ICOSMFE, Montreal, vol.1, 1965, 282-286.

53. Lade, P.V. and Kim, M.K. "Yield criterion and plasticity work conturs for frictional materials", Computer and Geotechnics, vol.6, 1988, 13-31.

54. Lambe, T.W. "The engineering behaviour of compacted clay", Journal of the Geotechnical Engineerin: Division Proceedings of the ASCE, vol.84, 1958, 16-55.

55. Lee, K.L. and Morrison, R.A. "Strength of anisotropically consolidated compacted clay", Journal of the Geotechnical Engineering Division Proceedings of the ASCE, vol.96, no.6, 1970, 2025-2043. 
56. Lefebvre, G. and LeBoeuf, D. "Rate effects and cyclic loading of sensitive clays", Journal Geotechnical Engineering Division ASCE, vol.113, 1987, 476-489.

57. Lafeber, E. "Soil Structural Concepts", EngineeringGeology, vol.1, 1966, 261-290.

58. Lewin, P.I. and Burland, J.B. "Stress-probe experiments on saturated normally consolidated clay", Geotechnique, vol.20, 1970, 38-56.

59. Malvern, L.E. Introduction to The Mechanics of A Continuous Medium. Englewood Cliffs, New Jersey, Prentice-Hall Inc., 1969.

60. McConnachi, I. "Fabric changes in consolidated kaolin", Géotechique, vol.24, 1974, 207-222.

61. Mitchell, J.K. Fundamentals of Soil Behaviour. John Wiley \& Sons, Inc., 1976.

62. Mitchell, R.J. "Some deviations from isotropy in a lightly overconsolidated clay", Geotechnique, vol.22, 1972, 459-467.

63. Miura, K. Miura, S. and Shosuke T. "Deformation behaviour of anisotropic dense sand under principal axes rotation", Soils and Foundations, vol.26, 1986, 36-52.

64. Morgenstern, N.R. and Tchalenko, J.S. "Microscopic structures in kaolin subjected to direct shear", Geotechnique, vol.17, 1967, 309328.

65. Mroz, Z. "On isotropic hardening constitutive models in soil mechanics", International Workshop on Constitutive Relations for Soils, Grenoble, 6-8 September 1982, 463-469.

66. Mroz, Z. and Kwaszczynska, K. "Some boundary value problems for density hardening media", Rozprawy Inzynierskię, vol.19, 1971, $15-42$ (in Polish).

69. Murakami, S. "Anisotropic aspects of material damage and application of continuum damage mechanics" in: Continuum damage mechanics: theory and applications, D. Krajcinovic and J. Lemaitre, CISM Courses and Lectures no.295, Udine, SpringerVerlag, 1987, 91-134. 
68. Murakami, S. and Sawczuk, A. "On description of rateindependent behaviuur for prestrained solids", Archives of Mechanics, vol.32, 1979, 251-264.

69. Nayak, G.C. and Zienkiewicz, O.C. "Convenie!t form of stress invariants for plasticity", Journal of the Structural Division Proceedings of the ASCE, vol.98, 1972, 949-954.

70. Nayak, G.C. and Zienkiewicz, O.C. "Elasto-plastic stress analysis. A generalization for various constitutive relations including strain softening", International Journal for Numerical Methods in Engineering, vol. 5, 1972, 113-135,.

71. Naylor, D.J., Pande, G.N., Simpson, B. and Tabb, R. Finit Elements in Geotechnical Engineering. Swansea, Pineridge Press, U.K., 1981.

72. Ochiai, H. and Lade, P.V. "Three-dimensional behaviour of sand with anisotropic fabric" Journal of Geotechnical Engineering ASCE, vol.109, 1983, 1313-1328.

73. Oda, M. "Initial fabrics and their relations to mechanical properties of granular materials", Soils and Foundations, vol.12, $1972,17-36$.

74. Onat, E.T. and Leckie, F.A. "Representation of mechanical behaviour in the presence of changing internal structure", Journal of Applied Mechanics, vol. 55, 1988, 1-10.

75. Pande, G.N and Pietruszczak, P. "A rational interpretation of pore pressure parameters", submitted to Geotechnige, 1989.

76. Pande, G.N. and Sharma, K.G. "Multilaminate model of clays; a numerical evaluation of the influence of rotation of principal stress axes", International Journa For Numerical and Analytical Methods In Geomechanics, vol.7, 1983, 397-418.

77. Pande, G.N. and Sharma, K.G. "Joint//interface elements and associated numerical ill-conditioning", !nternational Journal For Numerical and Analytical Methods In Gieomechanics, vol.3, 1979, 293-300.

78. Pietruszczak, S. and Krucinski, S. "Description of anisotropic response of clays using a iensorial measure of structural disorder", accepted for publication in Mechanics of Materials, 1989. 
79. Pietruszczak, S. "A flow theory for soil: concept of multiple neutral loading surfaces", Computers and Geotechnics, vol.2, 1986, 185-203.

80. Pietruszczak, S. and Stoll, F.E. "Constitutive model for jointed and fissured material", in: Numerical Models in Geomechanics, G.N. Pande, Van Impe, M. Jackson \& Sons Publishing Company, 1986, 191-196.

81. Pietruszczak, S. and Mroz, Z. "On hardening anisotropy of Koconsolidated clays" International Journal For Numerical and Analytical Methods In Geomechanics, vol.7, 1983, 19-38.

82. Pietruszczak, S. and Mroz, Z. "FEM analysis of deformation of strain softening materials", International Journal for Numerical Methods in Engineering, vol.7, 1981, 327-334.

83. Podgorski, J. "General failure criterion for isotropic media", Journal of Engineering Mechanics ASCE, vol.111, 1985, 188-201.

84. Prager, W. "A new method of analyzing stresses and strains in work-hardening plastic solids", Journal of Applied Mechanics, vol.23, 1956, 493-496.

85. Prevost, J.H. "Plasticity theory for soils stress-strain behaviour", Journai of the Engineering Mechanics Division, Proceedings of the ASCE, Vol.104, 1978, 1177-1196.

86. Pul:ch, R. "Physico-chemical processes which affects soil structure and vice versa", Appendix to the Proceedings of the International Symposium on Soil Structure, Gothenburg, Sweden, 1973, 27-35.

87. Fusch, R. "Microstructural changes in soft quick clay at failure", Canadian Geotechnical Journal, vol.7, 1970, 1-7.

88. Roscoe, K.H. Schofield, M.A. and Wroth, M.A. "On the yielding of soils", Geotechnique, vol.9, 1958, 22-53.

89. Rowe, P.W. "The stress-dilatancy relation for static equilibrium of an assembly of particles in contact", Proceedings of the Royal Society, vol. A269, 1962, 500-527. 
90. Rowlands, R.E. "Strength (failure) theories and their experimental correlation", Failure Mechanics of Composite Handbook of Composites III, Sih G.C. and Skudra A.M., eds., New York, Elsevier Science Publisher, Ch.2, 1985.

91. Runesson, K. "Implicit integration of elastoplastic relations with reference to soils", International Journal For Numerical and Analytical Methods In Geomechanics, vol. 11, 1987, 315-21.

92. Saada, A.S. and Bianchini, G.F. "The strength of one dimensionally consolidated clays" Journal of the Geotechnical Engineering Division Proceedings of the ASCE, vol.101, 1975, 1151-1164.

93. Saada, A.S. and Bianchini, G.F. "The strength of onedimensionally consolidated clays; closure." Journal of the Geotechnical Engineering Proceedings of the ASCE, vol.103, no. GT6, 1977, $655-660$.

94. Saada, A.S. and Townsend, F.G. "State of the art: laboratory strength testing of soils",in: R.N. Young and F.C. Townsend, eds., Laboratory Shear Strength of Soil, American Society for Testing Materials, Special Technical Publication no. 740, Philadelphia, Pa., 1981.

95. Suery, M., Jalinier, J.M. and Raphanel, J. "Damage evolution and its influence on metal forming" in: A. Sawczuk and G. Bianchi, eds., Plasticity Today, London, Elsevier Applied Science Publisher, 1985, 553-568.

96. Schofield, A. and Wroth, P. Critical State Soil Mechanics. New York, McGraw-Hill, 1968.

97. Shrivastava, H.P., Mroz, Z. and Dubey, R.N. "Yield criterion and the hardening rule for plastic solid", Zeitschrift furer Angewandte Mathematik und Mechanik (ZAMM), vol. 53, 1973, 625-633.

98. Skempton, A.W and Sowa, V.A. "The behaviour of saturated clays during sampling and testing", Geotechnique, vol.13, 1963, 269290.

99. Snycier, M.D. and Bathe, KJ. "A solution procedure for thermoelastic-plastic and creep problems", Nuclear Engineering and Design, vol. 64, 49-80, 1981. 
100. Sowerby, R. Lecture Notes on Theory of Plasticity. McMaster University, Hamilton, 1988.

101. Stolle, D.E.F. and Higgins, J. "Viscoplasticity and plasticity numerical stability revisited", in: Numerical Models in Geomechanics NUMOG III, S.Pietruszczak and G.N. Pande , eds., Barking, Essex, Elsevier Science Publishers Ltd., 1989, 431438.

102. Symes, M.J., Gens, A. and Hight, D.W. "Undrained anisotropy and principal stress rotation in saturated sand", Geotechnique, vol.34, 1984, 11-27.

103. Vakulenko, A.A. and Kachanow, L.A. "Continuum theory of cracked media", Mech. Tverdogo Tiela, vol.4, 1971, 159-166, (in Russian).

104. Varadarajan, A. and Pande, G.N. "Prediction of anisotropic behaviour of clays by the multilaminate model", submitted to Computer and Geotechnics, 1989.

105. Wong, R.K.S. and Arthur, J.R.F "Induced and inherent anisotropy in sand" Geotechnique, vol.35, 1985, 471-481.

106. Ziegler, H. "A modification to Prager's hardening rule", Quarterly Journal of Applied Mathematic, vol.17, 1959, 55-65.

107. Zienkiewicz, O.C and Pande, G.N. "Some useful forms of isotropic yield surfaces for soil and rock mechanics", in: Finite Elements in Geomechanics, Gudehus G., ed., Bristol, John Wiley and Sons, 1977, 179-190.

108. Zienkiewicz, O.C. and Pande, G.N. "Time dependent multilaminate model of rocks - a numerical study of deformation and failure of rock masses", International Journal For Numerical and Analytical Methods In Geomechanics, vol.1, 1977, 219-247.

109. Zienkiewicz, O.C. and Cormeau, I.C. "Visco-plasticity-plasticity and creep in elastic solids - a unified numerical solution approach", International Journal for Numerical Methods in Engineering, vol. 8, 1974, 821-845. 